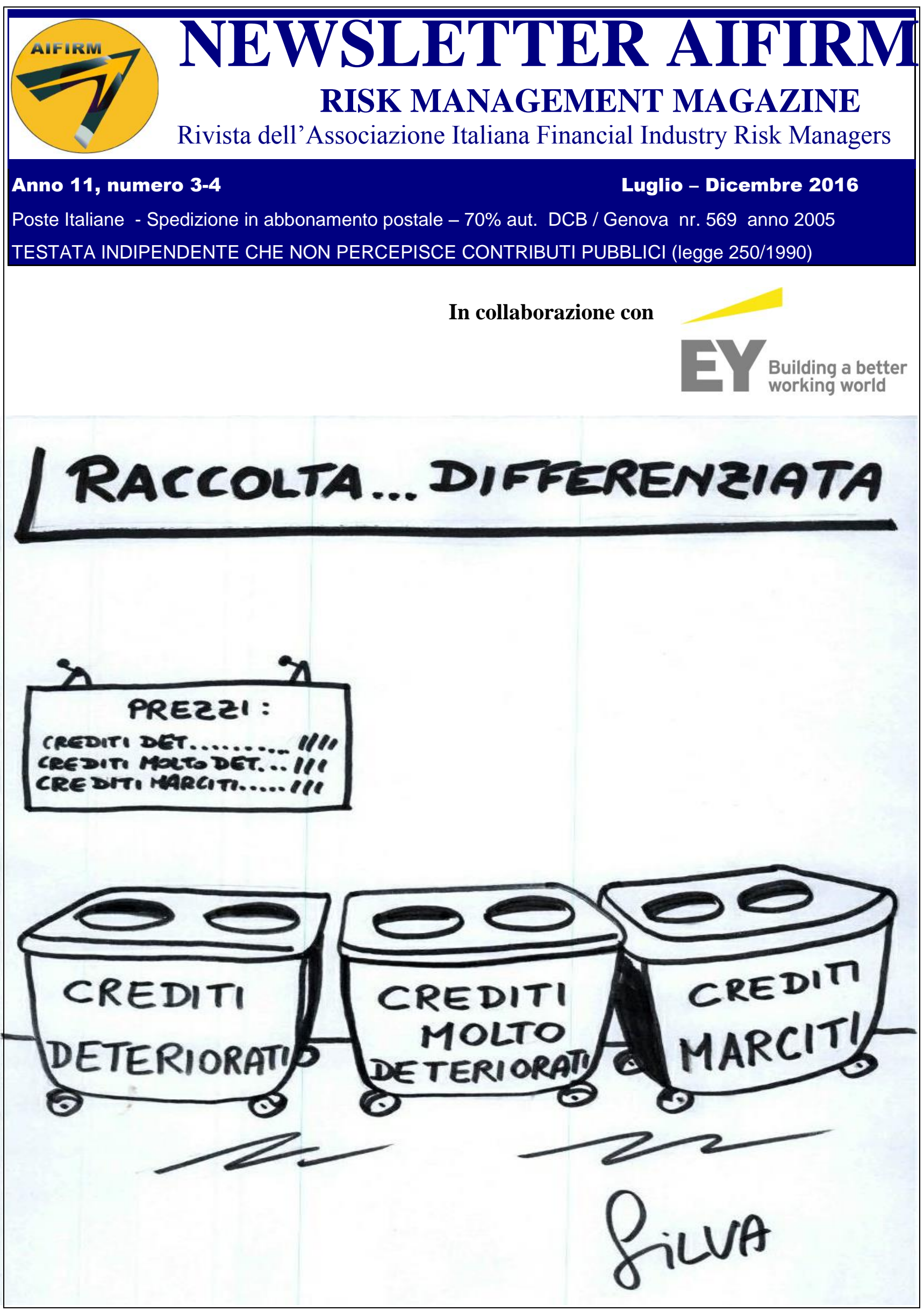

NEWSLETTER AIFIRM RISK MANAGEMENT MAGAZINE ANNO 11 No 3-4 - PAGINA - 1 - 


\section{IN QUESTO NUMERO}

\begin{tabular}{|c|c|}
\hline 3 & $\begin{array}{l}\text { Editoriale } \\
\text { di Maurizio Vallino }\end{array}$ \\
\hline 4 & $\begin{array}{l}\text { Gli scenari multipli IFRS9. Uno studio } \\
\text { applicativo } \\
\text { Daniele Monzali }\end{array}$ \\
\hline 14 & $\begin{array}{l}\text { L'esposizione al rischio di tasso di } \\
\text { interesse del portafoglio bancario: quali } \\
\text { implicazioni per le strategie di Asset \& } \\
\text { Liability Management? } \\
\text { Igor Gianfrancesco }\end{array}$ \\
\hline 41 & $\begin{array}{l}\text { Implementazione della Fuzzy Logic per la } \\
\text { gestione ottimale del portafoglio: la } \\
\text { modellizzazione dell'avversione al rischio } \\
\text { di un investitore attraverso tecniche di soft- } \\
\text { computing } \\
\text { Pier Giuseppe Giribone, Ottavio Caligaris, } \\
\text { Simone Fioribello, Simone Ligato }\end{array}$ \\
\hline
\end{tabular}

Processo di referaggio degli articoli proposti per la pubblicazione

Gli articoli che sono proposti alla rivista per la pubblicazione sono sottoposti in forma anonima a due successivi livelli di referaggio.

Il primo livello di referaggio (di ammissibilità) viene effettuato sull'articolo dai membri del Consiglio Scientifico che ne valutano la conguità ai temi trattati dalla rivista.

Il secondo livello di referaggio (di pubblicabilità) viene effettuato sull'articolo da due referee scelti all'interno del Consiglio Scientifico o all'esterno tra accademici, ricercatori, esperti della materia, che ne valutano il contenuto e forma.
Newsletter AIFIRM - Risk Management Magazine Anno 11, n 3-4 Luglio - Dicembre 2016

Direttore Responsabile:

Maurizio Vallino

Condirettore

Corrado Meglio

Comitato scientifico

Simona Cosma

Paola Ferretti

Giampaolo Gabbi

Andrea Giacomelli

Pier Giuseppe Giribone

Cristiana Schena

Giuseppe Torluccio

Enzo Scannella

Comitato di redazione: Emanuele Diquattro, Fausto Galmarini, Rossano Giuppa, Aldo Letizia, Paolo Palliola, Enzo Rocca, Fabio Salis

\section{Vignettista: Silvano Gaggero}

Proprietà, Redazione e Segreteria:

Associazione Italiana Financial Industry Risk Managers (AIFIRM), Via Sile 18, 20139 Milano

Registrazione del Tribunale di Milano $n^{\circ} 629$ del $10 / 9 / 2004$

\section{ISSN 2283-7329}

E-mail: segreteria@aifirm.it;

Tel. 3896946315

Lunedì h.10-12; da Lunedì a Venerdì h.15-17

Stampa: Algraphy S.n.c. - Passo Ponte Carrega 62-62r 16141 Genova

Le opinioni espresse negli articoli impegnano unicamente la responsabilità dei rispettivi autori

SPEDIZIONE IN ABBONAMENTO POSTALE AI SOCI AIFIRM RESIDENTI IN ITALIA, IN REGOLA CON L'ISCRIZIONE

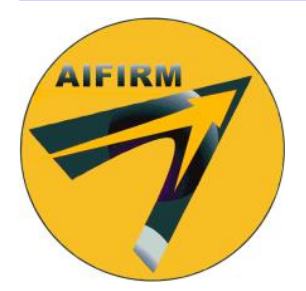




\section{EDITORIALE}

Cari lettori,

la XII Convention Aifirm affronta il tema dell'aleatorietà dei modelli di risk management in una congiuntura in cui la scelta dell'ampiezza delle serie storiche può determinare misurazioni di rischio anche molto diverse e, conseguentemente, risultati di pricing molto differenti.

Ciò vale in particolare nel mondo del credito dove l'applicazione dei modelli di risk management, che utilizzano i fenomeni di volatilità del passato per misurare il livello di capitale da accantonare, riporta risultati fortemente dipendenti dalle scelte di modello (ampiezza delle serie storiche e fattori $\lambda$ utilizzati) in un ambiente finanziario caratterizzato da scossoni che lo rendono sempre più volatile e imprevedibile.

Tale spunto vale altresì nel mercato, al momento rarefatto ma in potenza emergente, della negoziazione degli NPL.

L'applicazione su tali asset dei modelli di risk management dovrebbe includere variabili che, in quanto in buona parte qualitative, risultano difficilmente modellizzabili pur se fortemente determinanti per un corretto pricing.

Prendiamo ad esempio le sofferenze assistite da garanzia immobiliare.

L'analisi classica dovrebbe condurci ad un valore dell'asset pari a EAD*(1-LGD) con una LGD calcolata su una serie storica di circa 10 anni.

Tuttavia tra le variabili "ambientali" che potrebbero portare ad uno scostamento del valore delle NPL in caso di cessione, rispetto ad una modelizzazione "classica", possiamo considerare almeno le seguenti:

- il forte rigore imposto dall'Organismo di Vigilanza nelle svaluzioni degli NPL;

- la forte volatilità del mercato immobiliare che ha visto un calo molto marcato negli ultimi anni ma che potrebbe mostrare una ripresa nel futuro;

- la velocizzazione dei tempi di vendita degli immobili all'asta dovuta, in molte regioni, ad un'accelerazione dei tempi dei tribunali e ad una migliore pubblicità delle aste attraverso internet;

- la mappatura del numero di aste già bandite per gli immobili a garanzia. A tale proposito è di interesse il fenomeno degli immobili già aggiudicati in asta e pagati dall'aggiudicatario ma il cui credito di riferimento risulta ancora in NPL nelle more di chiusura della pratica da parte del tribunale.

Il mutamento, sia pure lento, del quadro finanziario ed economico fa ritenere che le cessioni di NPL siano un migliore affare per i prenditori che per i venditori in quanto molto valore potrebbe essere nascosto dietro il velo dell'elevata prudenzialità delle valutazioni.

Sarà solo il tempo a dirci se, insieme agli NPL, uscirà dai bilanci delle banche del valore che sarebbe spettato agli azionisti.

Maurizio Vallino 


\section{Gli scenari multipli IFRS9. Uno studio applicativo}

di Emilio Maffi (EY), Daniele Monzali (EY) e Giuseppe Quaglia (EY)

\section{Abstract.}

The introduction of the new IFRS9 Accounting Principle for the computation of loan loss provisions prescribes a shift to a new loan classification scheme based on three classes (Stage) and to the calculation of provision coverages according to an expected loss philosophy. This paper is aimed at analysing specific IFRS9 requirements and ITG technical requirements for the forward looking PIT calibration of expected loss measures accounting for multiple scenarios and at presenting a set of simulations of tests, measures and IFRS9 allowance and coverage calculation in a multiple scenario regime. The key result is an empirical assessment of the sensitivity of IFRS9 provisions to the probability assigned to adverse scenarios, where it is shown that under certain circumstances the impact of the so-called "non linearity" on IFRS9 provisions may be non-negligible.

Requisiti e guidelines Regolamentari.

Il Principio Contabile IFRS 9 redatto dallo IASB prevede l'introduzione di un unico modello di impairment valido per tutti gli strumenti finanziari non rilevati al Fair Value con contropartita a conto economico. Il modello proposto è basato su un concetto di perdita attesa (Expected Credit Loss) che sia il risultato unbiased (i.e. che non sia né ottimistica né prudenziale [1]) della ponderazione di tutti i possibili scenari attesi [2] e che includa la valutazione di informazioni forward looking [3] disponibili senza undue cost or effort [4]. Secondo il nuovo Principio, le informazioni forward looking e gli scenari macroeconomici devono essere presi in considerazione sia per il calcolo delle Expected Credit Losses (ECL) [2], al fine di garantire una misura il più possibile unbiased, sia per la valutazione del significativo deterioramento del credito che rileva a fini di Stage Assignment [5].

Il forum di discussione ITG (Transition Resource Group for impairment of Financial Instruments), istituito dallo IASB per fornire supporto implementativo, ha messo in discussione il principio generale di adottare un singolo scenario per il calcolo della ECL. Dalla discussione è emerso come tale possibilità non risulti coerente con il nuovo Principio in caso di relazione non lineare tra i diversi scenari forward looking e le ECL associate [6], poiché potrebbe violare il requisito di misura unbiased prescritta dal Principio [7]. Analogamente anche ai fini della stage allocation, quando si riscontra una relazione non lineare tra gli scenari forward looking e le relative perdite su crediti l'utilizzo di uno scenario singolo potrebbe non consentire di rispettare le finalità del Principio [8]. L'ITG ha sottolineato il requisito di coerenza (ove rilevante) fra le informazioni forward looking adottate per la misurazione dell'ECL, quelle utilizzate per la valutazione del significativo deterioramento del rischio creditizio [9] e quelle utilizzate per le misure di perdita attesa utilizzate nell'ambito di altri processi interni (e.g. budget e capital plan) [10]. Sebbene l'IFRS 9 non prescriva alcun metodo specifico per il calcolo dell'ECL e per lo stage assignment, le discussioni in seno all'ITG hanno condotto a oggi alla definizione di quattro possibili approcci [11] per considerare le informazioni forward looking a fini di impairment, che vengono approfonditi nella sezione successiva di questo documento.

I requisiti definiti dallo IASB e approfonditi a fini implementativi dall'ITG sono stati recepiti dal Comitato di Basilea (BCBS) e dall'EBA, che hanno pubblicato le rispettive Guidelines nel Dicembre $2015^{1}$ e nell'Ottobre $2016^{2}$. I due organi hanno ribadito come per la stima della ECL sia essenziale che ogni ente creditizio prenda in considerazione tutte le informazioni forward looking ragionevoli e sostenibili, inclusi i fattori macroeconomici [12], in quanto tali informazioni rappresentano un fattore critico per poter rilevare le rettifiche di valore su crediti in modo accurato e tempestivo $^{3}$ [13] . Il Comitato di Basilea ha in questo senso rafforzato la richiesta di utilizzare le informazioni forward looking ai fini della definizione della ECL, limitando il ricorso alla giustificazione di undue cost or effort per evitarne o ridurne gli impatti sull'implementazione [14].

A livello di industry l'Institute of International Finance (IIF), che ha come obiettivo il supporto delle società finanziarie ai fini di una sana gestione dei rischi (sotto molteplici profili incluso quello contabile), ha declinato dal punto di vista di workflow di calcolo alcuni potenziali approcci di stage allocation e metodi di calcolo delle ECL discussi in seno all'ITG e ha evidenziato i punti di attenzione e le problematiche emergenti a livello internazionale nel cercare di declinare operativamente il Principio e le linee guida degli Organi di Regolamentazione (BCBS, EBA). Alla luce dell'esigenza di pervenire a risultati significativi e di rappresentare in maniera chiara l'evoluzione delle rettifiche di valore [15], l'IIF ritiene che si debba trovare un equilibrio tra la complessità del modello e la sua trasparenza. In questo contesto, si è da un lato espresso concorde con la posizione dell'ITG nell'utilizzo di un singolo scenario in presenza di una relazione lineare tra i diversi scenari forward looking e le ECL mentre dall'altro, in caso di un grado limitato di non linearità, ha previsto la possibilità di adottare un singolo scenario che consideri le molteplici possibili realizzazioni [16].

\footnotetext{
${ }^{1}$ Versione definitiva.

${ }^{2}$ Draft di consultazione.

${ }^{3}$ L'introduzione del nuovo standard IFRS9 è stata richiesta agli Standard Setters nel 2009 con l'obiettivo di superare la percepita inadeguatezza dello standard IAS39 corrente in termini di tempestività e adeguatezza del framework di provisioning ("Too little, too late").
} 
Infine, nel mese di Giugno 2016 anche il settore della Revisione Contabile si è espresso per promuovere una adeguata implementazione del nuovo Standard IFRS9 per tramite del Global Public Policy Committee (GPPC), che raccoglie rappresentanti delle sei maggiori società di revisione. A tal fine il GPPC ha pubblicato il documento "The implementation of IFRS 9 impairment requirements by banks", in cui si promuove una implementazione di alto livello dei processi contabili per la rilevazione delle ECL e vengono codificati elementi di principio e operativi a supporto degli auditors nelle valutazioni da effettuare durante le fasi di realizzazione e transizione al nuovo Standard. A tal proposito, il documento propone due modelli alternativi per la misurazione delle ECL coerenti con gli approcci definiti dal gruppo ITG [11]. Il GPPC prevede infatti la possibilità di calcolo delle ECL mediante un singolo scenario a cui devono essere sommate o sottratte una eventuale rettifica, modellizzata separatamente, volta a riflettere gli effetti di altri scenari meno probabili con i connessi impatti non lineari [17], ed una rettifica ulteriore concepita per comprendere i c.d. additional factors che esprimono l'effetto di eventi idiosincratici non esplicitamente incorporati nella modellistica delle ECL. E' importante sottolineare che sebbene non inclusi nella modellistica ECL, tali eventi sono nondimeno considerati possibili e potrebbero avere un effetto significativo sulle perdite attese [18]. Il GPPC ha inoltre esplicitamente evidenziato come l'utilizzo di un singolo scenario in presenza di effetti di non linearità [19] e l'utilizzo di previsioni che siano esclusivamente sviluppate internamente o che si riferiscano ad una sola fonte esterna [20] potrebbero non essere compliant con il Principio.

Opzioni implementative.

Le risultanze dell'ultimo meeting dell'ITG e il rilascio delle guidance regolamentari e di Audit da parte rispettivamente dell'EBA e del GPPC hanno aperto una riflessione nel settore bancario sulle possibili modalità di implementazione dei requisiti relativi alla gestione di non linearità e scenari multipli a fini IFRS9.

L'ITG paper presenta 4 possibili modalità di gestione degli scenari a fini di calcolo delle ECL:

- (1) utilizzo del singolo scenario più probabile (single most-likely scenario) e calcolo delle ECL ad esso condizionate;

- (2) utilizzo di un singolo scenario costruito ponderando i possibili scenari per le relative probabilità di realizzazione (single averaged scenario) e calcolo delle ECL ad esso condizionate;

- (3) calcolo delle LECL condizionate ad ogni possibile scenario e ponderazione ex-post delle ECL condizionate con le probabilità di realizzazione degli scenari utilizzati (multiple scenarios);

- (4) utilizzo del singolo scenario più probabile (overlay to single most-likely scenario), calcolo della ECL ad esso condizionata e aggiustamento di quest'ultima con un overlay rappresentativo degli scenari meno probabili (le cosiddette minority views).

L'opzione (3) viene considerata da ITG l'opzione più in linea con lo standard, in quanto riflette pienamente l'obiettivo di calcolare la perdita attesa come "an unbiased and probability-weighted amount that is determined by evaluating a range of possible outcomes".

L'opzione (1) viene considerata da ITG coerente con il nuovo standard solo in alcune situazioni, i.e. quelle in cui il singolo scenario adottato sia rappresentativo di una gamma di possibili scenari in termini di perdita attesa. Da un punto di vista operativo, per esempio, situazioni di questo tipo sono quelle in cui non siano presenti fenomeni di c.d. non linearità fra scenari e perdita attesa, vale a dire nei casi in cui non vi siano differenze materiali rispetto alla LECL calcolata secondo l'opzione (3).

L'opzione (4) costituisce un c.d. practical expedient che può essere considerato coerente con lo Standard e più snello a fini di implementazione del calcolo della perdita attesa nei sistemi IT, in quanto non richiede il calcolo condizionato a diversi scenari a ogni reporting date. A tal fine l'overlay da applicare al calcolo basato sul singolo scenario più probabile deve essere determinato in coerenza con gli obiettivi dello Standard e le prescrizioni dell'ITG, e quindi in particolare riflettere pienamente l'eventuale non linearità riscontrata senza considerare in sovrapposizione scenari macroeconomici ed eventi considerati probabili anche se di natura idiosincratica [21].

E' quindi possibile ipotizzare alcune linee guida operative per la definizione del processo di calcolo delle perdite attese secondo il nuovo standard:

- Processo di calcolo delle perdite attese:

○ Il processo di calcolo delle perdite attese potrà essere implementato con criteri aderenti a dimensione/complessità dell'intermediario, caratteristiche dei portafogli e disponibilità dei dati, senza prescrizioni specifiche per una particolare metodologia, ferma restando la coerenza con gli obiettivi definiti dal principio;

○ In generale, occorre considerare di più di uno scenario ai fini della misurazione della perdita attesa ma non è sempre necessario utilizzare scenari multipli per il calcolo degli accantonamenti di bilancio;

○ Appare quindi sempre necessario per gli intermediari effettuare un test di non linearità; 
$\circ$ Nelle situazioni in cui si evidenzi una relazione non lineare fra scenari forward-looking e perdite attese il rispetto delle finalità del principio richiede l'utilizzo di scenari multipli per la determinazione delle perdite attese a fini di accantonamento (opzioni (3) o (4) come sopra).

\section{- Scelta e probabilizzazione degli scenari:}

- Non è necessario considerare ogni possibile scenario ma gli scenari da prendere in considerazione dovrebbero rappresentare comunque un campione rappresentativo della gamma dei possibili scenari;

- La gamma di scenari considerati e le relative probabilità di accadimento devono riflettere le attese dell'intermediario, anche nel caso in cui vengono utilizzati dati e/o scenari esterni a supporto del framework implementativo;

- Sarebbe necessario che l'intermediario consideri sia scenari esterni, ad esempio scenari "Consensus" e/o scenari regolamentari, che scenari interni, ad esempio sottesi a Piani Strategici, Pianificazione, Budget e Capital Plan, a supporto della propria "own view" di scenario;

- Non sono in linea di principio opzioni compliant con lo standard:

- l'utilizzo di un singolo scenario favorevole o prudenziale, dato che occorre definire una stima "non distorta" delle perdite attese ("unbiased... amount");

- l'utilizzo di un insieme di scenari "central"/"baseline" alternativi, che non costituisce un campione rappresentativo degli scenari possibili.

- Impatti sulla procedura di Staging allocation: la procedura di staging deve essere coerente con il processo di calcolo delle perdite attese in relazione alla considerazione di informazioni forward-looking. In particolare, $\mathrm{i}$ test di non linearità dovrebbero consentire di individuare se i driver di eventuale non linearità fra scenari e stime di perdita sono rilevanti anche a fini di rilevazione del significativo incremento del merito creditizio e, in caso affermativo, dovrebbero essere considerati a fini di staging allocation.

\section{Una simulazione di impatto}

I requisiti previsti dallo Standard IFRS9 e le risultanze dell'ultimo meeting dell'ITG sono stati declinati in questo paper mediante l'opzione implementativa multiple scenarios definita al punto (3) della sezione precedente, che prevede la ponderazione ex-post delle LECL condizionate ad ogni possibile scenario con le probabilità di realizzazione degli scenari utilizzati. L'obiettivo è quello di presentare i risultati di due simulazioni basate sull'utilizzo di scenari multipli, verificarne la linearità dell'impatto [22] e fornire un'analisi di sensitivity sulla base delle probabilità di realizzo degli scenari.

In coerenza con il Principio e in linea con il dibattito emerso nel contesto internazionale, la LECL è calcolata, per ogni scenario considerato, come somma delle expected losses definite sulla base dell'insieme dei parametri previsionali associati ai timesteps $\mathrm{t}$ da data reporting lungo la durata residua attesa del contratto, $\mathrm{T}$ :

$$
L E C L_{T}=\sum_{t=1}^{T}\left(1-C P D_{t-1}\right) * P D_{t} * E A D_{t} * D F_{t} * L G D_{t}
$$

$C P D_{t-1}:$ probabilità di default cumulata fino a $t-1$;

$P D_{t}: \quad$ probabilità di default marginale in $t$;

$E A D_{t}: \quad$ esposizione a default in $t$;

$D F_{t}: \quad$ discount factor in $t$;

$L G D_{t}$ : Loss Given Default in $t$.

Gli scenari

Le due simulazioni si basano sulla definizione di due set di scenari multipli a fini di studio di impatto, strutturati e probabilizzati sull'orizzonte 2016-2018 come segue:

- Scenari multipli Oxford Economics [23], che definisce delle probabilità di realizzazione per 6 possibili scenari, fra i quali uno scenario è baseline, quattro scenari sono adverse/di downturn (China hard landing, Financial market contagion, Geopolitical tensions, Weak commodity demand) e uno scenario postula un clima macroeconomico complessivo più favorevole rispetto allo scenario baseline (World growth surges);

- Scenari multipli Stress Test EBA 2016 [24]/Oxford Economics: i due scenari specificati dall'EBA per i Supervisory Stress Tests 2016 (Baseline scenario e Adverse scenario di downturn) sono stati integrati con un terzo scenario favorevole "World growth surges" mututato da Oxford Economics; ai tre scenari sono state attribuite delle probabilità di realizzazione su base judgemental. 
A fini di calcolo delle metriche IFRS9 è necessario che negli scenari considerati siano valorizzate tutte le variabili risultate significative nei modelli previsivi per tassi di default/PD e LGD. Nello studio presentato si è reso necessario definire per gli scenari Oxford Economics alcune ipotesi di valorizzazione relativamente ad alcune variabili mancanti:

- Swap Rates: i tassi swap sono presenti solo per lo scenario Baseline e sono stati approssimati per i restanti scenari per interpolazione fra i tassi dei Titoli di Stato con rating BB e la curva Swap dello scenario Baseline;

- Residential Property Price (RPP): anche 1'RPP è valorizzato solo nello scenario Baseline ed è stato quindi stimato per i restanti scenari mediante interpolazione del Consumer Price Index (CPI) con House Price Index (HPI);

- Commercial Property Price (CPP): il CPP non è valorizzato in nessuno degli scenari ed è stato quindi approssimato a partire dai valori di RPP, ai quali è stato sommato il differenziale fra CPP e RPP valorizzati negli scenari EBA.

La trasmissione degli scenari ai parametri di rischio sull'orizzonte lifetime necessario per una simulazione IFRS9 è stata strutturata in una componente di breve/medio periodo, coperta dai valori puntuali di scenario, e in una componente di lungo periodo.

Per gli anni compresi nell'orizzonte temporale 2016-2018 le previsioni di PD e LGD sono stimate condizionatamente ai valori delle variabili macroeconomiche e finanziarie specificati negli scenari. I valori di lungo periodo sono poi stati fissati secondo una logica di mean reversion, i.e. la PD di long run è stata calibrata riproporzionando i tassi di decadimento medi long run benchmark di Banca d'Italia [25] in relazione ai default rate del portafoglio di riferimento mentre la LGD di long run è stata stimata assumendo la stabilità dei prezzi degli immobili commerciali e residenziali successivamente al 2018.

Il portafoglio di simulazione e i relativi parametri di rischio e di attualizzazione

Le simulazioni sono state condotte su un portafoglio benchmark al 31/12/2015 costruito secondo criteri di segmento, prodotto, maturity e collateralizzazione rappresentativi di una banca commerciale di medie dimensioni, del quale si riporta il dettaglio in tabella 1.

\begin{tabular}{|l|c|c|c|}
\hline \multicolumn{1}{|c|}{ Segmento } & Esposizione \% & LGD Media & PD Media \\
\hline Corporate & $\mathbf{4 6 . 0 3 \%}$ & $\mathbf{3 4 . 8 9 \%}$ & $\mathbf{5 . 9 2 \%}$ \\
\hline di cui: Bullet & $9.19 \%$ & $45.00 \%$ & $5.92 \%$ \\
\hline di cui: Current Account & $12.83 \%$ & $45.00 \%$ & $5.92 \%$ \\
\hline di cui: Loan & $24.02 \%$ & $25.62 \%$ & $5.92 \%$ \\
\hline Retail & $\mathbf{3 6 . 3 8 \%}$ & $\mathbf{2 4 . 4 5 \%}$ & $\mathbf{1 . 9 2 \%}$ \\
\hline di cui: Consumer Loans & $7.44 \%$ & $65.00 \%$ & $1.92 \%$ \\
\hline di cui: Loan & $\mathbf{2 8 . 9 4 \%}$ & $14.04 \%$ & $1.92 \%$ \\
\hline Small Business & $\mathbf{1 7 . 5 9 \%}$ & $\mathbf{3 1 . 2 8 \%}$ & $\mathbf{6 . 5 8 \%}$ \\
\hline di cui: Bullet & $3.51 \%$ & $45.00 \%$ & $6.58 \%$ \\
\hline di cui: Current Account & $4.90 \%$ & $45.00 \%$ & $6.58 \%$ \\
\hline di cui: Loan & $9.17 \%$ & $18.70 \%$ & $6.58 \%$ \\
\hline Grand Total & $100.00 \%$ & $30.46 \%$ & $4.58 \%$ \\
\hline
\end{tabular}

Tabella 1 - Portafoglio di simulazione per tipologia di prodotto e scadenza (Si ipotizza una quota di defaulted assets pari al 10\% per ciascun sottosegmento Mln)

La PD è stata associata ai segmenti in relazione a scale di rating benchmark per segmento in 5 classi mentre la LGD è stata associata in base a una relazione benchmark proxy fra LGD e Value to Loan (VTL) delle garanzie. I valori risultanti di VTL sono poi stati raggruppati in 11 classi alle quali corrispondono diversi valori di LGD, distintamente per il segmento retail e per quello corporate.

L'EAD è stimata per ogni scadenza fino a maturity secondo una ipotesi semplificata di ammortamento lineare mentre per i prodotti a revoca è stata ipotizzata in via preliminare una scadenza di un anno.

Infine, Il discount factor $\left(\mathrm{DF}_{\mathrm{t}}\right)$ è stato determinato a partire dai valori benchmark dei tassi di interesse osservati sui segmenti Retail, SME e Corporate pubblicati da Banca d'Italia [26].

Data la natura benchmark del portafoglio al fine di condurre una simulazione realistica è stato necessario adottare ipotesi semplificate di staging allocation: è stato adottato a questo fine un criterio di staging basato su soglie assolute per il significativo deterioramento del rischio di credito della controparte alla reporting date: le esposizioni allocate in Stage 2, per le quali è prevista una misura lifetime di ECL, sono quelle a cui è associato un rating nelle ultime due classi performing della scala benchmark mentre, viceversa, sono state allocate in Stage 1, con associata una ECL a 1 anno, le 
esposizioni con associato un rating nelle prime tre classi della scala stessa. La quota risultante del portafoglio performing in Stage 2 è di circa il $28 \%$.

I modelli previsionali

La stima delle PD lifetime è stata effettuata con approccio Markoviano Non Omogeneo a partire da matrici di transizione benchmark e da un modello di previsione per le PD di portafoglio. La struttura a scadenza delle PD è cioè ottenuta attraverso la proiezione Markoviana di matrici di transizione a un anno costruite in coerenza con le ipotesi di scenario, vale a dire forward-looking per gli anni 2016 - 2018 e poi di lungo periodo.

A fini di previsione per ogni singolo scenario sono state calcolate le PD point-in-time di segmento riferite agli anni 2016-2017-2018, mediante un modello satellite PD che esprime la relazione storica tra i tassi di default forniti da Banca d'Italia [25] e le variabili macroeconomico-finanziarie. Le PD relative all'orizzonte previsionale 2016-2018 sono differenziate per segmento e sono stimate a partire dai valori scenario e dai coefficienti stimati dal modello secondo una logica di moltiplicatore, che consente di rendere coerenti le previsioni con i tassi di default del portafoglio. Il modello è stato stimato sull'arco temporale 1999Q1 - 2015Q4.

La tabella 2 riporta le variabili del modello satellite e il loro segno in termini di proporzionalità diretta (+) o inversa (-) con le PD.

\begin{tabular}{||l|l|l|}
\hline \multicolumn{1}{|c|}{ Corporate } & \multicolumn{1}{|c|}{ Small Business } & \multicolumn{1}{c|}{ Retail } \\
\hline Real GDP growth rate euro area (-) & Real GDP growth rate euro area (-) & House price index growth rate (-) \\
Consumer price growth rate Italy (-) & Consumer price growth rate Italy (-) & Consumer price growth rate Italy (-) \\
Unemployment rate Italy (+) & Unemployment rate Italy (+) & Unemployment rate Italy (+) \\
Btp 10 years return (+) & Swap rate 10 year (-) & $\begin{array}{l}\text { Swap rate 10 year }(-) \\
\text { Btp 10 years return (+) }\end{array}$ \\
\hline
\end{tabular}

Tabella 2 - Variabili macroeconomiche del modello satellite per le PD.

Per ottenere le matrici forward looking per i tre anni dell'orizzonte di previsione le matrice di transizione benchmark sono state ricalibrate sulle PD PIT determinate in base agli scenari e ai modelli satellite PD. In particolare, il processo di ricalibrazione della matrice avviene traslando le distribuzioni per classe mediante l'ottimizzazione della PD media della matrice rispetto all'Anchor point costituito dalle previsioni di PD stimate con il modello satellite, al fine di ottenere le matrici forward looking differenziate per anno e segmento. Questo approccio di stima delle matrici forward looking, per quanto semplificato, permette ad esempio la riconciliazione delle metriche IFRS 9 con quelle utilizzate negli esercizi di stress test.

Infine, la matrice di transizione di lungo periodo, che è stata applicata oltre l'orizzonte temporale di tre anni, è stata stimata mediante la definizione di un Anchor Point di lungo periodo ottenuto a partire dai tassi di decadimento Banca d'Italia in coerenza con i tassi di default del portafoglio benchmark.

Il calcolo della LGD Lifetime invece è stato effettuato mediante la relazione benchmark fra LGD e LTV delle garanzie e la rivalorizzazione del LTV stesso per ogni anno di previsione condizionatamente allo scenario macroeconomico considerato. In particolare, le due componenti del LTV sono state così calcolate:

- il valore prospettico del collateral è stato ottenuto per il periodo 2016-2018 sulla base delle previsioni EBA/OE dei prezzi del mercato immobiliare residenziale e commerciale ed è stato ipotizzato costante per gli anni successivi;

- l'esposizione è derivata dall'evoluzione dell'EAD nel tempo e tiene quindi in considerazione della diminuzione del debito residuo nel corso del tempo.

Risultati

Lo studio è stato effettuato attraverso due simulazioni di calcolo delle ECL. Per ciascuna di esse e per ogni scenario, si riportano:

- $\quad$ la EL a 12 mesi, calcolata su tutto il portafoglio;

- la Lifetime EL, calcolata su tutto il portafoglio;

- la Portfolio EL, calcolata ponderando la ECL a 12 mesi e Lifetime per le quote di portafoglio rispettivamente in Stage 1 e Stage 2.

Inoltre, per ciascuna delle due simulazioni sono state calcolate le medie ponderate ("Weighted") delle tre metriche di ECL condizionate agli scenari con le probabilità assegnate agli scenari stessi. 
La prima simulazione è stata effettuata a partire dai sei scenari riportati da Oxford Economics, per i quali sono fornite le probabilità di realizzazione riportate in tabella 3 [23].

\begin{tabular}{|l|c|c|c|c|c|c|}
\hline Scenario & China hard landing & $\begin{array}{c}\text { Financial market } \\
\text { contagion }\end{array}$ & $\begin{array}{c}\text { Geopolitical } \\
\text { tensions }\end{array}$ & $\begin{array}{c}\text { Weak commodity } \\
\text { demand }\end{array}$ & $\begin{array}{c}\text { World growth } \\
\text { surges }\end{array}$ \\
\hline Probabilità scenario & $15 \%$ & $10 \%$ & $2 \%$ & $10 \%$ & $53 \%$ \\
\hline Severity & & & & & $10 \%$ \\
\hline
\end{tabular}

Tabella 3 - Probabilità di realizzazione degli Scenari Oxford Economics.

I risultati della simulazione sono poi riportati nel Grafico 1.

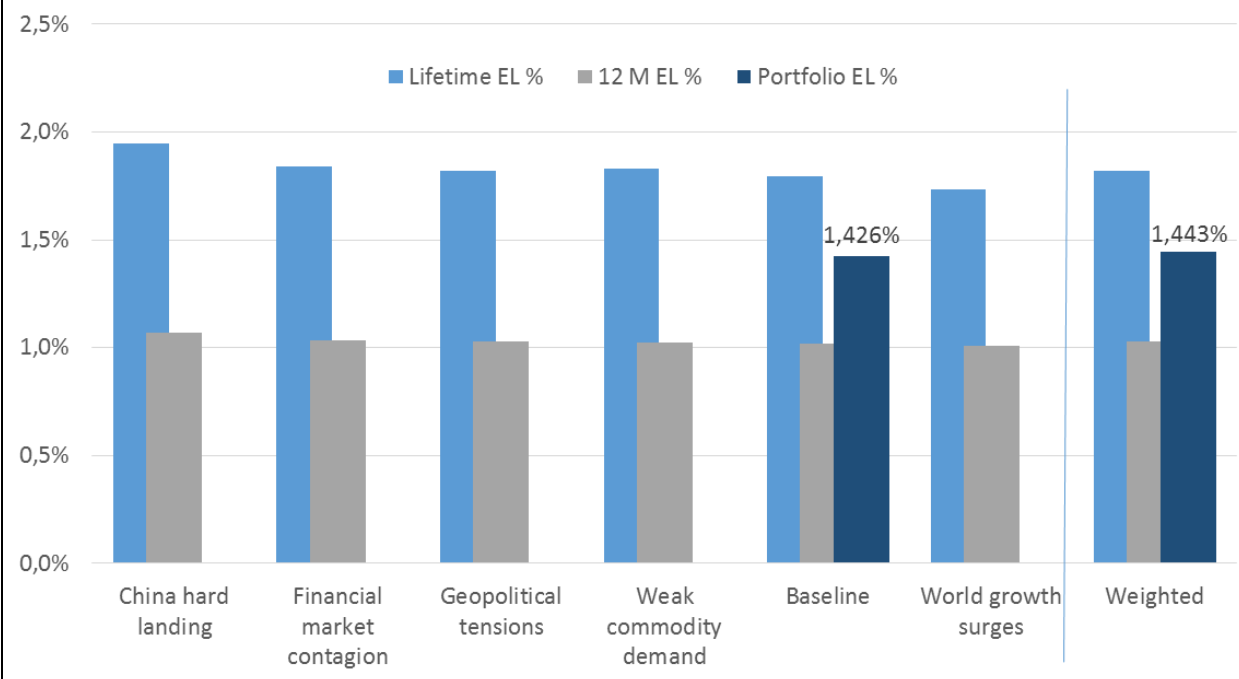

Grafico 1 - Confronto dei valori di ECL per la Simulazione su scenari Oxford Economics.

La considerazione di scenari multipli nel calcolo delle ECL evidenzia un add-on di circa $+2 b p s$ in termini di Portfolio coverage (Portfolio EL \%) rispetto allo scenario Baseline più probabile.

La esiguità dell'effetto di non linearità stimato con questa simulazione è significativa, sia perché rilevata su un set di scenari articolato e coerente sia in ragione della elevata probabilità associata complessivamente a scenari più severi dello scenario Baseline, pari a $37 \%$.

Al fine di approfondire i potenziali impatti della non linearità a fronte di un diverso set di scenari ed effettuare una analisi di sensitivity della non linearità rispetto alla severity degli scenari è stata condotta una seconda simulazione, considerando come input gli scenari forniti dall'EBA e l'upturn scenario di Oxford Economics. Le probabilità di realizzazione sono state assegnate con approccio judgemental, assegnando una probabilità limitata pari al 5\% allo scenario EBA Adverse e considerando una percentuale di realizzazione dello scenario favorevole inferiore a quella assegnata a questo scenario avverso, in coerenza con due considerazioni di fondo:

- $\quad$ coerenza con la maggiore severity dello stress scenario dell'EBA rispetto allo scenario di maggior downturn di Oxford Economics, rispetto a cui pertanto alla probabilità di accadimento è stato assegnato un valore inferiore;

- $\quad$ espressione di una view di incertezza sulle prospettive di recovery dell'economia.

Le probabilità di realizzazione sono riportate in tabella 4.

\begin{tabular}{|l|c|c|c|}
\hline \multicolumn{4}{|c|}{ Simulazione 2 } \\
\hline Scenario & Adverse & Baseline & Favourable \\
\hline Probabilità scenario & $5 \%$ & $93 \%$ & $2 \%$ \\
\hline Severity & & & \\
\hline
\end{tabular}

Tabella 4 - Probabilità di realizzazione degli Scenari combinati EBA/Oxford Economics Upturn. 
I risultati della simulazione sono riportati nel Grafico 2.

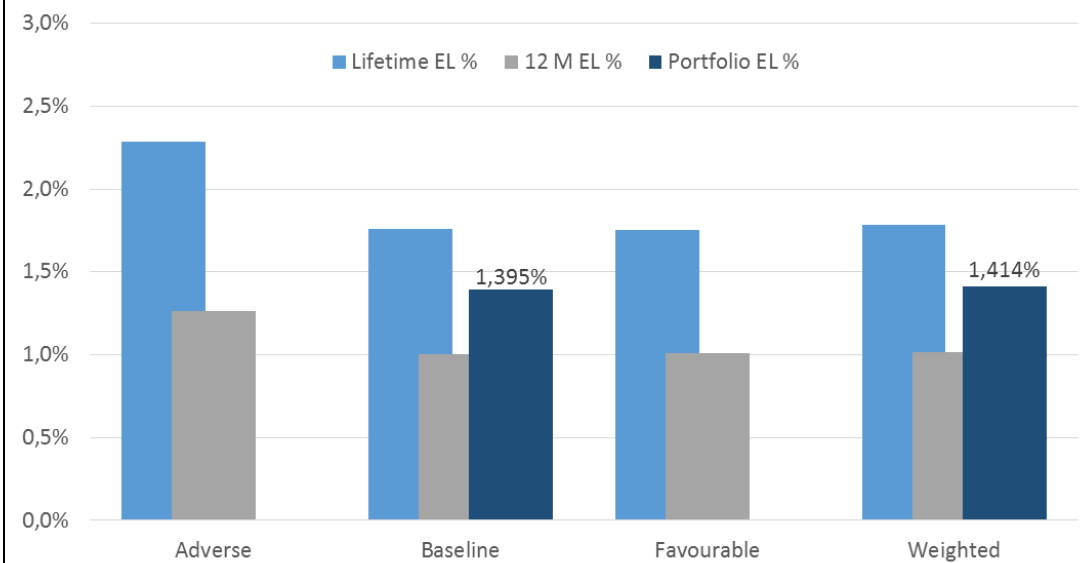

Grafico 2 - Confronto dei valori di ECL per Scenari combinati EBA/Oxford Economics Upturn.

La seconda simulazione conferma l'impatto molto limitato della non linearità in termini di coverage, coerente con quello rilevato nella prima simulazione.

Le stime condizionate a ogni singolo scenario del set sono stati utilizzati ai fini di una successiva analisi di sensitivity, in cui:

- la probabilità dello scenario EBA Adverse è stata incrementata fino al 20\%;

- la probabilità dello scenario favorevole (Oxford Economics Upturn) è stata incrementata proporzionalmente, su livelli sempre inferiori a quella dello scenario EBA Adverse;

- la probabilità dello scenario Baseline è stata ridotta in relazione alle probabilità assegnate agli scenari favorevole e avverso.

I risultati a livello di portafoglio sono riportati nel Grafico 3.

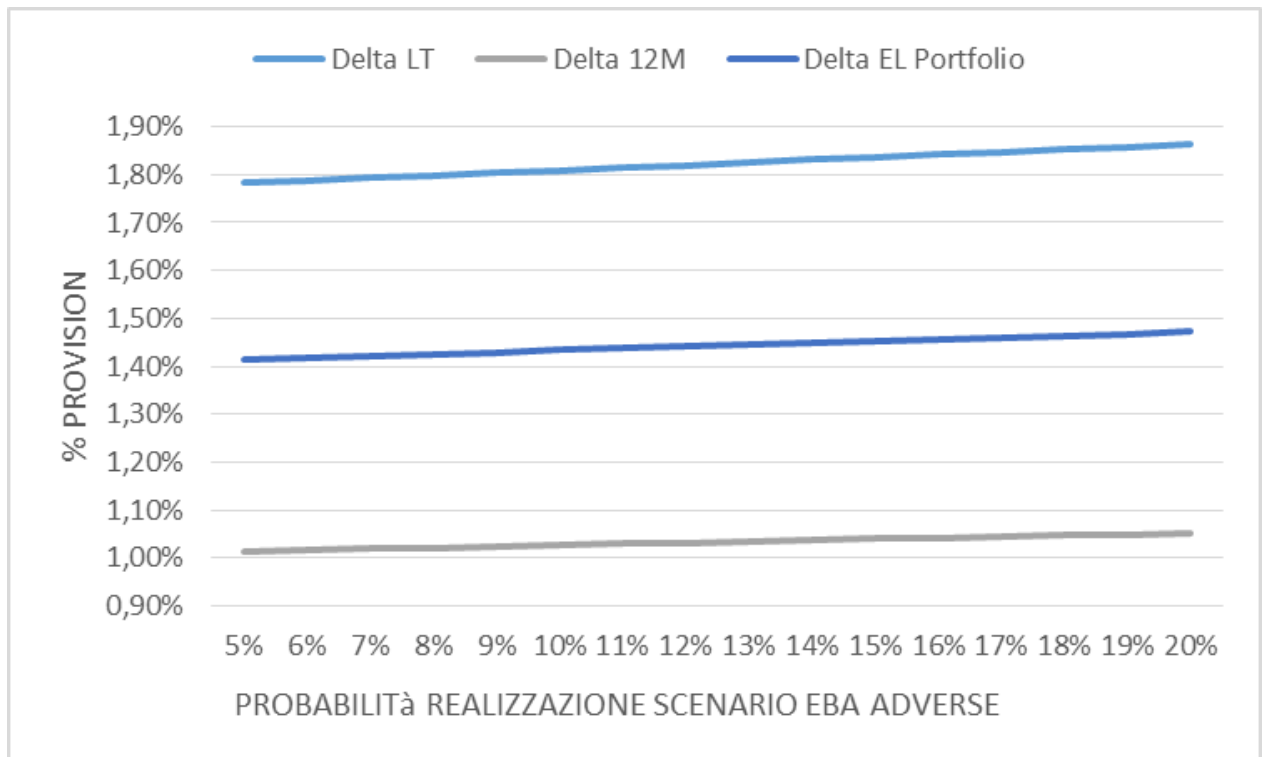

Grafico 3 - Andamento del Coverage Lifetime/12M/Portfolio rispetto alla probabilità di realizzazione dello scenario Adverse (Sensitivity).

Si osserva che un incremento della probabilità dello scenario adverse dell' $1 \%$ implica mediamente un incremento di coverage Lifetime di circa $0,5 \mathrm{bps}$, un incremento di coverage $12 \mathrm{M}$ di $0,3 \mathrm{bps}$ ed un incremento di coverage di portfolio pari a 0,38 bps. Complessivamente l'effetto evidenziato della non linearità appare più significativo rispetto alla 
simulazione basata sul set di scenari di Oxford Economics, fatto che si può ascrivere alla maggiore severity dello stress scenario dell'EBA rispetto allo scenario di maggior downturn di Oxford Economics.

L'analisi è stata poi ulteriormente approfondita a livello di segmento e ha evidenziato i risultati riportati nel Grafico 4, nel Grafico 5 e nel Grafico 6.

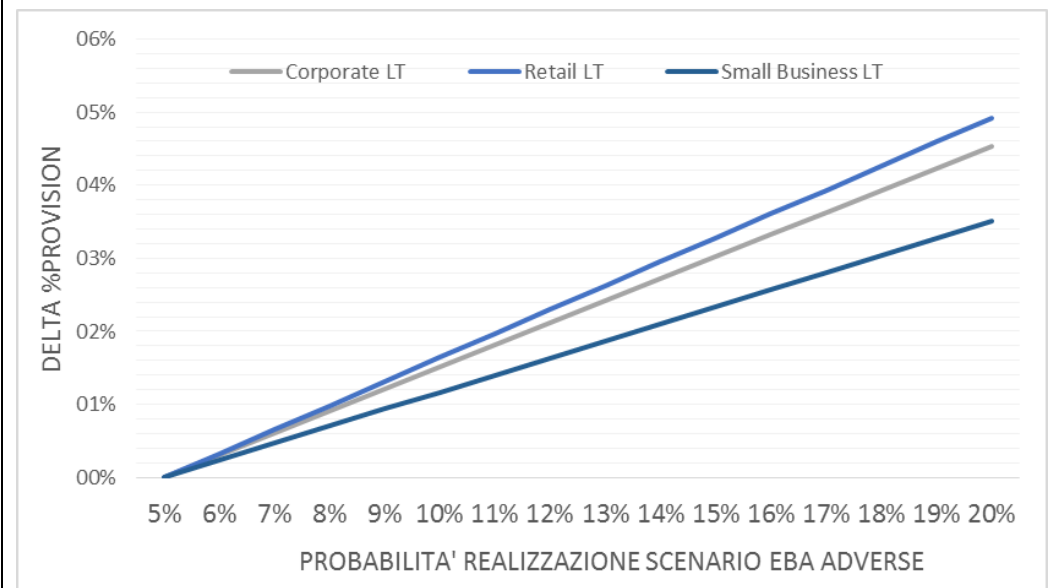

Grafico 4 - Variazione del Coverage Lifetime differenziata per segmento, rispetto alla probabilità di realizzazione dello scenario Adverse (Sensitivity).

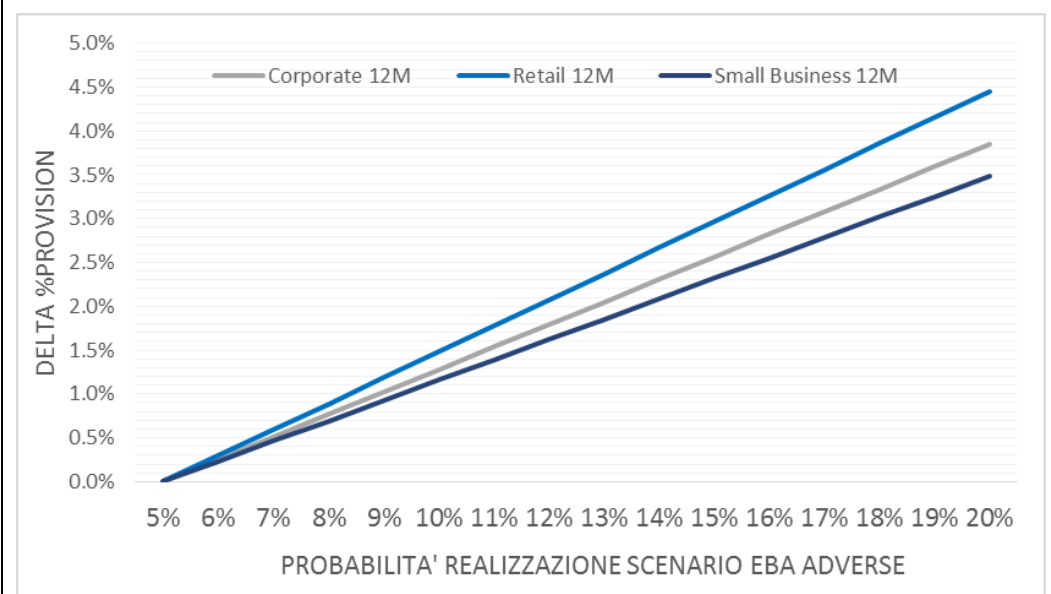

Grafico 5 - Variazione del Coverage 12M differenziata per segmento, rispetto alla probabilità di realizzazione dello scenario Adverse (Sensitivity).

I risultati riportati evidenziano come un incremento delle percentuali dello scenario adverse ha un effetto incrementale sulle provisions differenziato a seconda del segmento di portafoglio. In particolare, l'add-on effect da non linearità relativo ad un incremento dell' $1 \%$ per la probabilità di realizzazione dello scenario EBA adverse, risulta pari a $0,26 \%$ per le ECL a 12 mesi e pari a 0,30\% per le ECL Lifetime per il segmento Corporate, 0,23\% per le ECL a 12 mesi e 0,23\% per le ECL Lifetime per il segmento Small Business, 0,30\% per le ECL a 12 mesi e 0,33\% per le ECL Lifetime per il segmento Retail. 


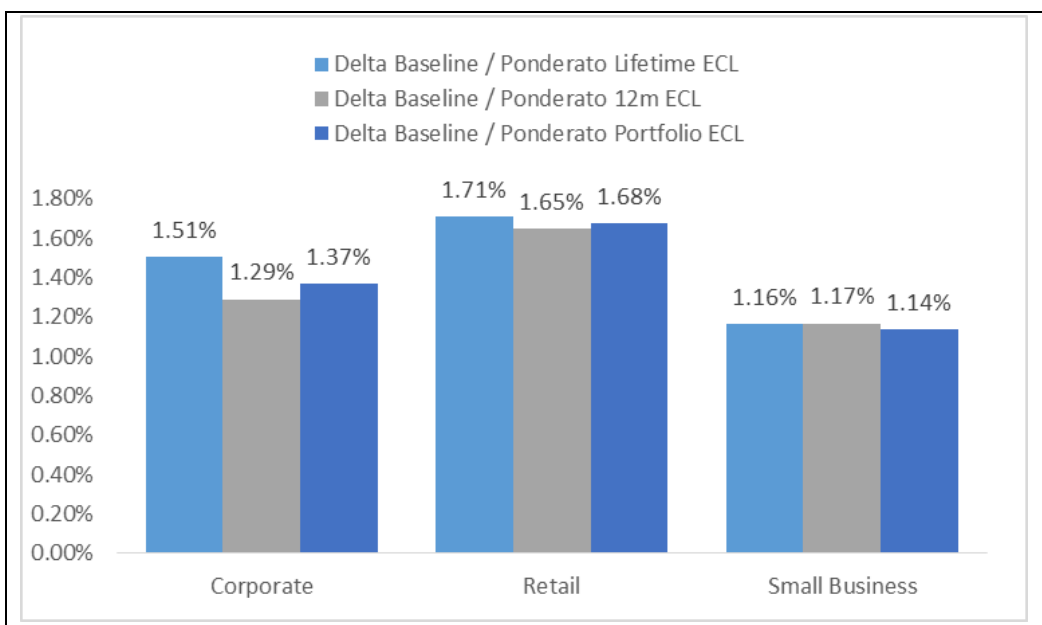

Grafico 6 - Variazione percentuale del Coverage Lifetime/12M/Portfolio, differenziata per segmento fra scenario Baseline e scenario Ponderato Scenari combinati EBA/Oxford Economics Upturn (probabilità di realizzazione come riportato in Tabella 4).

Infine, a conclusione dell'analisi di sensitivity è stato analizzato il differenziale di provisions fra scenario Baseline e risultato della ponderazione degli scenari nel caso limite di valutazione della probabilità di realizzazione dello scenario EBA Adverse al 20\%. I risultati sono riportati nel Grafico 7.

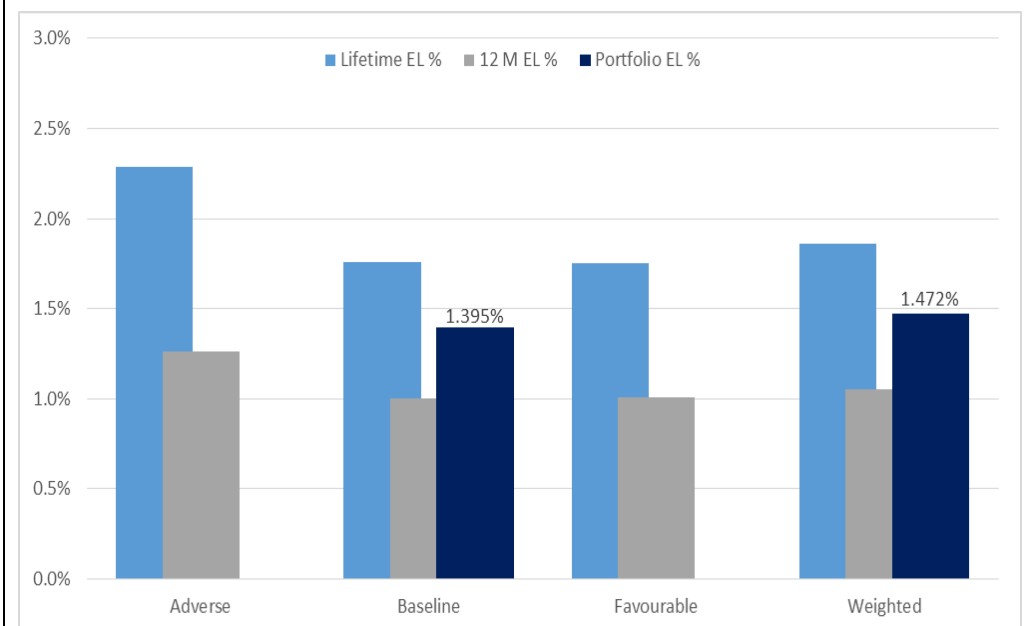

Grafico 7 - Confronto dei valori di ECL per Scenari combinati EBA/Oxford Economics Upturn (probabilità di realizzazione ridefinite assegnando una probabilità del $20 \%$ allo scenario EBA Adverse).

In particolare, in questa situazione estremante le provision complessive di portafoglio aumentano di 8 bps mentre il differenziale percentuale fra Portfolio EL da scenario Baseline e ponderazione degli scenari si attesta al 5,5\%.

Si rileva inoltre quindi in generale che, in termini relativi, l'effetto di non linearità misurato per differenza fra le ECL da scenario Baseline e le ECL calcolate con approccio "Multiple Scenarios" scenario ponderato risulta nella maggior parte dei casi superiore per la stima Lifetime rispetto a quella a 12 mesi.

\section{Conclusioni}

L'analisi, da un lato, del nuovo requisito contabile IFRS9 e dei requisiti tecnici ITG collegati e, dall'altro, dei documenti di guidance in ambito Vigilanza e Audit conferma che a fini di compliance e robusta implementazione è necessario effettuare test periodici di non linearità ed eventualmente effettuare il calcolo degli accantonamenti IFRS9 scontando l'effetto di scenari multipli. L'applicazione presentata in questo documento costituisce un contributo applicativo al dibattito sul tema nel settore bancario con l'utilizzo, la probabilizzazione e la combinazione di scenari esterni proprietari e regolamentari per l'effettuazione dei test di non linearità e del calcolo degli accantonamenti secondo il nuovo standard.

Le analisi e simulazioni presentate evidenziano da un lato l'effetto della non linearità che si evidenzia con l'utilizzo di scenari esterni proprietari, dall'altro le sensitivity degli accantonamenti IFRS9 a severity e probabilità degli scenari 
avversi considerati. È importante sottolineare al riguardo che le necessarie ipotesi e semplificazioni adottate possono essere considerate per gli specifici fini di questo studio meno penalizzanti rispetto a obiettivi di compliance del calcolo puntuale in quanto sono scontate ceteris paribus in ogni scenario di riferimento.

D'altro canto, la rilevanza e articolazione di modelli e ipotesi presentati in questo studio costituisce una testimonianza concreta che la gestione degli scenari multipli a fini di IFRS9 Impairment secondo un framework complessivo strutturato rappresenta a oggi una challenge oggettiva per gli intermediari di tutte le dimensioni, da un punto di vista metodologico, implementativo e di Governance.

\section{Emilio Maffi, Daniele Monzali e Giuseppe Quaglia}

\section{Bibliografia e Riferimenti.}

1. EY, “Applying IFRS - ITG discusses IFRS 9 impairment issues at December 2015 ITG meeting” Dicembre 2015.

2. IASB, "IFRS9 Financial Instruments" n. 5.5.17 (a), Luglio 2014.

3. IASB, "IFRS9 Financial Instruments" n. 5.5.4, Luglio 2014.

4. IASB, "IFRS9 Financial Instruments" n. 5.5.17 (c), Luglio 2014.

5. IASB, "IFRS9 Financial Instruments" n. 5.5.11, Luglio 2014.

6. ITG, “Agenda Paper 1" n. 28, Dicembre 2015.

7. ITG, "Agenda Paper 1" n. 30, Dicembre 2015.

8. ITG, "Agenda Paper 1" n. 54, Dicembre 2015.

9. ITG, "Meeting Summary" n. 59, Dicembre 2015.

10. ITG, "Meeting Summary" n. 55, Dicembre 2015.

11. ITG, "Agenda Paper 1" n. 32, Dicembre 2015.

12. EBA/CP/2016/10, "Draft Guidelines on credit institutions' credit risk management practices and accounting for expected credit losses" n. 6, Ottobre 2016.

13. EBA/CP/2016/10, "Draft Guidelines on credit institutions' credit risk management practices and accounting for expected credit losses" n. 20, Ottobre 2016.

14. EBA/CP/2016/10, "Draft Guidelines on credit institutions' credit risk management practices and accounting for expected credit losses" n. 131, Ottobre 2016.

15. IIF SAG, "ECL accounting implementation issues" Aprile 2016.

16. IIF SAG, "ECL accounting implementation issues" n. (i), Aprile 2016.

17. GPPC, "The implementation of IFRS 9 impairment requirements by banks" n. 2.8.2.1, Giugno 2016.

18. GPPC, "The implementation of IFRS 9 impairment requirements by banks" n. 2.8.2.2, Giugno 2016.

19. GPPC, "The implementation of IFRS 9 impairment requirements by banks" n. 2.8.4.1, Giugno 2016.

20. GPPC, "The implementation of IFRS 9 impairment requirements by banks" n. 2.8.4.2, Giugno 2016.

21. ITG, "Meeting Summary" Dicembre 2015.

22. ITG, "Meeting Summary" n. 50, Dicembre 2015.

23. Oxford Economics, "Global Country Forecast" Scenari aggiornati a Aprile 2016.

24. European Systemic Risk Board, "Adverse macro-financial scenario for the EBA 2016 EU-wide bank" Gennaio 2016.

25. Banca d'Italia, "http://www.bancaditalia.it/statistiche/basi-dati/bds/index.html” 2016.

26. Banca d'Italia, "Supplementi al Bollettino Statistico-Indicatori Monetari e Finanziari” Moneta e Banche, vol. 10, n. 22, Maggio 2016.

Questa pubblicazione contiene informazioni di sintesi ed è pertanto esclusivamente intesa a scopo orientativo; non intende essere sostitutiva di un approfondimento dettagliato o di una valutazione professionale. EYGM Limited o le altre member firm dell'organizzazione globale EY non assumono alcuna responsabilità per le perdite causate a chiunque in conseguenza di azioni od omissioni intraprese sulla base delle informazioni contenute nella presente pubblicazione. Per qualsiasi questione di carattere specifico, è opportuno consultarsi con un professionista competente della materia. 


\section{L'esposizione al rischio di tasso di interesse del portafoglio bancario: quali implicazioni per le strategie di Asset \& Liability Management?}

di Igor Gianfrancesco ${ }^{1}$

\section{ABSTRACT}

Maturity transformation lies at the essence of banking business and represents the main source of interest rate risk in the banking book (IRRBB). Sound management of this risk can improve a bank's profitability and act as a fundamental driver for the shareholders' value creation process. Nevertheless, risk arising from fluctuations of interest rates can pose a significant threat to a bank's ability to generate earnings and to the adequacy of its capital endowment. Therefore, an effective risk management process maintaining interest rate risk within prudential levels is essential for the overall banking stability, due to the systemic nature of this risk.

Within this context, this paper aims to shed more light on the factors explaining the bank's risk exposure to IRRBB in order to allow credit institutions to define the most suitable Asset and Liability Management (ALM) strategies, given the current risk profile and the risk targets set within their Risk Appetite Framework $(R A F)$. This issue has become crucial in the last period, where as a result of the sovereign debt crisis, there has been a change in banks' ALM strategies that has led to an increase in the share of the banking book represented by bonds. Furthermore, the low level of interest rates has affected banks' risk exposure by determining the so-called risk neutrality phenomenon, according to which, by applying both the positive and negative scenarios of changes in interest rates set within the regulatory methodologies, a bank experiences an increase in its equity economic value.

Referred to a sample of 40 Italian commercial banks over the period 2006-2015, the analysis confirms the importance of a correct understanding of all the factors explaining the nature of banks' risk exposure. They can be essentially brought back to the term structure of net positions across the time bands of the maturity ladder, which is the result of banks' ALM strategies, and to the scenario of annual changes in the key-rates, depending on which methodology is adopted and the application of the non-negativity constraint. The definition of the optimal mix of assets and liabilities, with regard to both pricing (fixed vs. floating rate) and maturity (short-vs. long-term) conditions, of the adequate amount of securities to be held in the portfolio and of the most appropriate hedging policies is crucial to have a sound term structure of balance sheet items. This would allow to ensure, from a micro perspective, the stability of each bank and then, from a macro perspective, the stability of the system as a whole.

\section{Introduzione}

Il presente contributo si propone di analizzare l'esposizione al rischio di tasso di interesse del portafoglio bancario di un campione di 40 banche italiane nel periodo 2006-2015 al fine di fornire le linee guida funzionali alla comprensione delle determinanti dell'esposizione al rischio in questione e all'identificazione delle strategie di Asset \& Liability Management $(A L M)$ più appropriate per il raggiungimento degli obiettivi di rischio definiti in sede di Risk Appetite Framework (RAF). La questione è di particolare rilevanza soprattutto nell'ultimo periodo, dove, a partire dalla crisi del debito sovrano, si è re gistrato un mutamento nelle strategie di $A L M$ delle banche che ha condotto ad un maggior peso sul totale attivo di bilancio dei titoli iscritti nel portafoglio bancario. A ciò si aggiunge il basso livello dei tassi di interesse, che, oltre a influenzare le stesse strategie di $A L M$, ha impattato sulle modalità di calcolo dell'indicatore di rischio regolamentare determinando, a seguito dell'applicazione del vincolo di non negatività, degli effetti distorsivi che hanno dato luogo al fenomeno della neutralità al rischio. Si tratta, in altri termini, di banche non esposte al rischio di tasso di interesse del portafoglio bancario a seguito dell'applicazione delle due metodologie di calcolo proposte dal quadro normativo di vigilanza prudenziale.

Il proseguo della trattazione è strutturato come segue: il paragrafo 2 fornisce una rassegna della normativa di vigilanza prudenziale e della letteratura di riferimento; il paragrafo 3 richiama le modalità di calcolo dell'indicatore di rischio regolamentare secondo il dettato normativo attualmente in vigore; il paragrafo 4 si focalizza sulle differenti tipologie di esposizioni al rischio che una banca può assumere nell'ambito della propria operatività (banca esposta a variazioni in aumento dei tassi di interesse, banca esposta a variazioni in diminuzione dei tassi di interesse e banca neutrale al rischio); il paragrafo 5 descrive le differenti modalità di impatto del portafoglio titoli sull'esposizione complessiva al rischio; il paragrafo 6 fornisce le evidenze empiriche nel periodo 2006-2015; il paragrafo 7 analizza le determinanti dell'esposizione al rischio; il paragrafo 8 descrive le possibili strategie di $A L M$ basate sull'utilizzo di strumenti derivati di copertura; e, infine, il paragrafo 9 conclude con le relative implicazioni di policy.

\section{Una rassegna della normativa prudenziale e della letteratura di riferimento}

L'attenzione verso il rischio di tasso di interesse del portafoglio bancario ha assunto una certa rilevanza a seguito della crisi delle Savings and Loans americane degli anni '80. Subito dopo la crisi, infatti, gli economisti della Federal Reserve svilupparono il modello Economic Value Model (EVM), adottando per la misurazione di tale tipologia di rischio la prospettiva del valore economico. Il modello è descritto in Houpt e Embersit (1991) e, successivamente, analizzato in Wright e Houpt

\footnotetext{
${ }^{1}$ Responsabile Ufficio Risk Management presso Extrabanca. Titolare di un contratto di insegnamento integrativo presso la Cattedra di "Economia degli Intermediari Finanziari (corso progredito)" Università LUISS Guido Carli di Roma. Le opinioni espresse sono proprie dell'Autore e non vincolano in alcun modo le Istituzioni di appartenenza.
} 
(1996), Sierra e Yeager (2004) e Sierra (2009). Nel 2004 il Comitato di Basilea (Basel Committee on Banking Supervision $B C B S$ ) ha emanato la versione definitiva del documento tecnico intitolato "Principles for the management of the interest rate risk", che contiene 15 principi per la misurazione e la gestione del rischio in questione e una metodologia di misurazione basata sulla stessa logica sottostante il modello sviluppato dalla FED. In particolare, la metodologia proposta dal Comitato di Basilea: i) prevede la ripartizione dei valori contabili delle attività e delle passività di bilancio sensibili ai tassi di interesse in fasce temporali a cui sono associati specifici coefficienti di duration; ii) prende in considerazione sia una variazione parallela dei tassi di interesse di +/-200 punti base, sia il $1^{\circ}$ e il $99^{\circ}$ percentile delle variazioni dei tassi di interesse su base annua registrate in un periodo di osservazione di 6 anni (nel seguito della trattazione indicati, rispettivamente, con "scenario parallelo" e "metodo dei percentili"); iii) calcola un indicatore di rischio dato dal rapporto tra la variazione di valore economico del portafoglio bancario, a seguito della scenario di variazione dei tassi di interesse ipotizzato, e i fondi propri; e, iv) stabilisce, infine, che le Autorità di vigilanza devono prestare particolare attenzione a quelle banche che presentano un livello dell'indicatore di rischio superiore al $20 \%$.

Nell'Accordo sul Capitale del giugno 2006, il Comitato di Basilea, pur ritenendo il rischio di tasso di interesse del portafoglio bancario potenzialmente significativo e, quindi, tale da richiedere un'adeguata copertura patrimoniale, ha deciso di inserire il rischio in questione nel secondo pilastro non prevedendo, quindi, un apposito requisito minimo regolamentare. Le motivazioni alla base di questa scelta sono riconducibili ai commenti pervenuti dal settore bancario e alle ulteriori analisi svolte dallo stesso Comitato che hanno evidenziato la considerevole eterogeneità tra le banche attive a livello internazionale in relazione sia alla natura del rischio sottostante, sia ai relativi procedimenti di gestione e monitoraggio. Nell'ottobre dello stesso anno, inoltre, il Comitato delle Autorità Europee di Vigilanza Bancaria (Committee of European Banking Supervision $C E B S$ ) ha emanato il documento tecnico intitolato "Technical aspects of the management of interest rate risk arising from non-trading activities under the supervisory review process" che fornisce delle linee guida indirizzate sia alle banche sia alle Autorità di vigilanza nazionali riguardanti, rispettivamente, l'implementazione del processo di determinazione di adeguatezza patrimoniale (Internal Capital Adequacy Assessment Process - ICAAP) e di revisione e valutazione prudenziale (Supervisory Review and Evaluation Process - SREP). Le banche pongono, quindi, a presidio del rischio in questione una dotazione di capitale interno determinata in sede ICAAP. Successivamente, nel 2009 il Comitato di Basilea ha pubblicato il documento tecnico intitolato "Range of practices and issues in economic capital frameworks" in cui viene fornita una rassegna delle best practices adottate dalle banche per il calcolo del capitale economico associato alle varie tipologie di rischi. In relazione al rischio di tasso di interesse del portafoglio bancario l'attenzione si è focalizzata, principalmente, sulla modellizzazione delle opzionalità implicite di alcune poste di bilancio e sui possibili sviluppi della metodologia basata sullo scenario parallelo di +/200 punti base. La Banca d'Italia ha recepito le indicazioni fornite dal Comitato di Basilea nelle Nuove disposizioni di vigilanza prudenziale per le banche emanate nel dicembre 2006, che, per quanto riguarda il rischio in esame, sono state oggetto di modifiche e ulteriori integrazioni con il sesto aggiornamento del 27 dicembre 2010. Infine, nel dicembre 2013 la nuova Circolare 285 emanata dalla stessa Banca d'Italia, in sostituzione della Circolare 263, ha lasciato invariato il quadro regolamentare sul rischio di tasso di interesse del portafoglio bancario. Il modello proposto dal Comitato di Basilea è stato oggetto di analisi da parte di Fiori e Iannotti (2007), Entrop et al. (2008), Entrop et al. (2009), Esposito et al. (2013), Abdymomunov e Gerlach (2014), Cocozza et al. (2015a) e (2015b).

Recentemente nel maggio del 2015 l'Autorità Bancaria Europea (European Banking Authority - EBA) ha aggiornato le linee guida emanate dal CEBS nel 2006 mediante un nuovo documento tecnico intitolato "Guidelines on the management of interest rate risk arising from non-trading activities" e nello stesso anno il Comitato di Basilea ha avviato un processo di consultazione finalizzato a rivedere il framework normativo di riferimento che ha condotto alla pubblicazione nel mese di aprile 2016 del documento tecnico intitolato "Interest rate risk in the banking book". La proposta di riforma del Comitato conferma la collocazione del rischio in questione nell'ambito del secondo pilastro di Basilea data la natura eterogenea dello stesso rafforzandone, mediante un aggiornamento dei relativi principi, il quadro normativo di riferimento. Il nuovo documento tecnico introduce di nuovi scenari di variazione su base annua dei tassi di interesse con cui valutare l'esposizione al rischio, fornisce specifiche indicazioni circa la modellizzazione delle poste di bilancio caratterizzate da elementi di opzionalità e definisce, nel dettaglio, le informazioni da fornire al mercato nell'ambito dell'attività di disclosure. È stata, inoltre, rivista la soglia di attenzione utilizzata ai fini dell'individuazione delle banche anomale che diventa più restrittiva passando dal $20 \%$ al $15 \%$.

\section{L'attuale quadro normativo di vigilanza prudenziale: un quadro di sintesi}

La misurazione dell'esposizione al rischio di tasso di interesse del portafoglio bancario viene effettuata sulla base dell'algoritmo semplificato definito nel Titolo III, Capitolo 1, Allegato C della Circolare della Banca d'Italia n.285 del dicembre 2013. Il modello in questione richiede per ogni singola valuta rilevante ${ }^{2}$ e per l'aggregato delle valute non rilevanti il calcolo per ciascuna fascia temporale della matrice per scadenza e per data di riprezzamento di una posizione netta ottenuta sommando le attività e passività che scadono o si riprezzano nell'orizzonte temporale in questione. Specifici criteri sono definiti per particolari poste di bilancio tra cui i depositi a vista che sono allocati per il $25 \%$ nella fascia temporale a vista e la rimanente parte nelle varie fasce temporali che coprono un orizzonte di 5 anni in proporzione al numero di mesi in esse

\footnotetext{
${ }^{2}$ In base al dettato normativo di vigilanza prudenziale si considerano rilevanti le valute il cui peso misurato come quota sul totale attivo oppure sul passivo del portafoglio bancario è superiore al $5 \%$.
} 
contenute $^{3}$. Le posizioni nette cosi ottenute sono poi moltiplicate per un coefficiente di duration approssimata e per la variazione ipotizzata dei tassi di interesse. In simboli:

$$
\Delta P N_{i}=P N_{i} D M_{i} \Delta r_{i}
$$

dove:

- $\mathrm{PN}_{\mathrm{i}}$ e $\Delta \mathrm{PN}_{\mathrm{i}}$ rappresentano, rispettivamente, la posizione netta e la posizione netta ponderata relative alla i-esima fascia temporale;

- $\mathrm{DM}_{\mathrm{i}}$ è la duration modificata approssimata associata alla i-esima fascia temporale;

- $\Delta \mathrm{r}_{\mathrm{i}}$ è la variazione ipotizzata del tasso di interesse associato alla i-esima fascia temporale.

La (1.) è sostanzialmente analoga alla nota relazione che lega la variazione del prezzo di un titolo a tasso fisso e scadenza determinata alla variazione del suo tasso interno di rendimento a scadenza mediante la duration modificata del titolo stesso. L'unica differenza è che nella (1.) non compare il segno meno nel membro di destra dell'equazione. Di conseguenza, un valore positivo (negativo) di $\Delta \mathrm{PN}_{\mathrm{i}}$ corrisponde a una riduzione (aumento) di pari importo del valore economico della posizione netta $i$-esima. Per ciascuna valuta rilevante e per l'aggregato delle valute non rilevanti $(j=1, \ldots k)$ le posizioni nette ponderate positive ottenute in corrispondenza delle varie fasce temporali $(i=1, \ldots n)$ sono sommate tra loro al fine di ottenere una stima della variazione del valore economico portafoglio bancario $(\Delta \mathrm{VE})$ a seguito dello scenario di variazione dei tassi di interesse ipotizzato. In simboli:

$$
\Delta V E=\sum_{j=1}^{k} \sum_{i=1}^{n} \Delta P N_{i j} \quad s e \sum_{i=1}^{n} \Delta P N_{i j}>0
$$

Il valore così ottenuto è rapportato al valore dei fondi propri (FP) al fine di ottenere un indicatore di rischio (IR) la cui soglia di attenzione è fissata, al $20 \%$. In simboli:

$$
I R=\frac{\Delta V E}{F P}
$$

In base a quanto disposto dalle Disposizioni di vigilanza prudenziale per le banche (Titolo III - Capitolo 1- Sezione II della Circolare n.285/2013 emanata dalla Banca d'Italia) tutte le banche, indipendentemente dalla classe di appartenenza, dalla metodologia utilizzata e dalle variazioni stimate/scenari prescelti per calcolare il capitale interno in condizioni ordinarie/di stress, valutano l'impatto di una variazione ipotetica dei tassi di interesse pari a $+/-200$ punti base sull'esposizione al rischio di tasso di interesse del portafoglio bancario. Nel caso in cui si determini una riduzione del valore economico della banca superiore al 20\% dei fondi propri, la Banca d'Italia approfondisce con la specifica banca i risultati e si riserva di adottare gli opportuni interventi.

Le modifiche apportate nel dicembre 2010 introducono la possibilità di calcolare l'indicatore di rischio per la determinazione del capitale interno in condizioni di operatività ordinaria facendo riferimento alle variazioni annuali dei tassi di interesse rilevati in un periodo di osservazione di 6 anni, considerando, alternativamente, il $1^{\circ}$ (ribasso) o il 99 (rialzo) percentile. Nella stima del capitale interno in ipotesi di stress, invece, le variazioni ipotizzate dei tassi sono determinate sulla base di scenari prescelti dalla banca, oltre a quello della variazione di $+/-200$ punti base. Gli scenari al ribasso, corrispondenti al $1^{\circ}$ percentile e allo scenario parallelo dei -200 punti base, devono, inoltre, essere corretti al fine di rispettare il vincolo di non negatività dei tassi di interesse in base al quale la variazione su base annua dei tassi di interesse ipotizzata non può condurre la struttura vigente dei tassi di interesse alla data di valutazione ad assumere in uno o più nodi valori inferiori a zero.

Ai fini della stima dell'indicatore di rischio si utilizza una struttura per scadenza di 14 tassi di interesse (indicati nel proseguo della trattazione con il termine key-rates) i cui nodi corrispondono alla scadenza a vista e ai punti medi delle altre 13 fasce temporali della matrice per scadenza fornita dalle Autorità di vigilanza. I key-rates utilizzati, in linea con le attuali prassi di settore, sono il tasso EONIA per la scadenza a vista, i tassi Euribor per le scadenze inferiori a 12 mesi e i tassi Swap per le scadenze uguali o superiori a 1 anno. In corrispondenza dei punti medi delle fasce temporali della matrice per scadenza e per data di riprezzamento per i quali non è disponibile un tasso di interesse di mercato il relativo key-rate è ottenuto tramite interpolazione lineare. In linea con quanto disposto in BCBS (2004) e Banca d'Italia (2013) nell'ambito del metodo dei percentili si utilizza ai fini del calcolo delle variazioni su base annua dei key-rates la tecnica delle osservazioni sovrapposte. In base a tale tecnica le variazioni su base annua dei key-rates sono calcolate come differenza tra i livelli osservati in un dato anno e quelli rilevati nell'anno precedente. Utilizzando per ciascun nodo della struttura a termine dei key-rates una serie storica di sei anni di livelli su base giornaliera precedenti la data di valutazione si ottiene una serie storica di cinque anni di variazioni su base annua ${ }^{4}$. Le variazioni su base annua dei key-rates cosi ottenute sono, successivamente corrette per tener conto del vincolo di non negatività dei tassi di interesse ${ }^{5}$.

\footnotetext{
${ }^{3}$ Per un dettaglio sui criteri di allocazione delle varie poste di bilancio nelle fasce temporali della matrice regolamentare si rimanda alla Circolare 272/2008 emanata dalla Banca d'Italia.

${ }^{4} \mathrm{Nel}$ dettaglio per ogni fascia temporale la serie storica delle variazioni su base annua del relativo key-rate è stata calcolata partendo dalla differenza dei livelli registrati, rispettivamente, nell'ultimo giorno dell'anno corrispondente alla data di valutazione e nell'ultimo giorno dell'anno precedente. Si tratta, ovviamente, dei giorni in cui sono disponibili quotazioni di mercato. Successivamente si procede a ritroso calcolando la differenza tra il penultimo giorno disponibile dell'anno di valutazione e quello dell'anno precedente e così via fino a coprire i sei anni di livelli richiesti dalla normativa di vigilanza prudenziale.

${ }^{5}$ Ai fini dell'applicazione del vincolo di non negatività è interessante analizzare l'ipotesi in cui il key-rates vigente alla data di valutazione in corrispondenza di uno specifico nodo è inferiore a zero. In tale ipotesi nel caso di applicazione dello scenario parallelo dei -200 punti base
} 
Nello specifico il metodo dei percentili prevede la determinazione, in corrispondenza di ciascuna fascia temporale, del $1^{\circ}$ e del $99^{\circ}$ percentile della distribuzione storica delle variazioni su base annua del relativo key-rate. In tal modo si ottengono due differenti scenari. Il primo scenario composto per ciascuna fascia temporale da variazioni, in genere, negative dei key-rates; il secondo caratterizzato, invece, per ciascuna fascia temporale, da variazioni positive. Ai fini della stima dell'indicatore di rischio i due scenari sopra definiti sono applicati alle posizioni nette ottenute sulla base dei dati di bilancio relativi alla data di valutazione mediante la (1.). Per ciascun scenario le posizioni nette ponderate così ottenute sono sommate tra loro mediante la (2.) in modo da ottenere una stima della variazione del valore economico del portafoglio bancario, che, rapportata ai fondi propri alla data di valutazione, consente di ottenere, sulla base della (3.), l'indicatore di rischio regolamentare.

L'orizzonte temporale di un anno a cui fare riferimento per la stima dell'esposizione al rischio di tasso di interesse del portafoglio bancario è specificatamente indicato da BCBS (2004) in quanto ritenuto coerente con la capacità degli intermediari di ristrutturare o coprire le esposizioni al fine di mitigare perdite generate da una eccezionale volatilità dei tassi di interesse. Lo stesso documento richiede l'utilizzo di un periodo storico di 5 anni di variazioni su base annua dei tassi di interesse, in quanto ritenuto abbastanza lungo da comprendere i recenti e rilevanti andamenti ciclici dei tassi di interesse. Tale periodo è considerato adeguato anche per alimentare i modelli interni di misurazione basati sul concetto di Value at Risk (VaR) con un intervallo di confidenza del $99 \%$.

\section{Le differenti tipologie di esposizione al rischio}

Data la funzione di trasformazione delle scadenze, basata sul finanziamento di attività a lungo termine con passività a breve e medio termine, una banca dovrebbe risultare esposta a variazioni in aumento dei tassi di interesse. La riduzione del valore economico delle attività a lungo termine, a seguito di un aumento dei tassi di interesse dovrebbe, infatti, risultare maggiore di quella delle passività a breve e medio termine comportando, di conseguenza, una complessiva riduzione del valore economico dei fondi propri. Nella realtà operativa, tuttavia, data l'applicazione dei criteri di allocazione delle varie poste di bilancio, che ne modificano la durata originaria contrattuale nella prospettiva del rischio di tasso di interesse del portafoglio bancario, si rileva la presenza non solo di banche esposte a variazioni in aumento dei tassi di interesse, ma anche di banche esposte a variazioni in diminuzione dei tassi di interesse. L'applicazione delle due metodologie proposte dalla normativa di vigilanza prudenziale, rappresentate, come detto, dallo scenario parallelo dei $+/-200$ punti base e dal metodo dei percentili, su un campione di 40 banche italiane nel periodo 2006-2015 evidenzia, inoltre, che alcune banche sono caratterizzate dal fenomeno della neutralità al rischio. Si tratta, nello specifico, di banche per le quali si rileva un incremento del valore economico dei fondi propri in corrispondenza di entrambi gli scenari di variazione su base annua dei tassi di interesse utilizzati nell'ambito delle metodologie in questione ai fini della stima dell'indicatore di rischio regolamentare. La neutralità al rischio può verificarsi per una data banca a seguito dell'applicazione di una delle due metodologie regolamentari o di entrambe.

Nel proseguo sono illustrate nel dettaglio le differenti tipologie di esposizione al rischio che una banca può assumere: esposta a variazioni in aumento dei tassi di interesse, esposta a variazioni in diminuzione dei tassi di interesse e neutrale al rischio. Ai fini dell'analisi si fa riferimento alla matrice per scadenza e data di riprezzamento pubblicata nel bilancio di esercizio di fine anno nella Parte E della nota integrativa intitolata "Informazioni sui rischi e sulle relative politiche di copertura". Per i dati relativi alla dotazione patrimoniale si è fatto riferimento, invece, alla parte E della nota integrativa intitolata "Informazioni sul patrimonio". La matrice per scadenza e per data di riprezzamento riportata nei bilanci bancari presenta, tuttavia, un numero di fasce temporali inferiori a quelle di cui alla matrice regolamentare. I dati sono stati, quindi, redistribuiti nelle varie fasce temporali della matrice regolamentare in proporzione del numero di mesi contenenti nelle stesse. Ad esempio, i volumi contenuti nella fascia temporale "Da 1 mese a 3 mesi" della matrice di bilancio sono stati redistribuiti per $1 / 3$ nella fascia temporale "Fino ad 1 mese" e per il $2 / 3$ nella fascia temporale "Da oltre 1 mese fino a 3 mesi". Per quanto riguarda i tassi di interesse utilizzati nell'analisi si fa riferimento a quanto detto nel paragrafo precedente. Le banche di seguito considerate sono parte del campione analizzato nel proseguo dell'analisi e sono state scelte in quanto rappresentative delle tre differenti tipologie di esposizione al rischio.

\section{Banca esposta a variazioni in aumento dei tassi di interesse}

Una banca è esposta a variazioni in aumento dei tassi di interesse quando in caso di scenario in aumento (diminuzione) dei tassi di interesse la riduzione (incremento) del valore economico delle attività è maggiore di quella (quello) delle passività determinando, di conseguenza, una riduzione (incremento) del valore economico dei fondi propri. In altre parole, usando la terminologia regolamentare, una banca è esposta a variazioni in aumento dei tassi di interesse quando in caso di scenari in aumento (diminuzione) dei tassi di interesse la somma delle posizioni nette ponderate positive è maggiore (minore) della somma, in valore assoluto, delle posizioni nette ponderate negative. Nel caso di una banca esposta a variazioni in aumento dei tassi di interesse la riduzione di valore economico dei fondi propri si registra, in genere, sulle fasce temporali a lungo termine

l'applicazione del vincolo di non negatività impone di considerare una variazione positiva di uguale ampiezza al livello del key-rates in questione in modo tale che lo scenario di variazione dei tassi di interesse considerato ai fini della stima dell'esposizione al rischio conduce la struttura per scadenza dei tassi di interesse vigente alla data di valutazione ad assumere valori non negativi. Nel caso, invece, di adozione del $1^{\circ}$ percentile le variazioni su base annua ottenute mediante la tecnica delle osservazioni sovrapposte sono sommate algebricamente al livello del key-rates vigente alla data di valutazione. Nell'ipotesi in cui si rileva un valore maggiore di zero si considera la variazione ottenuta mediante l'applicazione della tecnica delle osservazioni sovrapposte; in alternativa si considera, invece, una variazione positiva di pari ampiezza al livello dello stesso key-rates, cosi come per lo scenario parallelo dei -200 punti base. 
(oltre i 5 anni), dove sono presenti posizioni nette positive riconducibili, essenzialmente, alla scadenza delle quote capitale dei mutui a tasso fisso e dei titoli a tasso fisso iscritti nel portafoglio bancario che rientrano nell'orizzonte temporale in questione.

La tabella 1 riporta il calcolo dell'indicatore di rischio per la banca A, che risulta esposta a variazioni in aumento dei tassi di interesse. Dalla seconda alla quarta colonna sono indicati, in corrispondenza delle varie fasce temporali della matrice per scadenza, il livello dei key-rates alla data di valutazione, il valore delle posizioni nette e il relativo coefficiente di duration. Nelle colonne successive, per ogni metodologia di misurazione sono riportati lo specifico scenario di variazione su base annua dei key-rates utilizzato ai fini della stima dell'indicatore di rischio con le relative posizioni nette ponderate. Gli scenari di variazione in diminuzione dei key-rates sono corretti per tener conto del vincolo di non negatività. La penultima e l'ultima riga della tabella 1 riportano, rispettivamente, la somma delle posizioni nette ponderate e il corrispondente livello dell'indicatore di rischio. Nel caso in cui la somma delle posizioni nette ponderate è minore di zero si registra un incremento di valore economico dei fondi propri a seguito dello specifico scenario di variazione dei tassi di interesse utilizzato e il livello dell'indicatore di rischio ha, per costruzione, segno negativo.

Tabella 1. Calcolo indicatore di rischio banca A esposta a variazioni in aumento dei tassi di interesse (PN, $\triangle \mathrm{PN}$ e $\Delta \mathrm{VE}$ in migliaia di euro - key-rates e indicatore di rischio in valori percentuali)

\begin{tabular}{|c|c|c|c|c|c|c|c|c|c|c|c|}
\hline \multirow{2}{*}{$\begin{array}{c}\text { Fasce } \\
\text { temporali }\end{array}$} & \multirow{2}{*}{$\begin{array}{l}\text { Livello } \\
\text { key- } \\
\text { rates }\end{array}$} & \multirow{2}{*}{$\begin{array}{l}\text { Posizioni } \\
\text { Nette } \\
\text { (PN) (a) }\end{array}$} & \multirow{2}{*}{$\begin{array}{l}\text { Duration } \\
\text { (b) }\end{array}$} & \multicolumn{4}{|c|}{ Scenario parallelo } & \multicolumn{4}{|c|}{ Metodo dei percentili } \\
\hline & & & & $\begin{array}{c}+2,00 \\
\text { (c) }\end{array}$ & $\begin{array}{c}\Delta \mathbf{P N} \\
(\mathbf{a}) *(\mathbf{b}) *(\mathbf{c})\end{array}$ & $\begin{array}{c}-2,00 \\
(d)\end{array}$ & $\begin{array}{c}\Delta \mathbf{P N} \\
(\mathbf{a}) *(\mathbf{b}) *(\mathbf{d}) \\
\end{array}$ & $\begin{array}{l}99^{\circ} \\
(\mathbf{e})\end{array}$ & $\begin{array}{c}\Delta \mathbf{P N} \\
(\mathbf{a})^{*}(\mathbf{b}) *(\mathbf{e})\end{array}$ & $\begin{array}{l}1^{\circ} \\
\text { (f) }\end{array}$ & $\begin{array}{c}\Delta \mathbf{P N} \\
(\mathbf{a}) *(\mathbf{b}) *(\mathbf{f})\end{array}$ \\
\hline A vista & 0,63 & -874.662 & 0,00 & 2,00 & 0 & $-0,63$ & 0 & 1,31 & 0 & $-0,63$ & 0 \\
\hline Fino a 1m & 0,76 & 478.580 & 0,04 & 2,00 & 380 & $-0,76$ & -145 & 1,24 & 236 & $-0,76$ & -145 \\
\hline $1 \mathrm{~m}-3 \mathrm{~m}$ & 1,18 & 957.160 & 0,16 & 2,00 & 3.039 & $-1,18$ & -1.790 & 1,38 & 2.090 & $-1,18$ & -1.790 \\
\hline $3 m-6 m$ & 1,48 & -120.065 & 0,36 & 2,00 & -864 & $-1,48$ & 638 & 1,33 & -574 & $-1,48$ & 638 \\
\hline $6 \mathrm{~m}-1 \mathrm{a}$ & 1,79 & -135.335 & 0,71 & 2,00 & -1.922 & $-1,79$ & 1.721 & 1,24 & -1.194 & $-1,79$ & 1.721 \\
\hline $1 a-2 a$ & 1,37 & -207.516 & 1,38 & 2,00 & -5.727 & $-1,37$ & 3.920 & 1,19 & -3.414 & $-1,37$ & 3.920 \\
\hline $2 a-3 a$ & 1,35 & -207.516 & 2,25 & 2,00 & -9.338 & $-1,35$ & 6.294 & 1,10 & -5.131 & $-1,35$ & 6.294 \\
\hline $3 a-4 a$ & 1,45 & -207.516 & 3,07 & 2,00 & -12.741 & $-1,45$ & 9.253 & 1,03 & -6.549 & $-1,45$ & 9.253 \\
\hline $4 a-5 a$ & 1,63 & -207.516 & 3,85 & 2,00 & -15.979 & $-1,63$ & 12.995 & 0,98 & -7.815 & $-1,63$ & 12.995 \\
\hline $5 a-7 a$ & 1,91 & 154.732 & 5,08 & 2,00 & 15.721 & $-1,91$ & -15.029 & 0,91 & 7.129 & $-1,91$ & -15.029 \\
\hline $7 a-10 a$ & 2,24 & 232.097 & 6,63 & 2,00 & 30.776 & $-2,00$ & -30.776 & 0,82 & 12.589 & $-1,50$ & -23.081 \\
\hline 10a-15a & 2,57 & 98.111 & 8,92 & 2,00 & 17.503 & $-2,00$ & -17.503 & 0,76 & 6.654 & $-1,19$ & -10.383 \\
\hline $15 a-20 a$ & 2,68 & 98.111 & 11,21 & 2,00 & 21.997 & $-2,00$ & -21.997 & 0,75 & 8.221 & $-1,06$ & -11.703 \\
\hline Oltre i 20a & 2,65 & 98.111 & 13,01 & 2,00 & 25.529 & $-2,00$ & -25.529 & 0,75 & 9.627 & $-1,15$ & -14.640 \\
\hline$\Delta \mathbf{V E}$ & & & & & 68.371 & & -77.947 & & 21.867 & & -41.950 \\
\hline $\mathbf{I R}=\Delta \mathbf{V E} / \mathbf{F P}$ & & & & & 15,54 & & $-17,72$ & & 4,97 & & $-9,54$ \\
\hline
\end{tabular}

Fonte: Elaborazioni dell'Autore su dati di bilancio al 31/12/2011 e dati Datastream.

Il confronto tra i due algoritmi semplificati evidenzia che il valore dell'indicatore di rischio ottenuto mediante l'adozione dello scenario parallelo di +200 punti base $(15,54 \%)$ è maggiore di quello ottenuto mediante l'utilizzo del metodo dei percentili $(4,97 \%)$. Data la struttura di bilancio della banca oggetto di analisi che conduce, in base ai criteri di classificazione, a posizioni nette negative nelle fasce a medio termine (da 1 a 5 anni) e positive in quelle a lungo termine (oltre i 5 anni), gli scenari di variazioni su base annua dei key-rates utilizzati ai fini della stima dell'indicatore di rischio sono alla base di tali differenze. Lo scenario parallelo di +200 punti base presenta, per tutte le fasce temporali della matrice per scadenza, variazioni su base annua dei key-rates di maggiore ampiezza rispetto allo scenario corrispondente dal $99^{\circ}$ percentile. Di conseguenza, lo scenario parallelo di +200 punti base conduce, da un lato, ad un maggior incremento del valore economico dei fondi propri nelle fasce temporali a medio termine e, dall'altro lato, ad una maggiore riduzione del valore economico dei fondi propri nelle fasce temporali a lungo termine rispetto al metodo dei percentili. Il più ampio divario registrato sulle fasce a lungo termine tra le due metodologie regolamentari in termini di variazioni di valore economico dei fondi propri conduce ad un livello dell'indicatore di rischio più elevato nel caso di applicazione dello scenario parallelo dei +200 punti base.

\section{Banca esposta a variazioni in diminuzione dei tassi di interesse}

Una banca è esposta a variazioni in diminuzione dei tassi di interesse quando in caso di scenario in diminuzione (aumento) dei tassi di interesse l'incremento (riduzione) del valore economico delle passività è maggiore di quello delle attività determinando, di conseguenza, una riduzione (incremento) del valore economico dei fondi propri. In altre parole usando la terminologia regolamentare, una banca è esposta a variazioni in diminuzione dei tassi di interesse quando in caso di scenari in diminuzione (aumento) dei tassi di interesse, la somma delle posizioni nette ponderate positive è maggiore (minore) di quella, in valore assoluto, delle posizioni nette ponderate negative. Nel caso di banche esposte a variazioni in diminuzione 
dei tassi di interesse, a differenza di quanto accade per le banche esposte a variazioni in aumento, la riduzione di valore economico dei fondi propri si registra, in genere, nelle fasce temporali a medio termine (da 1 a 5 anni), dove sono presenti posizioni nette negative riconducibili, principalmente, al peso dei depositi a vista, che, come noto, sono allocati in un orizzonte temporale di 5 anni, e alle varie forme tecniche di raccolta a tasso fisso che scadono in tali fasce temporali.

La seguente tabella 2. riporta il caso della banca B che risulta esposta a variazioni in diminuzione dei tassi di interesse. Le strutture per scadenza delle posizioni nette della banca A e della banca B sono alla base della differente tipologia di esposizione al rischio poiché gli scenari di variazione su base annua dei tassi di interesse applicati sono uguali in quanto dipendono esclusivamente dalla metodologia di misurazione adottata. La banca B è caratterizzata, infatti, rispetto alla banca A da una maggior rilevanza delle posizioni nette negative registrate sulle fasce temporali a medio termine rispetto a quelle positive rilevate sulle fasce a lungo termine.

Tabella 2. Calcolo indicatore di rischio banca $B$ esposta a variazioni in diminuzione dei tassi di interesse (PN, $\triangle \mathrm{PN}$ e $\triangle \mathrm{VE}$ in migliaia di euro - key-rates e indicatore di rischio in valori percentuali)

\begin{tabular}{|c|c|c|c|c|c|c|c|c|c|c|c|}
\hline \multirow{2}{*}{$\begin{array}{c}\text { Fasce } \\
\text { temporali }\end{array}$} & \multirow{2}{*}{$\begin{array}{c}\text { Livello } \\
\text { key- } \\
\text { rates }\end{array}$} & \multirow{2}{*}{$\begin{array}{l}\text { Posizioni } \\
\text { Nette } \\
\text { (PN) (a) }\end{array}$} & \multirow{2}{*}{$\begin{array}{l}\text { Duration } \\
\text { (b) }\end{array}$} & \multicolumn{4}{|c|}{ Scenario parallelo } & \multicolumn{4}{|c|}{ Metodo dei percentili } \\
\hline & & & & $\begin{array}{c}+2,00 \\
(\mathrm{c})\end{array}$ & $\begin{array}{c}\Delta \mathbf{P N} \\
(\mathbf{a}) *(\mathbf{b}) *(\mathbf{c}) \\
\end{array}$ & $\begin{array}{c}-2,00 \\
(d) \\
\end{array}$ & $\begin{array}{c}\Delta \mathbf{P N} \\
(\mathbf{a}) *(\mathbf{b}) *(\mathbf{d})\end{array}$ & $\begin{array}{l}99^{\circ} \\
(\mathbf{e}) \\
\end{array}$ & $\begin{array}{c}\Delta \mathbf{P N} \\
(\mathbf{a}) *(\mathbf{b}) *(\mathbf{e}) \\
\end{array}$ & $\begin{array}{l}1^{\circ} \\
(\mathbf{f})\end{array}$ & $\begin{array}{c}\Delta \mathbf{P N} \\
(\mathbf{a}) *(\mathbf{b}) *(\mathbf{f}) \\
\end{array}$ \\
\hline A vista & 0,63 & -9.827 & 0,00 & 2,00 & 0 & $-0,63$ & 0 & 1,31 & 0 & $-0,63$ & 0 \\
\hline fino a $1 \mathrm{~m}$ & 0,76 & 281.676 & 0,04 & 2,00 & 224 & $-0,76$ & -85 & 1,24 & 139 & $-0,76$ & -85 \\
\hline $\mathbf{1 m - 3 m}$ & 1,18 & 563.352 & 0,16 & 2,00 & 1.788 & $-1,18$ & -1.053 & 1,38 & 1.230 & $-1,18$ & -1.053 \\
\hline $3 m-6 m$ & 1,48 & -105.755 & 0,36 & 2,00 & -761 & $-1,48$ & 562 & 1,33 & -506 & $-1,48$ & 562 \\
\hline $6 \mathrm{~m}-1 \mathrm{a}$ & 1,79 & -104.929 & 0,71 & 2,00 & -1.490 & $-1,79$ & 1.334 & 1,24 & -926 & $-1,79$ & 1.334 \\
\hline $1 a-2 a$ & 1,37 & -160.501 & 1,38 & 2,00 & -4.430 & $-1,37$ & 3.032 & 1,19 & -2.640 & $-1,37$ & 3.032 \\
\hline $\mathbf{2 a}-\mathbf{3 a}$ & 1,35 & -160.501 & 2,25 & 2,00 & -7.223 & $-1,35$ & 4.868 & 1,10 & -3.969 & $-1,35$ & 4.868 \\
\hline $3 a-4 a$ & 1,45 & -160.501 & 3,07 & 2,00 & -9.855 & $-1,45$ & 7.157 & 1,03 & -5.065 & $-1,45$ & 7.157 \\
\hline $4 a-5 a$ & 1,63 & -160.501 & 3,85 & 2,00 & -12.359 & $-1,63$ & 10.051 & 0,98 & -6.045 & $-1,63$ & 10.051 \\
\hline $5 a-7 a$ & 1,91 & 20.893 & 5,08 & 2,00 & 2.123 & $-1,91$ & -2.029 & 0,91 & 963 & $-1,91$ & -2.029 \\
\hline $7 a-10 a$ & 2,24 & 31.340 & 6,63 & 2,00 & 4.156 & $-2,00$ & -4.156 & 0,82 & 1.700 & $-1,50$ & -3.117 \\
\hline $10 a-15 a$ & 2,57 & 8.661 & 8,92 & 2,00 & 1.545 & $-2,00$ & -1.545 & 0,76 & 587 & $-1,19$ & -917 \\
\hline $15 a-20 a$ & 2,68 & 8.661 & 11,21 & 2,00 & 1.942 & $-2,00$ & -1.942 & 0,75 & 726 & $-1,06$ & -1.033 \\
\hline Oltre i 20a & 2,65 & 8.661 & 13,01 & 2,00 & 2.254 & $-2,00$ & -2.254 & 0,75 & 850 & $-1,15$ & -1.292 \\
\hline$\Delta \mathbf{V E}$ & & & & & -22.086 & & 13.939 & & -12.956 & & 17.477 \\
\hline $\mathbf{I R}=\Delta V \mathbf{V E} / \mathbf{F P}$ & & & & & $-11,92$ & & 7,52 & & $-6,99$ & & 9,43 \\
\hline
\end{tabular}

Fonte: Elaborazioni dell'Autore su dati di bilancio al 31/12/2011 e dati Datastream.

Il confronto tra $\mathrm{i}$ due algoritmi semplificati evidenzia che il valore dell'indicatore di rischio ottenuto mediante 1'adozione dello spostamento parallelo di -200 punti base (7,52\%) è minore di quello ottenuto mediante l'utilizzo del metodo dei percentili $(9,43 \%)$. Gli scenari di variazioni su base annua dei key-rates utilizzati ai fini della stima dell'indicatore di rischio sono alla base di tale differenza. È interessante osservare che, data l'applicazione del vincolo di non negatività, le variazioni negative dei key-rates osservate sulle fasce temporali che vanno da quella "A vista" fino a quella "Da oltre 5 anni fino a 7 anni" sono di uguale ampiezza in corrispondenza di entrambi i due algoritmi semplificati. Non si registrano, quindi, su tali fasce temporali, differenze tra le due metodologie regolamentari in termini di variazione di valore economico dei fondi propri. Sulle fasce a lungo termine, invece, lo scenario parallelo di -200 punti base presenta variazioni di maggiore ampiezza, in valore assoluto, rispetto allo scenario associato al $1^{\circ}$ percentile. Lo scenario di -200 punti base fa registrare, quindi, su tali fasce temporali un maggiore incremento di valore economico dei fondi propri che attenua la riduzione registrata, invece, sulle fasce temporale a medio termine. Ciò fa si che l'applicazione dello scenario parallelo conduce ad un livello dell'indicatore di rischio più basso rispetto a quello ottenuto mediante l'adozione del metodo dei percentili.

\section{Il fenomeno della neutralità al rischio}

Una banca è neutrale al rischio se in caso di scenario in aumento (diminuzione) dei tassi di interesse la riduzione (incremento) in valore assoluto del valore economico delle attività è minore (maggiore) di quella delle passività determinando, di conseguenza, un incremento del valore economico dei fondi propri. In altre parole, usando la terminologia regolamentare, una banca è neutrale al rischio quando la somma delle posizioni nette ponderate positive è sempre minore della somma, in valore assoluto, delle posizioni nette ponderate negative, a prescindere dallo scenario di variazione su base annua dei key-rates considerato nell'ambito di ciascuna delle due metodologie regolamentari utilizzate ai fini della stima dell'indicatore di rischio.

La Tabella 3 riporta il caso della banca $\mathrm{C}$ che è neutrale al rischio nell'ipotesi di adozione dello scenario parallelo di +/200 punti base e esposta a variazioni in diminuzione dei tassi di interesse nell'ipotesi di utilizzo del metodo dei percentili. 
L'applicazione del vincolo di non negatività è alla base del fenomeno della neutralità al rischio. Nel dettaglio, nell'ipotesi di utilizzo dello scenario parallelo di -200 punti base non corretto per tener conto del vincolo di non negatività la banca sarebbe stata esposta a variazioni in diminuzione dei tassi di interesse, facendo registrare una riduzione del valore economico dei fondi propri di ammontare, in valore assoluto, esattamente pari all'incremento registrato a seguito dell'applicazione dello scenario parallelo di +200 punti base. L'imposizione del vincolo di non negatività nel caso di adozione dello scenario parallelo dei -200 punti base smorza, di fatto, la riduzione di valore economico associata alle posizioni nette negative registrate nelle fasce a medio termine rendendo la banca neutrale al rischio.

Tabella 3. Indicatore di rischio banca $\mathrm{C}$ neutrale al rischio nel caso di adozione dello scenario parallelo dei $+/-200$ punti base

(PN, $\triangle \mathrm{PN}$ e $\triangle \mathrm{VE}$ in migliaia di euro - key-rates e indicatore di rischio in valori percentuali)

\begin{tabular}{|c|c|c|c|c|c|c|c|c|c|c|c|}
\hline \multirow{2}{*}{$\begin{array}{c}\text { Fasce } \\
\text { temporali }\end{array}$} & \multirow{2}{*}{$\begin{array}{c}\text { Livello } \\
\text { key- } \\
\text { rates }\end{array}$} & \multirow{2}{*}{$\begin{array}{l}\text { Posizioni } \\
\text { Nette } \\
\text { (PN) (a) }\end{array}$} & \multirow{2}{*}{$\begin{array}{l}\text { Duration } \\
\text { (b) }\end{array}$} & \multicolumn{4}{|c|}{ Scenario parallelo } & \multicolumn{4}{|c|}{ Metodo dei percentili } \\
\hline & & & & $\begin{array}{c}+2,00 \\
(\mathrm{c}) \\
\end{array}$ & $\begin{array}{c}\Delta \mathbf{P N} \\
(\mathbf{a}) *(\mathbf{b}) *(\mathbf{c}) \\
\end{array}$ & $\begin{array}{c}-2,00 \\
(d) \\
\end{array}$ & $\begin{array}{c}\Delta \mathbf{P N} \\
(\mathbf{a}) *(\mathbf{b}) *(\mathbf{d})\end{array}$ & $\begin{array}{l}99^{\circ} \\
(\mathbf{e})\end{array}$ & $\begin{array}{c}\Delta \mathbf{P N} \\
(\mathbf{a}) *(\mathbf{b}) *(\mathbf{e}) \\
\end{array}$ & $\begin{array}{l}1^{\circ} \\
\text { (f) }\end{array}$ & $\begin{array}{c}\Delta \mathbf{P N} \\
(\mathbf{a}) *(\mathbf{b}) *(\mathbf{f}) \\
\end{array}$ \\
\hline A vista & 0,63 & 56.046 & 0,00 & 2,00 & 0 & $-0,63$ & 0 & 1,31 & 0 & $-0,63$ & 0 \\
\hline Fino a 1m & 0,76 & 151.484 & 0,04 & 2,00 & 120 & $-0,76$ & -46 & 1,24 & 75 & $-0,76$ & -46 \\
\hline $\mathbf{1 m - 3 m}$ & 1,18 & 302.969 & 0,16 & 2,00 & 962 & $-1,18$ & -567 & 1,38 & 662 & $-1,18$ & -567 \\
\hline $3 m-6 m$ & 1,48 & 30.592 & 0,36 & 2,00 & 220 & $-1,48$ & -163 & 1,33 & 146 & $-1,48$ & -163 \\
\hline $6 m-1 a$ & 1,79 & -9.240 & 0,71 & 2,00 & -131 & $-1,79$ & 117 & 1,24 & -82 & $-1,79$ & 117 \\
\hline $1 a-2 a$ & 1,37 & -109.735 & 1,38 & 2,00 & -3.029 & $-1,37$ & 2.073 & 1,19 & -1.805 & $-1,37$ & 2.073 \\
\hline $\mathbf{2 a - 3 a}$ & 1,35 & -109.735 & 2,25 & 2,00 & -4.938 & $-1,35$ & 3.328 & 1,10 & -2.713 & $-1,35$ & 3.328 \\
\hline $3 a-4 a$ & 1,45 & -109.735 & 3,07 & 2,00 & -6.738 & $-1,45$ & 4.893 & 1,03 & -3.463 & $-1,45$ & 4.893 \\
\hline $4 a-5 a$ & 1,63 & -109.735 & 3,85 & 2,00 & -8.450 & $-1,63$ & 6.872 & 0,98 & -4.133 & $-1,63$ & 6.872 \\
\hline $5 a-7 a$ & 1,91 & 29.958 & 5,08 & 2,00 & 3.044 & $-1,91$ & -2.910 & 0,91 & 1.380 & $-1,91$ & -2.910 \\
\hline $7 \mathbf{a}-10 a$ & 2,24 & 44.938 & 6,63 & 2,00 & 5.959 & $-2,00$ & -5.959 & 0,82 & 2.437 & $-1,50$ & -4.469 \\
\hline $10 a-15 a$ & 2,57 & 13.902 & 8,92 & 2,00 & 2.480 & $-2,00$ & -2.480 & 0,76 & 943 & $-1,19$ & -1.471 \\
\hline $15 a-20 a$ & 2,68 & 13.902 & 11,21 & 2,00 & 3.117 & $-2,00$ & -3.117 & 0,75 & 1.165 & $-1,06$ & -1.658 \\
\hline Oltre i 20a & 2,65 & 13.902 & 13,01 & 2,00 & 3.617 & $-2,00$ & -3.617 & 0,75 & 1.364 & $-1,15$ & -2.074 \\
\hline$\Delta \mathbf{V E}$ & & & & & -3.766 & & -1.575 & & -4.024 & & 3.926 \\
\hline $\mathbf{I R}=\Delta V \mathbf{V E} / \mathbf{F P}$ & & & & & $-1,52$ & & $-0,64$ & & $-1,63$ & & 1,59 \\
\hline
\end{tabular}

Fonte: Elaborazioni dell'Autore su dati di bilancio al 31/12/2011 e dati Datastream.

Il fenomeno della neutralità può verificarsi anche nell'ipotesi di adozione del metodo dei percentili come nel caso, riportato nella Tabella 4, della banca $\mathrm{D}$, che risulta, invece, essere esposta a variazioni in aumento dei tassi di interesse nel caso di utilizzo dello scenario parallelo di +/- 200 punti base. Nel caso specifico della banca D l'imposizione del vincolo di non negatività è alla base della neutralità al rischio. La banca in esame sarebbe, infatti, esposta a variazioni in diminuzione dei tassi di interesse nel caso di applicazione dello scenario relativo al $1^{\circ}$ percentile non corretto per il vincolo di non negatività. L'applicazione di tale scenarioriportato per il 31/12/2011 nella successiva Tabella 12 condurrebbe, infatti, ad un livello dell'indicatore di rischio pari all'8,62\%. L'applicazione del vincolo di non negatività, tuttavia, non è una condizione necessaria affinché si verifichi il fenomeno della neutralità al rischio nel caso di adozione del metodo dei percentili. Tale metodologia, infatti, non conduce ad una esposizione al rischio simmetrica tra il $1^{\circ}$ e il $99^{\circ}$ percentile cosi come avviene, invece, a seguito dell'adozione dello scenario parallelo dei +/-200 punti base. In differenti date di valutazione, la dinamica dei tassi di interesse osservati nei 6 anni precedenti può, infatti, condurre, come effettivamente riscontrato nelle evidenze empiriche relative al periodo 2006-2015 riportate nel paragrafo 6, a particolari scenari associati al $1^{\circ}$ e al $99^{\circ}$ percentile delle distribuzioni delle variazioni su base annua dei key-rates, che, combinati con la struttura per scadenza delle posizioni nette di una banca, generano il fenomeno della neutralità al rischio a prescindere dall'attivazione o meno del vincolo di non negatività. $\mathrm{Ci}$ si riferisce, nello specifico, ai 3 casi di banche neutrali al rischio rilevati al 31/12/2008 dove il livello dei key-rates vigente alla data di valutazione era ad un livello tale da non richiedere l'applicazione del vincolo di non negatività (cfr. successive Tabella da 11 a 13). 
Tabella 4. Indicatore di rischio banca $D$ neutrale al rischio nel caso di adozione del metodo dei percentili

(PN, $\triangle \mathrm{PN}$ e $\triangle \mathrm{VE}$ in migliaia di euro - key-rates e indicatore di rischio in valori percentuali)

\begin{tabular}{|c|c|c|c|c|c|c|c|c|c|c|c|}
\hline \multirow{2}{*}{$\begin{array}{c}\text { Fasce } \\
\text { temporali }\end{array}$} & \multirow{2}{*}{$\begin{array}{c}\text { Livello } \\
\text { key- } \\
\text { rates }\end{array}$} & \multirow{2}{*}{$\begin{array}{l}\text { Posizioni } \\
\text { Nette } \\
\text { (PN) (a) }\end{array}$} & \multirow{2}{*}{$\begin{array}{l}\text { Duration } \\
\text { (b) }\end{array}$} & \multicolumn{4}{|c|}{ Scenario parallelo } & \multicolumn{4}{|c|}{ Metodo dei percentili } \\
\hline & & & & $\begin{array}{c}+2,00 \\
(c) \\
\end{array}$ & $\begin{array}{c}\Delta \mathbf{P N} \\
(\mathbf{a}) *(\mathbf{b}) *(\mathbf{c})\end{array}$ & $\begin{array}{c}-2,00 \\
\text { (d) }\end{array}$ & $\begin{array}{c}\Delta \mathbf{P N} \\
(\mathbf{a}) *(\mathbf{b}) *(\mathbf{d})\end{array}$ & $\begin{array}{l}99^{\circ} \\
(\mathbf{e})\end{array}$ & $\begin{array}{c}\Delta \mathbf{P N} \\
(\mathbf{a}) *(\mathbf{b}) *(\mathbf{e})\end{array}$ & $\begin{array}{l}1^{\circ} \\
(\mathbf{f})\end{array}$ & $\begin{array}{c}\Delta \mathbf{P N} \\
(\mathbf{a}) *(\mathbf{b}) *(\mathbf{f}) \\
\end{array}$ \\
\hline A vista & 0,63 & 739.018 & 0,00 & 2,00 & 0 & $-0,63$ & 0 & 1,31 & 0 & $-0,63$ & 0 \\
\hline Fino a 1m & 0,76 & -47.583 & 0,04 & 2,00 & -38 & $-0,76$ & 14 & 1,24 & -23 & $-0,76$ & 14 \\
\hline 1m-3m & 1,18 & -95.166 & 0,16 & 2,00 & -302 & $-1,18$ & 178 & 1,38 & -208 & $-1,18$ & 178 \\
\hline $3 m-6 m$ & 1,48 & -160.009 & 0,36 & 2,00 & -1.152 & $-1,48$ & 851 & 1,33 & -766 & $-1,48$ & 851 \\
\hline $6 \mathrm{~m}-1 \mathrm{a}$ & 1,79 & -118.378 & 0,71 & 2,00 & -1.681 & $-1,79$ & 1.505 & 1,24 & -1.045 & $-1,79$ & 1.505 \\
\hline $1 a-2 a$ & 1,37 & -104.119 & 1,38 & 2,00 & -2.874 & $-1,37$ & 1.967 & 1,19 & -1.713 & $-1,37$ & 1.967 \\
\hline $\mathbf{2 a}-\mathbf{3 a}$ & 1,35 & -104.119 & 2,25 & 2,00 & -4.685 & $-1,35$ & 3.158 & 1,10 & -2.574 & $-1,35$ & 3.158 \\
\hline $3 a-4 a$ & 1,45 & -104.119 & 3,07 & 2,00 & -6.393 & $-1,45$ & 4.643 & 1,03 & -3.286 & $-1,45$ & 4.643 \\
\hline $4 a-5 a$ & 1,63 & -104.119 & 3,85 & 2,00 & -8.017 & $-1,63$ & 6.520 & 0,98 & -3.921 & $-1,63$ & 6.520 \\
\hline $5 a-7 a$ & 1,91 & 60.227 & 5,08 & 2,00 & 6.119 & $-1,91$ & -5.850 & 0,91 & 2.775 & $-1,91$ & -5.850 \\
\hline $7 \mathbf{a}-10 a$ & 2,24 & 90.341 & 6,63 & 2,00 & 11.979 & $-2,00$ & -11.979 & 0,82 & 4.900 & $-1,50$ & -8.984 \\
\hline $10 a-15 a$ & 2,57 & 11.721 & 8,92 & 2,00 & 2.091 & $-2,00$ & -2.091 & 0,76 & 795 & $-1,19$ & -1.240 \\
\hline $15 a-20 a$ & 2,68 & 11.721 & 11,21 & 2,00 & 2.628 & $-2,00$ & -2.628 & 0,75 & 982 & $-1,06$ & -1.398 \\
\hline Oltre i 20a & 2,65 & 11.721 & 13,01 & 2,00 & 3.050 & $-2,00$ & -3.050 & 0,75 & 1.150 & $-1,15$ & -1.749 \\
\hline$\Delta \mathbf{V E}$ & & & & & 725 & & -6.762 & & -2.934 & & -386 \\
\hline $\mathbf{I R}=\Delta V \mathbf{V E} / \mathbf{F P}$ & & & & & 0,34 & & $-3,15$ & & $-1,37$ & & $-0,18$ \\
\hline
\end{tabular}

Fonte: Elaborazioni dell'Autore su dati di bilancio al 31/12/2011 e dati Datastream.

\section{L'impatto del portafoglio titoli sulla complessiva esposizione al rischio}

Il portafoglio titoli della banca, è, per ipotesi, esposto a sole variazioni in aumento dei tassi di interesse in quanto composto da poste di bilancio che danno luogo esclusivamente a posizioni nette positive. La restante parte del portafoglio bancario, nel proseguo detta banca commerciale, rappresentata dalla tradizionale attività di impiego e di raccolta può essere, invece, esposta a variazioni in aumento o in diminuzione dei tassi di interesse a seconda della specifica struttura per scadenza delle posizioni nette ad essa associata. Nello specifico, il portafoglio titoli, nella prospettiva del rischio di tasso di interesse del portafoglio bancario, è composto da tutti i titoli iscritti nei vari portafogli contabili ad eccezione di quello di negoziazione. La banca commerciale comprende, invece, le varie tipologie di prestiti erogati e le varie forme tecniche di raccolta emesse comprensive dei prestiti obbligazionari. La somma algebrica delle strutture per scadenza delle posizioni nette associate alle due suddette linee di business (portafoglio titoli e banca commerciale) conduce, per costruzione, alla struttura per scadenza delle posizioni nette relativa all'intero portafoglio bancario, nel proseguo della trattazione indicato come intera banca. L'interazione tra il portafoglio titoli e la banca commerciale può impattare sulla complessiva esposizione al rischio dell'intera banca in differenti modalità. Nel proseguo si descrivono le varie ipotesi che possono presentarsi nell'ambito dell'operatività bancaria. Per quel che concerne i dati utilizzati ai fini dell'analisi si rimanda a quanto detto nel precedente paragrafo 4 . Le banche di seguito considerate sono parte del campione analizzato nel proseguo dell'analisi e sono state scelte in quanto rappresentative delle differenti ipotesi individuate.

\section{Ipotesi 1: l'intera banca è esposta a variazioni in aumento dei tassi di interesse cosi come la banca commerciale e il} portafoglio titoli.

In tale ipotesi le due linee di business contribuiscono in modo analogo ma con differenti intensità alla determinazione della tipologia di esposizione al rischio dell'intera banca. La seguente tabella 5 riporta il caso in esame. Dalla seconda alla quinta colonna sono riportati il livello dei key-rates alla data di valutazione e le strutture per scadenza delle posizioni nette associate all'intera banca, alla banca commerciale e al portafoglio titoli. Successivamente sono indicati i livelli di duration associati alle varie fasce temporali e gli scenari di variazione dei tassi di interesse adottati nell'ambito delle due metodologie regolamentari ai fini della stima dell'esposizione al rischio. Le ultime sei colonne riportano il calcolo delle relative posizioni nette ponderate per l'intera banca, la banca commerciale e il portafoglio titoli. In tale caso l'intera banca è esposta a variazioni in aumento dei tassi di interesse nell'ipotesi di adozione sia dello scenario parallelo dei +200 punti base sia del $99^{\circ}$ percentile con un valore dell'indicatore complessivo di rischio che si attesta, rispettivamente, al $15,54 \%$ e al $4,97 \%$. La banca commerciale e l'insieme dei titoli di proprietà risultano entrambi esposti a variazioni in aumento dei tassi di interesse presentando un livello dell'indicatore di rischio pari, rispettivamente, al 13,25\% e al 2,29\% nell'ipotesi di applicazione dello scenario parallelo dei +200 punti base e al 3,88\% e 1,09\% nell'ipotesi, invece, di utilizzo del metodo dei percentili. 
Tabella 5. Intera banca e banca commerciale esposte a variazioni in aumento dei tassi di interesse

(PN, $\triangle \mathrm{PN}$ e $\triangle \mathrm{VE}$ in migliaia di euro - key-rates e IR in valori percentuali)

\begin{tabular}{|c|c|c|c|c|c|c|c|c|c|c|c|c|c|}
\hline \multirow{2}{*}{ Fasce temporali } & \multirow{2}{*}{ key-rates } & \multirow{2}{*}{$\begin{array}{l}\text { Intera } \\
\text { banca }\end{array}$} & \multirow{2}{*}{$\begin{array}{c}\text { Banca } \\
\text { commerciale } \\
\text { PN (b) }\end{array}$} & \multirow{2}{*}{$\begin{array}{c}\text { Portafoglio } \\
\text { titoli } \\
\text { PN (c) }\end{array}$} & \multirow{2}{*}{$\begin{array}{l}\text { Duration } \\
\text { (d) }\end{array}$} & \multirow{2}{*}{$\begin{array}{l}2,00 \\
\text { (e) }\end{array}$} & \multirow{2}{*}{$\begin{array}{c}9^{\circ} \\
(f)\end{array}$} & \multirow{2}{*}{$\begin{array}{c}\begin{array}{c}\text { Intera } \\
\text { banca }\end{array} \\
\text { PNP } \\
(\mathbf{a})^{*}(\mathbf{d})^{*}(\mathbf{e})\end{array}$} & \multirow{2}{*}{$\begin{array}{c}\begin{array}{c}\text { Banca } \\
\text { commerciale }\end{array} \\
\begin{array}{c}\text { PNP } \\
(\mathbf{b})^{*}(\mathbf{d})^{*}(\mathbf{e})\end{array}\end{array}$} & \multirow{2}{*}{$\begin{array}{c}\begin{array}{c}\text { Portafogli } \\
\text { titoli }\end{array} \\
\begin{array}{c}\text { PNP } \\
(\mathbf{c})^{*}(\mathbf{d})^{*}(\mathrm{e})\end{array}\end{array}$} & \multirow{2}{*}{$\begin{array}{c}\begin{array}{c}\text { Intera } \\
\text { banca }\end{array} \\
\begin{array}{c}\text { PNP } \\
(\mathbf{a})^{*}(\mathbf{d})^{*}(\mathbf{f})\end{array}\end{array}$} & \multirow{2}{*}{$\begin{array}{c}\begin{array}{c}\text { Banca } \\
\text { commerciale }\end{array} \\
\begin{array}{c}\text { PNP } \\
(\mathbf{b})^{*}(\mathbf{d}) *(\mathbf{f})\end{array}\end{array}$} & \multirow{2}{*}{$\begin{array}{c}\begin{array}{c}\text { Portafoglio } \\
\text { titoli }\end{array} \\
\begin{array}{c}\text { PNP } \\
(\mathbf{c})^{*}(\mathbf{d})^{*}(\mathbf{f})\end{array}\end{array}$} \\
\hline & & & & & & & & & & & & & \\
\hline A vista & 0,63 & -874.662 & -876.137 & 1.475 & 0,00 & 2,00 & 1,31 & 0 & 0 & 0 & 0 & 0 & 0 \\
\hline fino a $1 \mathrm{~m}$ & 0,76 & 478.580 & 470.823 & 7.757 & 0,04 & 2,00 & 1,24 & 380 & 374 & 6 & 236 & 232 & 4 \\
\hline $1 \mathrm{~m}-3 \mathrm{~m}$ & 1,18 & 957.160 & 941.646 & 15.513 & 0,16 & 2,00 & 1,38 & 3.039 & 2.989 & 49 & 2.090 & 2.057 & 34 \\
\hline $3 m-6 m$ & 1,48 & -120.065 & -120.065 & 0 & 0,36 & 2,00 & 1,33 & -864 & -864 & 0 & -574 & -574 & 0 \\
\hline $6 \mathrm{~m}-1 \mathrm{a}$ & 1,79 & -135.335 & -135.335 & 0 & 0,71 & 2,00 & 1,24 & -1.922 & -1.922 & 0 & -1.194 & -1.194 & 0 \\
\hline $1 \mathbf{a}-2 \mathbf{a}$ & 1,37 & -207.516 & -233.143 & 25.627 & 1,38 & 2,00 & 1,19 & -5.727 & -6.435 & 707 & -3.414 & -3.836 & 422 \\
\hline $\mathbf{2 a}-\mathbf{3 a}$ & 1,35 & -207.516 & -233.143 & 25.627 & 2,25 & 2,00 & 1,10 & -9.338 & -10.491 & 1.153 & -5.131 & -5.765 & 634 \\
\hline $3 a-4 a$ & 1,45 & -207.516 & -233.143 & 25.627 & 3,07 & 2,00 & 1,03 & -12.741 & -14.315 & 1.573 & -6.549 & -7.358 & 809 \\
\hline $4 a-5 a$ & 1,63 & -207.516 & -233.143 & 25.627 & 3,85 & 2,00 & 0,98 & -15.979 & -17.952 & 1.973 & -7.815 & -8.780 & 965 \\
\hline $5 \mathbf{a}-7 \mathbf{a}$ & 1,91 & 154.732 & 142.139 & 12.593 & 5,08 & 2,00 & 0,91 & 15.721 & 14.441 & 1.279 & 7.129 & 6.549 & 580 \\
\hline $7 a-10 a$ & 2,24 & 232.097 & 213.208 & 18.889 & 6,63 & 2,00 & 0,82 & 30.776 & 28.271 & 2.505 & 12.589 & 11.565 & 1.025 \\
\hline $10 a-15 a$ & 2,57 & 98.111 & 96.893 & 1.219 & 8,92 & 2,00 & 0,76 & 17.503 & 17.286 & 217 & 6.654 & 6.571 & 83 \\
\hline $15 a-20 a$ & 2,68 & 98.111 & 96.893 & 1.219 & 11,21 & 2,00 & 0,75 & 21.997 & 21.723 & 273 & 8.221 & 8.118 & 102 \\
\hline Oltre 20a & 2,65 & 98.111 & 96.893 & 1.219 & 13,01 & 2,00 & 0,75 & 25.529 & 25.211 & 317 & 9.627 & 9.507 & 120 \\
\hline$\Delta V E$ & & & & & & & & 68.371 & 58.317 & 10.055 & 21.867 & 17.091 & 4.776 \\
\hline $\mathbf{I R}=\Delta \mathrm{VE} / \mathrm{FP}$ & & & & & & & & 15,54 & 13,25 & 2,29 & 4,97 & 3,88 & 1,09 \\
\hline
\end{tabular}

Fonte: Elaborazioni dell'Autore su dati di bilancio al 31/12/2011 e dati Datastream.

Ipotesi 2: l'intera banca è esposta a variazioni in aumento dei tassi di interesse cosi come il portafoglio titoli. La banca commerciale è, invece, esposta a variazioni in diminuzione dei tassi di interesse.

In tale ipotesi il portafoglio titoli determina la tipologia di esposizione al rischio dell'intera banca. La banca commerciale è, invece, esposta a variazioni in diminuzione dei tassi di interesse. L'applicazione alla banca commerciale dello specifico scenario di variazioni positive su base annua dei key-rates a cui è esposta l'intera banca determina, infatti, un incremento di valore economico dei fondi propri che attenua la riduzione di valore economico determinata dal portafoglio titoli. La seguente tabella 6 riporta il caso appena descritto di una banca esposta a variazioni in aumento dei tassi di interesse nell'ipotesi di adozione sia dello scenario parallelo dei +200 punti base sia del $99^{\circ}$ percentile con un valore dell'indicatore complessivo di rischio che si attesta, rispettivamente, all' $8,67 \%$ e al 2,00\%. La riduzione di valore economico dei fondi propri riconducibile al portafoglio titoli, che, in corrispondenza delle due suddette metodologie di misurazione, conduce ad un livello dell'indicatore di rischio pari, rispettivamente, all' $11,86 \%$ e 5,22\% è attenuata dall'incremento di valore economico ottenuto dalla banca commerciale che, in termini di livello dell'indicatore di rischio, risulta pari, rispettivamente, al $-3,19 \%$ e al $3,22 \%$. 


\begin{tabular}{|c|c|c|c|c|c|c|c|c|c|c|c|c|c|}
\hline \multirow{2}{*}{ Fasce temporali } & \multirow{2}{*}{$\begin{array}{l}\text { key- } \\
\text { rates }\end{array}$} & $\begin{array}{l}\text { Intera } \\
\text { banca }\end{array}$ & $\begin{array}{c}\text { Banca } \\
\text { commerciale }\end{array}$ & $\begin{array}{c}\text { Portafoglio } \\
\text { titoli }\end{array}$ & \multirow{2}{*}{$\begin{array}{l}\text { Duration } \\
\text { (d) }\end{array}$} & \multirow{2}{*}{$\begin{array}{l}2,00 \\
\text { (e) }\end{array}$} & \multirow{2}{*}{$\begin{array}{l}\mathbf{9 9}^{\circ} \\
\text { (f) }\end{array}$} & $\begin{array}{l}\text { Intera } \\
\text { banca }\end{array}$ & $\begin{array}{c}\text { Banca } \\
\text { commerciale }\end{array}$ & $\begin{array}{c}\text { Portafogli } \\
\text { titoli }\end{array}$ & $\begin{array}{l}\text { Intera } \\
\text { banca }\end{array}$ & $\begin{array}{c}\text { Banca } \\
\text { commerciale }\end{array}$ & $\begin{array}{l}\text { Portafoglio } \\
\text { titolii }\end{array}$ \\
\hline & & & $\mathbf{P N}$ (b) & & & & & $\begin{array}{c}\text { PNP } \\
(\text { a) })^{*}(\mathbf{d})^{*}(\mathbf{e})\end{array}$ & $\underset{\left.(\mathbf{P N})^{*(\mathbf{d}}\right)^{*}(\mathbf{e})}{\mathbf{p}}$ & $\begin{array}{c}\text { PNP } \\
(\mathbf{c}) *(\mathbf{d})^{*}(\mathbf{e})\end{array}$ & $\begin{array}{c}\text { PNP } \\
(\mathbf{a}) *(\mathbf{d}) *(\mathbf{f})\end{array}$ & $\underset{(\mathbf{P N P}) *(\mathbf{d}) *(\mathbf{f})}{ }$ & $\underset{(\mathbf{c})^{*}(\mathbf{d})^{* *(\mathbf{f})}}{\text {. }}$ \\
\hline A vista & 0,63 & -14.380 & -29.085 & 14.705 & 0,00 & 2,00 & 1,31 & 0 & 0 & 0 & 0 & 0 & 0 \\
\hline fino a 1m & 0,76 & 35.406 & 29.414 & 5.992 & 0,04 & 2,00 & 1,24 & 28 & 23 & 5 & 17 & 14 & 3 \\
\hline $1 m-3 m$ & 1,18 & 70.813 & 58.828 & 11.985 & 0,16 & 2,00 & 1,38 & 225 & 187 & 38 & 155 & 128 & 26 \\
\hline $3 m-6 m$ & 1,48 & -10.998 & -16.797 & 5.799 & 0,36 & 2,00 & 1,33 & -79 & -121 & 42 & -53 & -80 & 28 \\
\hline $6 \mathrm{~m}-1 \mathrm{a}$ & 1,79 & -26.755 & -26.755 & 0 & 0,71 & 2,00 & 1,24 & -380 & -380 & 0 & -236 & -236 & 0 \\
\hline $1 a-2 a$ & 1,37 & -44.413 & -50.294 & 5.881 & 1,38 & 2,00 & 1,19 & -1.226 & -1.388 & 162 & -731 & -827 & 97 \\
\hline $2 a-3 a$ & 1,35 & -44.413 & -50.294 & 5.881 & 2,25 & 2,00 & 1,10 & -1.999 & -2.263 & 265 & -1.098 & -1.244 & 145 \\
\hline $3 a-4 a$ & 1,45 & -44.413 & -50.294 & 5.881 & 3,07 & 2,00 & 1,03 & -2.727 & -3.088 & 361 & -1.402 & -1.587 & 186 \\
\hline $4 a-5 a$ & 1,63 & -44.413 & -50.294 & 5.881 & 3,85 & 2,00 & 0,98 & -3.420 & -3.873 & 453 & -1.673 & -1.894 & 221 \\
\hline $5 a-7 a$ & 1,91 & 33.650 & 9.207 & 24.443 & 5,08 & 2,00 & 0,91 & 3.419 & 935 & 2.483 & 1.550 & 424 & 1.126 \\
\hline $7 a-10 a$ & 2,24 & 50.474 & 13.810 & 36.664 & 6,63 & 2,00 & 0,82 & 6.693 & 1.831 & 4.862 & 2.738 & 749 & 1.989 \\
\hline 10a-15a & 2,57 & 8.827 & 8.732 & 94 & 8,92 & 2,00 & 0,76 & 1.575 & 1.558 & 17 & 599 & 592 & 6 \\
\hline $15 a-20 a$ & 2,68 & 8.827 & 8.732 & 94 & 11,21 & 2,00 & 0,75 & 1.979 & 1.958 & 21 & 740 & 732 & 8 \\
\hline Oltre 20a & 2,65 & 8.827 & 8.732 & 94 & 13,01 & 2,00 & 0,75 & 2.297 & 2.272 & 25 & 866 & 857 & 9 \\
\hline$\Delta V E$ & & & & & & & & 6.385 & -2.348 & 8.733 & 1.473 & -2.372 & 3.845 \\
\hline $\mathbf{I R}=\Delta V \mathbf{E} / \mathbf{F P}$ & & & & & & & & 8,67 & $-3,19$ & 11,86 & 2,00 & $-3,22$ & 5,22 \\
\hline
\end{tabular}

Fonte: Elaborazioni dell'Autore su dati di bilancio al 31/12/2011 e dati Datastream.

Ipotesi3: l'intera banca è esposta a variazioni in diminuzione dei tassi di interesse cosi come la banca commerciale Il portafoglio titoli è esposto a variazioni in aumento dei tassi di interesse

In tale ipotesi è la banca commerciale che determina la tipologia di esposizione al rischio dell'intera banca. L'applicazione all'insieme dei titoli di proprietà dello specifico scenario di variazioni negative su base annua dei key-rates a cui è esposta l'intera banca determina, infatti, un incremento di valore economico dei fondi propri che attenua la riduzione di valore economico riconducibile alla banca commerciale ${ }^{6}$. La seguente tabella 7 riporta il caso appena descritto di una banca esposta a variazioni in diminuzione dei tassi di interesse nell'ipotesi di adozione sia dello scenario parallelo dei -200 punti base sia del $1^{\circ}$ percentile con un valore dell'indicatore di rischio che si attesta, rispettivamente, al 7,25\% e all' $8,35 \%$. La riduzione di valore economico dei fondi propri riconducibile alla banca commerciale che, in corrispondenza delle due suddette metodologie di misurazione, conduce ad un livello dell'indicatore di rischio pari, rispettivamente, al 10,47\% e all' $11,14 \%$ è attenuata dall'incremento di valore economico ottenuto sul portafoglio titoli che, in termini di livello dell'indicatore di rischio, risulta pari, rispettivamente, al $-3,22 \%$ e al $-2,79 \%$.

\footnotetext{
${ }^{6}$ Quanto detto si verifica nell'ipotesi in cui il livello dei key-rates vigente alla data di valutazione è maggiore di zero. In tale ipotesi gli scenari dei -200 punti base e del $1^{\circ}$ percentile sono caratterizzati da variazioni negative su tutte le fasce temporali della matrice per scadenza e per data di riprezzamento. Nell'ipotesi in cui, invece, come effettivamente accaduto negli ultimi anni oggetto di analisi, si registrano su specifiche fasce temporali, alla data di valutazione livelli negativi dei key-rates i due suddetti scenari sono caratterizzati, su tali fasce temporali, da variazioni positive. Di conseguenza, le posizioni nette ponderate osservate sul portafoglio titoli conducono ad un decremento del valore economico dei fondi propri. È quindi possibile, in tale caso, che una banca esposta a variazioni in diminuzione dei tassi di interesse presenti una riduzione di valore economico sia in corrispondenza della banca commerciale, sia del portafoglio titoli.
} 


\begin{tabular}{|c|c|c|c|c|c|c|c|c|c|c|c|c|c|}
\hline \multirow{2}{*}{ Fasce temporali } & \multirow{2}{*}{$\begin{array}{l}\text { key- } \\
\text { rates }\end{array}$} & \multirow{2}{*}{$\begin{array}{c}\begin{array}{c}\text { Portafoglio } \\
\text { bancario }\end{array} \\
\text { PN (a) }\end{array}$} & \multirow{2}{*}{$\begin{array}{c}\begin{array}{c}\text { Banca } \\
\text { commerciale }\end{array} \\
\text { PN (b) }\end{array}$} & \multirow{2}{*}{$\begin{array}{c}\begin{array}{c}\text { Portafoglio } \\
\text { titoli }\end{array} \\
\text { PN (c) }\end{array}$} & \multirow{2}{*}{$\begin{array}{c}\text { Duration } \\
\text { (d) }\end{array}$} & \multirow{2}{*}{$\begin{array}{c}-2,00 \\
(\mathrm{e})\end{array}$} & \multirow{2}{*}{$\begin{array}{l}1^{\circ} \\
\text { (f) }\end{array}$} & \multirow{2}{*}{$\begin{array}{c}\begin{array}{c}\text { Intera } \\
\text { banca }\end{array} \\
\text { PNP } \\
(\mathbf{a})^{*}(\mathbf{d}) *(\mathbf{e})\end{array}$} & \multirow{2}{*}{$\begin{array}{c}\begin{array}{c}\text { Banca } \\
\text { commerciale }\end{array} \\
\begin{array}{c}\text { PNP } \\
(\mathbf{b}) *(\mathbf{d}) *(\mathbf{e})\end{array}\end{array}$} & \multirow{2}{*}{$\begin{array}{c}\begin{array}{c}\text { Portafogli } \\
\text { titoli }\end{array} \\
\begin{array}{c}\text { PNP } \\
(\mathbf{c}) *(\mathbf{d}) *(e)\end{array}\end{array}$} & \multirow{2}{*}{$\begin{array}{c}\begin{array}{c}\text { Intera } \\
\text { banca }\end{array} \\
\text { PNP } \\
(\mathbf{a}) *(\mathbf{d}) *(\mathbf{f})\end{array}$} & \multirow{2}{*}{$\begin{array}{c}\begin{array}{c}\text { Banca } \\
\text { commerciale }\end{array} \\
\begin{array}{c}\text { PNP } \\
(\mathbf{b})^{*}(\mathbf{d}) *(\mathbf{f})\end{array}\end{array}$} & \multirow{2}{*}{$\begin{array}{c}\begin{array}{c}\text { Portafogli } \\
\text { titoli }\end{array} \\
\text { PNP } \\
(\mathbf{c})^{*}(\mathbf{d})^{*}(\mathbf{f})\end{array}$} \\
\hline & & & & & & & & & & & & & \\
\hline A vista & 0,63 & 263.581 & 262.765 & 816 & 0,00 & $-0,63$ & $-0,63$ & 0 & 0 & 0 & 0 & 0 & 0 \\
\hline fino a $1 \mathrm{~m}$ & 0,76 & 509.892 & 503.425 & 6.467 & 0,04 & $-0,76$ & $-0,76$ & -155 & -153 & -2 & -155 & -153 & -2 \\
\hline $1 \mathrm{~m}-3 \mathrm{~m}$ & 1,18 & 1.019 .784 & 1.006 .849 & 12.935 & 0,16 & $-1,18$ & $-1,18$ & -1.907 & -1.883 & -24 & -1.907 & -1.883 & -24 \\
\hline $3 m-6 m$ & 1,48 & -176.988 & -177.388 & 400 & 0,36 & $-1,48$ & $-1,48$ & 941 & 943 & -2 & 941 & 943 & -2 \\
\hline $6 \mathrm{~m}-1 \mathrm{a}$ & 1,79 & -220.329 & -249.812 & 29.483 & 0,71 & $-1,79$ & $-1,79$ & 2.802 & 3.177 & -375 & 2.802 & 3.177 & -375 \\
\hline $1 a-2 a$ & 1,37 & -290.998 & -340.355 & 49.358 & 1,38 & $-1,37$ & $-1,37$ & 5.497 & 6.429 & -932 & 5.497 & 6.429 & -932 \\
\hline $2 a-3 a$ & 1,35 & -290.998 & -340.355 & 49.358 & 2,25 & $-1,35$ & $-1,35$ & 8.826 & 10.323 & -1.497 & 8.826 & 10.323 & -1.497 \\
\hline $3 a-4 a$ & 1,45 & -290.998 & -340.355 & 49.358 & 3,07 & $-1,45$ & $-1,45$ & 12.976 & 15.177 & -2.201 & 12.976 & 15.177 & -2.201 \\
\hline $4 a-5 a$ & 1,63 & -290.998 & -340.355 & 49.358 & 3,85 & $-1,63$ & $-1,63$ & 18.222 & 21.313 & -3.091 & 18.222 & 21.313 & -3.091 \\
\hline $5 a-7 a$ & 1,91 & 19.855 & 9.936 & 9.919 & 5,08 & $-1,91$ & $-1,91$ & -1.928 & -965 & -963 & -1.928 & -965 & -963 \\
\hline $7 a-10 a$ & 2,24 & 29.782 & 14.904 & 14.878 & 6,63 & $-2,00$ & $-1,50$ & -3.949 & -1.976 & -1.973 & -2.962 & -1.482 & -1.480 \\
\hline 10a-15a & 2,57 & 13.600 & 8.624 & 4.976 & 8,92 & $-2,00$ & $-1,19$ & -2.426 & -1.539 & -888 & -1.439 & -913 & -527 \\
\hline $15 a-20 a$ & 2,68 & 13.600 & 8.624 & 4.976 & 11,21 & $-2,00$ & $-1,06$ & -3.049 & -1.934 & -1.116 & -1.622 & -1.029 & -593 \\
\hline Oltre 20a & 2,65 & 13.600 & 8.624 & 4.976 & 13,01 & $-2,00$ & $-1,15$ & -3.539 & -2.244 & -1.295 & -2.029 & -1.287 & -742 \\
\hline$\Delta V E$ & & & & & & & & 32.310 & 46.669 & -14.358 & 37.221 & 49.651 & -12.430 \\
\hline $\mathbf{I R}=\Delta V \mathbf{V E} / \mathbf{F P}$ & & & & & & & & 7,25 & 10,47 & $-3,22$ & 8,35 & 11,14 & $-2,79$ \\
\hline
\end{tabular}

Fonte: Elaborazioni dell'Autore su dati di bilancio al 31/12/2011 e dati Datastream.

\section{Ipotesi 4: la banca è neutrale al rischio}

In tale ipotesi è interessante analizzare cosa accade in corrispondenza dei due scenari di variazioni su base annua positivi e negativi dei key-rates utilizzati ai fini della stima dell'esposizione al rischio nell'ambito delle due metodologie regolamentari. In corrispondenza dello scenario caratterizzato da variazioni positive su base annua dei key-rates (scenario di +200 punti base e $99^{\circ}$ percentile) la banca commerciale fa registrare necessariamente un incremento del valore economico dei fondi propri maggiore, in valore assoluto, della riduzione riconducibile al portafoglio titoli. In caso contrario, infatti, la banca non sarebbe più neutrale al rischio ma esposta a variazioni in aumento dei tassi di interesse.

In corrispondenza, invece, dello scenario caratterizzato da variazioni negative dei key-rates (scenario di -200 punti base e $1^{\circ}$ percentile) il portafoglio titoli fa rilevare un incremento di valore economico dei fondi propri che può essere determinante o meno ai fini della neutralità a seconda della tipologia di esposizione al rischio presentata dalla banca commerciale. Al riguardo possono, quindi, essere individuati due differenti casi di seguito descritti nell'ipotesi di applicazione dello scenario parallelo dei +/-200 punti base. Considerazioni analoghe possono essere ottenute per altre banche del campione a seguito dell'applicazione del metodo dei percentili.

Nel primo caso, riportato nella seguente Tabella 8 e indicato nel proseguo della trattazione come ipotesi 4a, la neutralità al rischio si verifica a prescindere dall'impatto del portafoglio titoli di proprietà. La banca commerciale è, infatti, neutrale al rischio in quanto conduce ad un incremento del valore economico dei fondi propri sia nello scenario di +200 punti base sia in quello di -200 punti base, facendo registrare un incremento di valore economico, in termini di livello dell'indicatore di rischio, pari, rispettivamente, di $-2,69 \%$ e $-0,48 \%$. Il portafoglio titoli è caratterizzato, in corrispondenza dello scenario di +200 punti base da un decremento di valore economico dei fondi propri, in termini di livello dell'indicatore di rischio, pari a 1,98\%, inferiore, in valore assoluto, all'incremento riconducibile alla banca commerciale. Nell'ipotesi, invece, di scenario di - 200 punti base il portafoglio titoli fa registrare, per costruzione, un incremento di valore economico dei fondi propri pari, in termini di livello dell'indicatore di rischio, al $-1,49 \%$, che contribuisce, unitamente alla riduzione osservata per la banca commerciale, a rendere la banca neutrale al rischio ${ }^{7}$.

\footnotetext{
${ }^{7}$ Come detto nella precedente nota 5 nell'ipotesi di livelli negativi dei key-rates alla data di valutazione è possibile che l'applicazione dello scenario dei -200 punti base e del $1^{\circ}$ percentile possa condurre ad una riduzione di valore economico dei fondi propri. Nell'ipotesi $4 \mathrm{a}$ oggetto di analisi se la riduzione di valore economico dei fondi propri associato al portafoglio titoli è minore, in valore assoluto, dell'incremento registrato per la banca commerciale si rimane nell'ambito dell'ipotesi in questione. Se, invece, la riduzione del valore economico dei fondi propri associato al portafoglio titoli è maggiore, in valore assoluto, dell'incremento registrato per la banca commerciale l'intera banca sarebbe esposta a variazioni in diminuzione dei tassi di interesse.
} 
Tabella 8. Intera banca e banca commerciale neutrale al rischio.

(PN, $\triangle \mathrm{PN}$ e $\Delta \mathrm{VE}$ in migliaia di euro - key-rates e IR in valori percentuali)

\begin{tabular}{|c|c|c|c|c|c|c|c|c|c|c|c|c|c|}
\hline \multirow{2}{*}{ Fasce temporali } & \multirow{2}{*}{$\begin{array}{l}\text { key- } \\
\text { rates }\end{array}$} & $\begin{array}{l}\text { Intera } \\
\text { banca }\end{array}$ & $\begin{array}{c}\text { Banca } \\
\text { commerciale }\end{array}$ & $\begin{array}{l}\text { Portafoglio } \\
\text { titoli }\end{array}$ & \multirow{2}{*}{$\begin{array}{l}\text { Duration } \\
\text { (d) }\end{array}$} & \multirow{2}{*}{$\begin{array}{l}2,00 \\
\text { (e) }\end{array}$} & \multirow{2}{*}{$\begin{array}{c}-2,00 \\
\text { (f) }\end{array}$} & $\begin{array}{l}\text { Intera } \\
\text { banca }\end{array}$ & $\begin{array}{c}\text { Banca } \\
\text { commerciale }\end{array}$ & $\begin{array}{l}\text { Portafogli } \\
\text { titoli }\end{array}$ & $\begin{array}{l}\text { Intera } \\
\text { banca }\end{array}$ & $\begin{array}{c}\text { Banca } \\
\text { commerciale }\end{array}$ & $\begin{array}{c}\text { Portafoglio } \\
\text { titoli }\end{array}$ \\
\hline & & PN (a) & PN (b) & PN (c) & & & & $\begin{array}{c}\text { PNP } \\
(\mathrm{a})^{*}(\mathrm{~d})^{*}(\mathrm{e})\end{array}$ & $\begin{array}{c}\text { PNP } \\
(\mathrm{b})^{*}(\mathrm{~d})^{*}(\mathrm{e})\end{array}$ & $\begin{array}{c}\text { PNP } \\
(\mathrm{c})^{*}(\mathrm{~d})^{*}(\mathrm{e})\end{array}$ & $\begin{array}{c}\text { PNP } \\
(\text { a)*(d)*(f) }\end{array}$ & $\begin{array}{c}\text { PNP } \\
(\mathbf{b}) *(\mathrm{~d}) *(\mathrm{f})\end{array}$ & $\begin{array}{c}\text { PNP } \\
(\mathrm{c})^{*}(\mathrm{~d})^{*}(\mathrm{f})\end{array}$ \\
\hline A vista & 0,63 & 1.104 .114 & 1.014 .556 & 89.558 & 0,00 & 2,00 & $-0,63$ & 0 & 0 & 0 & 0 & 0 & 0 \\
\hline fino a $1 \mathrm{~m}$ & 0,76 & 132.917 & 71.372 & 61.545 & 0,04 & 2,00 & $-0,76$ & 105 & 57 & 49 & -40 & -22 & -19 \\
\hline $1 m-3 m$ & 1,18 & 265.834 & 142.744 & 123.090 & 0,16 & 2,00 & $-1,18$ & 844 & 453 & 391 & -497 & -267 & -230 \\
\hline $3 m-6 m$ & 1,48 & -343.595 & -454.446 & 110.851 & 0,36 & 2,00 & $-1,48$ & -2.474 & -3.272 & 798 & 1.826 & 2.416 & -589 \\
\hline $6 \mathrm{~m}-1 \mathrm{a}$ & 1,79 & -305.603 & -366.758 & 61.155 & 0,71 & 2,00 & $-1,79$ & -4.340 & -5.208 & 868 & 3.886 & 4.664 & -778 \\
\hline $1 a-2 a$ & 1,37 & -323.384 & -373.940 & 50.556 & 1,38 & 2,00 & $-1,37$ & -8.925 & -10.321 & 1.395 & 6.108 & 7.063 & -955 \\
\hline $2 a-3 a$ & 1,35 & -323.384 & -373.940 & 50.556 & 2,25 & 2,00 & $-1,35$ & -14.552 & -16.827 & 2.275 & 9.808 & 11.342 & -1.533 \\
\hline $3 a-4 a$ & 1,45 & -323.384 & -373.940 & 50.556 & 3,07 & 2,00 & $-1,45$ & -19.856 & -22.960 & 3.104 & 14.420 & 16.675 & -2.254 \\
\hline $4 a-5 a$ & 1,63 & -323.384 & -373.940 & 50.556 & 3,85 & 2,00 & $-1,63$ & -24.901 & -28.793 & 3.893 & 20.250 & 23.416 & -3.166 \\
\hline $5 a-7 a$ & 1,91 & 96.556 & 95.237 & 1.319 & 5,08 & 2,00 & $-1,91$ & 9.810 & 9.676 & 134 & -9.378 & -9.250 & -128 \\
\hline $7 a-10 a$ & 2,24 & 144.834 & 142.855 & 1.979 & 6,63 & 2,00 & $-2,00$ & 19.205 & 18.943 & 262 & -19.205 & -18.943 & -262 \\
\hline $10 a-15 a$ & 2,57 & 60.824 & 60.754 & 70 & 8,92 & 2,00 & $-2,00$ & 10.851 & 10.839 & 12 & -10.851 & -10.839 & -12 \\
\hline $15 a-20 a$ & 2,68 & 60.824 & 60.754 & 70 & 11,21 & 2,00 & $-2,00$ & 13.637 & 13.621 & 16 & -13.637 & -13.621 & -16 \\
\hline Oltre $20 \mathrm{a}$ & 2,65 & 60.824 & 60.754 & 70 & 13,01 & 2,00 & $-2,00$ & 15.826 & 15.808 & 18 & -15.826 & -15.808 & -18 \\
\hline$\Delta \mathrm{VE}$ & & & & & & & & -4.769 & -17.985 & 13.216 & -13.136 & -3.174 & -9.961 \\
\hline $\mathrm{IR}=\Delta \mathrm{VE} / \mathrm{FP}$ & & & & & & & & $-0,71$ & $-2,69$ & 1,98 & $-1,97$ & $-0,48$ & $-1,49$ \\
\hline
\end{tabular}

Fonte: Elaborazioni dell'Autore su dati di bilancio al 31/12/2011 e Datastream.

Nel secondo caso, riportato nella seguente Tabella 9 e indicato nel proseguo della trattazione come ipotesi $4 b$, è, invece, l'insieme del portafoglio titoli a determinare la neutralità al rischio dell'intera banca. La banca commerciale è, infatti, esposta a variazioni in diminuzione dei tassi di interesse. Nell'ipotesi di applicazione dello scenario di +200 punti base l'incremento di valore economico dei fondi propri rilevato in corrispondenza della banca commerciale, che, in termini di livello dell'indicatore di rischio, si colloca al $-3,43 \%$ è maggiore, in valore assoluto, del decremento di valore economico dei fondi propri osservato sul portafoglio titoli e pari, in termini di livello dell'indicatore di rischio, al 2,58\%. L'intera banca non risulta, quindi, esposta a variazioni in aumento dei tassi di interesse.

Nell'ipotesi, invece, di adozione dello scenario di -200 punti base, la riduzione di valore economico dei fondi propri registrata, dalla banca commerciale pari, in termini di livello dell'indicatore di rischio, all'1,92\%, è minore, in valore assoluto, dell'incremento di valore economico dei fondi propri rilevato sull'insieme dei titoli di proprietà, che si attesta al $-2,27 \%$. L'intera banca non risulta, quindi, esposta a variazioni in diminuzione dei tassi di interesse ${ }^{8}$.

${ }^{8}$ Facendo seguito a quanto riportato nelle precedenti note 5 e 6 nell'ipotesi di un decremento di valore economico dei fondi propri associato al portafoglio titoli a seguito dell'applicazione dello scenario dei -200 punti base e del $1^{\circ}$ percentile l'intera banca sarebbe esposta a variazioni in diminuzione dei tassi di interesse. 


\begin{tabular}{|c|c|c|c|c|c|c|c|c|c|c|c|c|c|}
\hline Fasce temporali & $\begin{array}{l}\text { key- } \\
\text { rates }\end{array}$ & $\begin{array}{l}\text { Intera } \\
\text { banca }\end{array}$ & $\begin{array}{c}\begin{array}{c}\text { Banca } \\
\text { commerciale }\end{array} \\
\text { PN (b) }\end{array}$ & $\begin{array}{l}\begin{array}{c}\text { Portafoglio } \\
\text { titoli }\end{array} \\
\text { PN (c) }\end{array}$ & $\begin{array}{l}\text { Duration } \\
\text { (d) }\end{array}$ & $\begin{array}{c}2,00 \\
\text { (e) }\end{array}$ & $\begin{array}{c}-2,00 \\
\text { (f) }\end{array}$ & $\begin{array}{l}\text { Intera } \\
\text { banca } \\
\\
\text { PNP } \\
\text { (a) })^{*}(\mathbf{d})^{*}(\mathbf{e})\end{array}$ & $\begin{array}{c}\begin{array}{c}\text { Banca } \\
\text { commerciale }\end{array} \\
\text { PNP } \\
(\mathbf{b}) *(\mathbf{d}) *(\mathbf{e})\end{array}$ & $\begin{array}{l}\text { Portafogli } \\
\text { titoli } \\
\text { PNP } \\
\text { (c) }{ }^{*}(\mathbf{d})^{*}(\mathbf{e})\end{array}$ & $\begin{array}{c}\text { Intera } \\
\text { banca } \\
\\
\text { PNP } \\
\text { (a) } *(\text { d) } *(\mathbf{f})\end{array}$ & $\begin{array}{c}\begin{array}{c}\text { Banca } \\
\text { commerciale }\end{array} \\
\text { PNP } \\
(\mathbf{b})^{*}(\mathbf{d})^{*} *(\mathbf{f})\end{array}$ & $\begin{array}{l}\begin{array}{c}\text { Portafoglio } \\
\text { titoli }\end{array} \\
\text { PNP } \\
(\mathbf{c}) *(\mathbf{d}) *(\mathbf{f})\end{array}$ \\
\hline A vista & 0,63 & 56.046 & 52.209 & 3.837 & 0,00 & 2,00 & $-0,63$ & 0 & 0 & 0 & 0 & 0 & 0 \\
\hline fino a $1 \mathrm{~m}$ & 0,76 & 151.484 & 110.356 & 41.128 & 0,04 & 2,00 & $-0,76$ & 120 & 88 & 33 & -46 & -33 & -12 \\
\hline $1 \mathrm{~m}-3 \mathrm{~m}$ & 1,18 & 302.969 & 220.712 & 82.257 & 0,16 & 2,00 & $-1,18$ & 962 & 701 & 261 & -567 & -413 & -154 \\
\hline $3 m-6 m$ & 1,48 & 30.592 & -57.310 & 87.902 & 0,36 & 2,00 & $-1,48$ & 220 & -413 & 633 & -163 & 305 & -467 \\
\hline $6 m-1 a$ & 1,79 & -9.240 & -38.228 & 28.988 & 0,71 & 2,00 & $-1,79$ & -131 & -543 & 412 & 117 & 486 & -369 \\
\hline 1a-2a & 1,37 & -109.735 & -128.013 & 18.278 & 1,38 & 2,00 & $-1,37$ & -3.029 & -3.533 & 504 & 2.073 & 2.418 & -345 \\
\hline $2 a-3 a$ & 1,35 & -109.735 & -128.013 & 18.278 & 2,25 & 2,00 & $-1,35$ & -4.938 & -5.761 & 823 & 3.328 & 3.883 & -554 \\
\hline $3 a-4 a$ & 1,45 & -109.735 & -128.013 & 18.278 & 3,07 & 2,00 & $-1,45$ & -6.738 & -7.860 & 1.122 & 4.893 & 5.708 & -815 \\
\hline $4 a-5 a$ & 1,63 & -109.735 & -128.013 & 18.278 & 3,85 & 2,00 & $-1,63$ & -8.450 & -9.857 & 1.407 & 6.872 & 8.016 & -1.145 \\
\hline $5 a-7 a$ & 1,91 & 29.958 & 21.996 & 7.962 & 5,08 & 2,00 & $-1,91$ & 3.044 & 2.235 & 809 & -2.910 & -2.137 & -773 \\
\hline $7 a-10 a$ & 2,24 & 44.938 & 32.995 & 11.943 & 6,63 & 2,00 & $-2,00$ & 5.959 & 4.375 & 1.584 & -5.959 & -4.375 & -1.584 \\
\hline 10a-15a & 2,57 & 13.902 & 7.987 & 5.915 & 8,92 & 2,00 & $-2,00$ & 2.480 & 1.425 & 1.055 & -2.480 & -1.425 & -1.055 \\
\hline $15 a-20 a$ & 2,68 & 13.902 & 7.987 & 5.915 & 11,21 & 2,00 & $-2,00$ & 3.117 & 1.791 & 1.326 & -3.117 & -1.791 & -1.326 \\
\hline Oltre 20a & 2,65 & 13.902 & 7.987 & 5.915 & 13,01 & 2,00 & $-2,00$ & 3.617 & 2.078 & 1.539 & -3.617 & -2.078 & -1.539 \\
\hline$\Delta V E$ & & & & & & & & -3.766 & -15.274 & 11.508 & -1.575 & 8.564 & -10.139 \\
\hline $\mathbf{I R}=\Delta \mathbf{V E} / \mathbf{F P}$ & & & & & & & & $-0,84$ & $-3,43$ & 2,58 & $-0,35$ & 1,92 & $-2,27$ \\
\hline
\end{tabular}

Fonte: Elaborazioni dell'Autore su dati di bilancio al 31/12/2011 e dati Datastream.

\section{Le evidenze empiriche nel periodo 2006-2015}

La stima dell'indicatore di rischio regolamentare è stata effettuata su un campione di 40 banche italiane nel periodo 2006-2015 sulla base dei dati di bilancio pubblicati al 31/12 di ogni anno. Si rimanda a quanto detto nel precedente paragrafo 4 per un maggiore dettaglio sui dati utilizzati ai fini dell'analisi. Il campione è costituito dalle principali banche commerciali italiane per le quali sono disponibili i dati in questione nell'intero periodo oggetto di analisi. Sono state escluse dal campione le banche di credito cooperativo. Le stime sono state effettuate utilizzando nell'intero orizzonte temporale considerato il criterio di distribuzione dei depositi introdotto nel 2011 che prevede la ripartizione di tali poste di bilancio nelle fasce temporali fino a 5 anni cosi come descritto nel precedente paragrafo 3. La successiva Tabella 10 mostra la composizione media in termini percentuali delle attività e delle passività per cassa sul totale delle stesse per l'intero campione considerato. L'analisi dei dati evidenzia che nel periodo oggetto di analisi il portafoglio crediti è stato pari in media all' $84,47 \%$ del totale delle attività per cassa con una componente a breve termine (fasce temporali a vista e fino ad 1 anno), del 72,03\% rappresentata dall'intero debito residuo dei mutui a tasso variabile e dalle quote capitali in scadenza in tale orizzonte temporale dei mutui a tasso fisso. La restante parte del 15,54\% è costituita da titoli iscritti nel portafoglio bancario. Essi hanno mostrato una dinamica crescente nel periodo oggetto di analisi passando dal 7,71\% nel 2006 al $25,83 \%$ nel 2015 con la componente a medio e lungo termine (oltre 1 anno) che ha fatto registrare un aumento dal 2,26\% al 10,33\%. I depositi a vista rappresentano in media il 44,75\% delle passività per cassa. I titoli di debito emessi rappresentano, invece, il 30,72\% delle passività per cassa di cui il 29,85\% è a breve e medio termine. La restante parte (Altra raccolta) pari al 24,53\% è costituita, essenzialmente, da raccolta a vista presso il mercato interbancario, dalle operazioni di rifinanziamento presso la Banca Centrale Europea (BCE) e da forme tecniche vincolate a scadenza sottoscritte da differenti tipologie di clientela. Si registra in tale aggregato di bilancio il forte peso della componente con scadenza inferiore ad 1 anno che presenta un valore medio di periodo del 13,37\% e livelli elevati negli ultimi anni. Nel 2013 si è registrata, infatti, una quota del 22,29\% che è, successivamente, scesa al 18,50\% nel 2014 e al 16,73\% nel 2015. 
Tabella 10. Composizione percentuale dell'attivo e del passivo bancario

(valori percentuali sul totale delle attività e delle passività per cassa)

\begin{tabular}{|c|c|c|c|c|c|c|c|c|c|c|c|}
\hline Attività per cassa & 2006 & 2007 & 2008 & 2009 & 2010 & 2011 & 2012 & 2013 & 2014 & 2015 & media \\
\hline Titoli con scadenza inferiore a 1 anno & 5,45 & 6,76 & 8,23 & 8,66 & 9,16 & 8,03 & 10,61 & 13,36 & 15,41 & 15,50 & 10,12 \\
\hline Titoli con scadenza tra 1 e 5 anni & 1,40 & 1,35 & 1,35 & 1,51 & 1,85 & 2,84 & 4,79 & 7,04 & 6,48 & 5,35 & 3,40 \\
\hline Titoli con scadenza superiore ai 5 anni & 0,86 & 0,64 & 0,50 & 0,73 & 1,40 & 1,54 & 2,84 & 3,26 & 3,45 & 4,98 & 2,02 \\
\hline Prestiti allocati nella fascia a vista & 50,11 & 53,58 & 49,76 & 37,87 & 34,08 & 33,70 & 33,18 & 31,40 & 29,60 & 29,50 & 38,28 \\
\hline Prestiti con scadenza inferiore a 1 anno & 25,52 & 27,46 & 27,33 & 35,95 & 41,67 & 41,70 & 38,20 & 33,55 & 33,48 & 32,65 & 33,75 \\
\hline Prestiti con scadenza tra 1 e 5 anni & 8,71 & 5,20 & 5,77 & 7,43 & 6,67 & 6,73 & 6,16 & 6,99 & 6,92 & 7,24 & 6,78 \\
\hline Prestiti con scadenza superiore ai 5 anni & 7,95 & 5,00 & 7,06 & 7,85 & 5,18 & 5,46 & 4,22 & 4,40 & 4,67 & 4,78 & 5,66 \\
\hline Passività per cassa & 2006 & 2007 & 2008 & 2009 & 2010 & 2011 & 2012 & 2013 & 2014 & 2015 & media \\
\hline Conti correnti passivi da clientela & 44,36 & 42,98 & 44,22 & 48,54 & 47,08 & 43,89 & 40,26 & 41,23 & 45,53 & 49,45 & 44,75 \\
\hline Altre componenti a vista & 8,27 & 10,21 & 8,29 & 8,16 & 9,85 & 10,33 & 12,14 & 7,76 & 7,43 & 7,68 & 9,01 \\
\hline Altra raccolta inferiore a 1 anno & 14,04 & 12,54 & 9,12 & 5,88 & 7,49 & 10,65 & 16,47 & 22,29 & 18,50 & 16,73 & 13,37 \\
\hline Altra raccolta tra 1 e 5 anni & 1,23 & 0,67 & 0,90 & 0,87 & 0,77 & 1,01 & 1,59 & 2,05 & 2,75 & 5,11 & 1,70 \\
\hline Altra raccolta superiore ai 5 anni & 0,42 & 0,19 & 0,32 & 0,45 & 0,45 & 0,42 & 0,39 & 0,47 & 0,61 & 0,78 & 0,45 \\
\hline Titoli di debito con scadenza inferiore a 1 anno & 24,15 & 25,89 & 29,19 & 27,42 & 21,79 & 18,96 & 15,42 & 13,63 & 12,11 & 9,67 & 19,82 \\
\hline Titoli di debito con scadenza tra 1 e 5 anni & 6,84 & 6,88 & 7,20 & 7,76 & 11,32 & 13,77 & 12,93 & 11,85 & 12,14 & 9,56 & 10,03 \\
\hline Titoli di debito con scadenza superiore ai 5 anni & 0,71 & 0,63 & 0,75 & 0,91 & 1,24 & 0,97 & 0,79 & 0,73 & 0,95 & 1,02 & 0,87 \\
\hline
\end{tabular}

Fonte: Elaborazioni dell'Autore su dati di bilancio al 31/12 di ogni anno.

Le caratteristiche e la dinamica nel tempo della struttura per scadenza dei key-rates ha avuto un forte impatto sull'esposizione al rischio di tasso di interesse del portafoglio bancario. La tabella 11 riporta i livelli al 31/12 dei vari anni oggetto di analisi.

Le evidenze empiriche mostrano che la struttura per scadenza dei key-rates ha subito una tendenziale di riduzione dei livelli in corrispondenza di tutti i nodi ed in particolare su quelli a breve e medio termine che ha condotto ad una maggiore dell'inclinazione della stessa. A partire dal 31/12/2009 si osservano in corrispondenza di specifiche fasce temporali livelli dei key-rates inferiori ai 200 punti base che comportano l'applicazione del vincolo di non negatività per lo scenario parallelo dei 200 punti base. A partire dallo stesso anno il vincolo di non negatività è stato applicato anche al metodo dei percentili come si evince dal confronto della Tabella 11 con le successive Tabelle 12 e 13. Nello specifico la Tabella 12 riporta per ogni anno oggetto di analisi gli scenari associati al $99^{\circ}$ e al $1^{\circ}$ percentile delle variazioni su base annue dei tassi di interesse relative ai 5 anni precedenti la data di valutazione. Lo scenario del $1^{\circ}$ percentile non è corretto per tener conto del vincolo di non negatività. La Tabella 13 riporta, invece, lo scenario parallelo dei -200 punti base e quello associato al $1^{\circ}$ percentile corretto per il vincolo di non negatività.

Inoltre, negli ultimi due anni si registrano valori negativi dei key-rates. In particolare al 31/12/2014 solo sulla fascia temporale "Fino ad 1 mese" mentre al 31/12/2015 nelle fasce temporali che coprono l'orizzonte temporale dei 6 mesi e nella fascia temporale "Da 1 anno a 2 anni". Il tale caso ai fini dell'applicazione del vincolo di non negatività si fa riferimento a quanto riportato nella precedente nota 5. Lo scenario dei -200 punti base e quello del $1^{\circ}$ percentile sono, quindi, caratterizzati da variazioni positive di pari ampiezza al livello dello stesso key-rates vigente alla data di valutazione in modo tale da condurre la struttura per scadenza degli stessi key-rates ad assumere valori non negativi. È interessante osservare, infine, che negli ultimi due anni oggetto di analisi lo scenario dei -200 punti base e quello del $1^{\circ}$ percentile presentano variazioni di pari ampiezza su tutti i nodi della struttura per scadenza dei key-rates dato il basso livello dei tassi di interesse e la conseguente applicazione del vincolo di non negatività su tutte le fasce temporali della matrice per scadenza e per data di riprezzamento. 
Tabella 11. struttura per scadenza dei key-rates associati alle varie fasce temporali della matrice per scadenza e per data di riprezzamento al 31/12 di ogni anno.

(valori percentuali)

\begin{tabular}{|c|c|c|c|c|c|c|c|c|c|c|c|c|c|c|}
\hline \multirow{2}{*}{$\begin{array}{c}\text { Data di } \\
\text { valutazione }\end{array}$} & \multicolumn{14}{|c|}{ Fasce temporali } \\
\hline & A vista & $\begin{array}{c}\text { fino a } \\
1 \mathrm{~m}\end{array}$ & $1 m-3 m$ & $3 m-6 m$ & $6 m-1 a$ & $1 a-2 a$ & $2 a-3 a$ & $3 a-4 a$ & $4 a-5 a$ & $5 a-7 a$ & $7 a-10 a$ & $10 a-15 a$ & $15 a-20 a$ & $\begin{array}{c}\text { Oltre } \\
20 \mathrm{a}\end{array}$ \\
\hline $31 / 12 / 2006$ & 3,69 & 3,62 & 3,66 & 3,79 & 3,95 & 4,10 & 4,13 & 4,13 & 4,13 & 4,14 & 4,17 & 4,24 & 4,29 & 4,31 \\
\hline $31 / 12 / 2007$ & 3,92 & 4,18 & 4,49 & 4,70 & 4,73 & 4,63 & 4,54 & 4,53 & 4,54 & 4,58 & 4,67 & 4,80 & 4,89 & 4,91 \\
\hline $31 / 12 / 2008$ & 2,35 & 2,45 & 2,79 & 2,93 & 3,02 & 2,72 & 2,86 & 3,04 & 3,18 & 3,36 & 3,61 & 3,85 & 3,88 & 3,76 \\
\hline $31 / 12 / 2009$ & 0,41 & 0,40 & 0,56 & 0,84 & 1,13 & 1,59 & 2,06 & 2,41 & 2,68 & 2,99 & 3,43 & 3,82 & 4,02 & 4,05 \\
\hline $31 / 12 / 2010$ & 0,82 & 0,65 & 0,89 & 1,10 & 1,37 & 1,45 & 1,75 & 2,08 & 2,34 & 2,70 & 3,12 & 3,50 & 3,67 & 3,68 \\
\hline $31 / 12 / 2011$ & 0,63 & 0,76 & 1,18 & 1,48 & 1,79 & 1,37 & 1,35 & 1,45 & 1,63 & 1,91 & 2,24 & 2,57 & 2,68 & 2,65 \\
\hline $31 / 12 / 2012$ & 0,13 & 0,09 & 0,15 & 0,25 & 0,43 & 0,35 & 0,42 & 0,54 & 0,69 & 0,95 & 1,37 & 1,83 & 2,09 & 2,20 \\
\hline $31 / 12 / 2013$ & 0,45 & 0,20 & 0,26 & 0,34 & 0,48 & 0,48 & 0,66 & 0,89 & 1,13 & 1,49 & 1,95 & 2,41 & 2,65 & 2,73 \\
\hline $31 / 12 / 2014$ & 0,14 & $-0,01$ & 0,04 & 0,12 & 0,25 & 0,17 & 0,20 & 0,26 & 0,32 & 0,45 & 0,68 & 1,01 & 1,24 & 1,37 \\
\hline $31 / 12 / 2015$ & $-0,13$ & $-0,24$ & $-0,17$ & $-0,09$ & 0,00 & $-0,04$ & 0,01 & 0,12 & 0,26 & 0,47 & 0,82 & 1,23 & 1,48 & 1,59 \\
\hline
\end{tabular}

Fonte: Elaborazioni dell'Autore su dati Datastream.

Tabella 12: scenari del $99^{\circ}$ e del $1^{\circ}$ percentile associati alle varie fasce temporali della matrice per scadenza e per data di riprezzamento al 31/12 di ogni anno

(valori percentuali)

\begin{tabular}{|c|c|c|c|c|c|c|c|c|c|c|c|c|c|c|c|}
\hline \multirow{2}{*}{$\begin{array}{c}\text { Data di } \\
\text { valutazione }\end{array}$} & \multirow[b]{2}{*}{ percentile } & \multicolumn{14}{|c|}{ Fasce temporali } \\
\hline & & $\begin{array}{c}\text { A } \\
\text { vista }\end{array}$ & $\begin{array}{c}\text { fino a } \\
1 \mathrm{~m}\end{array}$ & $\begin{array}{l}1 \mathrm{~m}- \\
3 \mathrm{~m}\end{array}$ & $\begin{array}{l}3 \mathrm{~m}- \\
6 \mathrm{~m}\end{array}$ & $\begin{array}{c}6 \mathrm{~m}- \\
1 \mathrm{a}\end{array}$ & $\begin{array}{l}1 \mathrm{a}- \\
2 \mathrm{a}\end{array}$ & $\begin{array}{l}2 \mathrm{a}- \\
3 \mathrm{a}\end{array}$ & $\begin{array}{l}3 a- \\
4 a\end{array}$ & $\begin{array}{c}4 a- \\
5 a\end{array}$ & $\begin{array}{c}5 a- \\
7 a\end{array}$ & $\begin{array}{l}7 \mathrm{a}- \\
10 \mathrm{a}\end{array}$ & $\begin{array}{l}10 \mathrm{a}- \\
15 \mathrm{a}\end{array}$ & $\begin{array}{l}15 a- \\
20 a\end{array}$ & $\begin{array}{l}\text { Oltre } \\
20 \mathrm{a}\end{array}$ \\
\hline \multirow[t]{2}{*}{$31 / 12 / 2006$} & $99^{\circ}$ & 1,28 & 1,25 & 1,26 & 1,33 & 1,44 & 1,52 & 1,48 & 1,42 & 1,35 & 1,24 & 1,07 & 0,92 & 0,82 & 0,77 \\
\hline & $1^{\circ}$ & $-2,03$ & $-1,53$ & $-1,46$ & $-1,42$ & $-1,69$ & $-2,04$ & $-2,18$ & $-2,11$ & $-2,01$ & $-1,87$ & $-1,64$ & $-1,39$ & $-1,25$ & $-1,20$ \\
\hline \multirow[t]{2}{*}{$31 / 12 / 2007$} & $99^{\circ}$ & 1,37 & 1,26 & 1,38 & 1,36 & 1,45 & 1,52 & 1,50 & 1,43 & 1,36 & 1,24 & 1,08 & 0,92 & 0,82 & 0,79 \\
\hline & $1^{\circ}$ & $-1,37$ & $-1,24$ & $-1,27$ & $-1,40$ & $-1,69$ & $-2,04$ & $-2,17$ & $-2,12$ & $-2,02$ & $-1,88$ & $-1,67$ & $-1,42$ & $-1,26$ & $-1,21$ \\
\hline \multirow[t]{2}{*}{$31 / 12 / 2008$} & $99^{\circ}$ & 1,41 & 1,27 & 1,39 & 1,37 & 1,45 & 1,53 & 1,51 & 1,43 & 1,35 & 1,23 & 1,06 & 0,90 & 0,83 & 0,79 \\
\hline & $1^{\circ}$ & $-1,55$ & $-1,63$ & $-1,69$ & $-1,59$ & $-1,45$ & $-1,63$ & $-1,43$ & $-1,25$ & $-1,17$ & $-1,18$ & $-1,17$ & $-1,19$ & $-1,21$ & $-1,24$ \\
\hline \multirow[t]{2}{*}{$31 / 12 / 2009$} & $99^{\circ}$ & 1,37 & 1,26 & 1,38 & 1,36 & 1,45 & 1,52 & 1,50 & 1,43 & 1,36 & 1,24 & 1,08 & 0,92 & 0,82 & 0,79 \\
\hline & $1^{\circ}$ & $-3,98$ & $-4,32$ & $-4,41$ & $-4,33$ & $-4,20$ & $-3,72$ & $-3,19$ & $-2,72$ & $-2,37$ & $-1,91$ & $-1,50$ & $-1,23$ & $-1,21$ & $-1,23$ \\
\hline \multirow[t]{2}{*}{$31 / 12 / 2010$} & $99^{\circ}$ & 1,37 & 1,26 & 1,38 & 1,36 & 1,45 & 1,52 & 1,50 & 1,43 & 1,36 & 1,24 & 1,08 & 0,92 & 0,82 & 0,79 \\
\hline & $1^{\circ}$ & $-3,98$ & $-4,32$ & $-4,41$ & $-4,33$ & $-4,20$ & $-3,72$ & $-3,19$ & $-2,72$ & $-2,37$ & $-1,91$ & $-1,50$ & $-1,20$ & $-1,07$ & $-1,15$ \\
\hline \multirow[t]{2}{*}{$31 / 12 / 2011$} & $99^{\circ}$ & 1,31 & 1,24 & 1,38 & 1,33 & 1,24 & 1,19 & 1,10 & 1,03 & 0,98 & 0,91 & 0,82 & 0,76 & 0,75 & 0,75 \\
\hline & $1^{\circ}$ & $-3,98$ & $-4,32$ & $-4,40$ & $-4,33$ & $-4,20$ & $-3,71$ & $-3,20$ & $-2,72$ & $-2,36$ & $-1,92$ & $-1,50$ & $-1,19$ & $-1,06$ & $-1,15$ \\
\hline \multirow[t]{2}{*}{$31 / 12 / 2012$} & $99^{\circ}$ & 0,97 & 0,88 & 0,83 & 0,81 & 0,86 & 0,92 & 0,95 & 0,89 & 0,80 & 0,70 & 0,53 & 0,47 & 0,46 & 0,47 \\
\hline & $1^{\circ}$ & $-3,98$ & $-4,32$ & $-4,41$ & $-4,33$ & $-4,20$ & $-3,72$ & $-3,19$ & $-2,72$ & $-2,37$ & $-1,91$ & $-1,59$ & $-1,53$ & $-1,57$ & $-1,61$ \\
\hline \multirow[t]{2}{*}{$31 / 12 / 2013$} & $99^{\circ}$ & 0,96 & 0,88 & 0,83 & 0,76 & 0,81 & 0,92 & 0,95 & 0,89 & 0,80 & 0,70 & 0,54 & 0,51 & 0,52 & 0,53 \\
\hline & $1^{\circ}$ & $-3,98$ & $-4,32$ & $-4,41$ & $-4,33$ & $-4,20$ & $-3,72$ & $-3,19$ & $-2,72$ & $-2,37$ & $-1,91$ & $-1,59$ & $-1,53$ & $-1,57$ & $-1,61$ \\
\hline \multirow[t]{2}{*}{$31 / 12 / 2014$} & $99^{\circ}$ & 0,96 & 0,88 & 0,83 & 0,76 & 0,81 & 0,92 & 0,95 & 0,89 & 0,80 & 0,69 & 0,54 & 0,51 & 0,52 & 0,53 \\
\hline & $1^{\circ}$ & $-1,77$ & $-1,57$ & $-1,73$ & $-1,62$ & $-1,45$ & $-1,28$ & $-1,48$ & $-1,60$ & $-1,63$ & $-1,61$ & $-1,54$ & $-1,53$ & $-1,57$ & $-1,61$ \\
\hline \multirow[t]{2}{*}{$31 / 12 / 2015$} & $99^{\circ}$ & 0,96 & 0,88 & 0,83 & 0,76 & 0,81 & 0,92 & 0,95 & 0,89 & 0,80 & 0,69 & 0,54 & 0,51 & 0,52 & 0,53 \\
\hline & $1^{\circ}$ & $-1,09$ & $-1,21$ & $-1,28$ & $-1,37$ & $-1,42$ & $-1,28$ & $-1,48$ & $-1,60$ & $-1,63$ & $-1,61$ & $-1,54$ & $-1,53$ & $-1,63$ & $-1,67$ \\
\hline
\end{tabular}

Fonte: Elaborazioni dell'Autore su dati Datastream. 


\begin{tabular}{|c|c|c|c|c|c|c|c|c|c|c|c|c|c|c|c|}
\hline \multirow{2}{*}{$\begin{array}{c}\text { Data di } \\
\text { valutazione }\end{array}$} & \multirow[b]{2}{*}{ scenario } & \multicolumn{14}{|c|}{ Fasce temporali } \\
\hline & & $\begin{array}{c}\text { A } \\
\text { vista }\end{array}$ & $\begin{array}{c}\text { fino a } \\
1 \mathrm{~m}\end{array}$ & $\begin{array}{l}1 \mathrm{~m}- \\
3 \mathrm{~m}\end{array}$ & $\begin{array}{c}3 \mathrm{~m}- \\
6 \mathrm{~m}\end{array}$ & $\begin{array}{c}6 \mathrm{~m}- \\
1 \mathrm{a}\end{array}$ & $\begin{array}{l}1 \mathrm{a}- \\
2 \mathrm{a}\end{array}$ & $\begin{array}{c}2 a- \\
3 a\end{array}$ & $\begin{array}{l}3 \mathrm{a}- \\
4 \mathrm{a}\end{array}$ & $\begin{array}{c}4 a- \\
5 a\end{array}$ & $\begin{array}{l}5 \mathrm{a}- \\
7 \mathrm{a}\end{array}$ & $\begin{array}{l}7 \mathrm{a}- \\
10 \mathrm{a}\end{array}$ & $\begin{array}{c}10 \mathrm{a}- \\
15 \mathrm{a}\end{array}$ & $\begin{array}{l}15 \mathrm{a}- \\
20 \mathrm{a}\end{array}$ & $\begin{array}{c}\text { Oltre } \\
20 \mathrm{a}\end{array}$ \\
\hline \multirow[t]{2}{*}{$31 / 12 / 2006$} & -200 & $-2,00$ & $-2,00$ & $-2,00$ & $-2,00$ & $-2,00$ & $-2,00$ & $-2,00$ & $-2,00$ & $-2,00$ & $-2,00$ & $-2,00$ & $-2,00$ & $-2,00$ & $-2,00$ \\
\hline & $1^{\circ}$ & $-2,03$ & $-1,53$ & $-1,46$ & $-1,42$ & $-1,69$ & $-2,04$ & $-2,18$ & $-2,11$ & $-2,01$ & $-1,87$ & $-1,64$ & $-1,39$ & $-1,25$ & $-1,20$ \\
\hline \multirow[t]{2}{*}{$31 / 12 / 2007$} & -200 & $-2,00$ & $-2,00$ & $-2,00$ & $-2,00$ & $-2,00$ & $-2,00$ & $-2,00$ & $-2,00$ & $-2,00$ & $-2,00$ & $-2,00$ & $-2,00$ & $-2,00$ & $-2,00$ \\
\hline & $1^{\circ}$ & $-1,37$ & $-1,24$ & $-1,27$ & $-1,40$ & $-1,69$ & $-2,04$ & $-2,17$ & $-2,12$ & $-2,02$ & $-1,88$ & $-1,67$ & $-1,42$ & $-1,26$ & $-1,21$ \\
\hline \multirow[t]{2}{*}{$31 / 12 / 2008$} & -200 & $-2,00$ & $-2,00$ & $-2,00$ & $-2,00$ & $-2,00$ & $-2,00$ & $-2,00$ & $-2,00$ & $-2,00$ & $-2,00$ & $-2,00$ & $-2,00$ & $-2,00$ & $-2,00$ \\
\hline & $1^{\circ}$ & $-1,55$ & $-1,63$ & $-1,69$ & $-1,59$ & $-1,45$ & $-1,63$ & $-1,43$ & $-1,25$ & $-1,17$ & $-1,18$ & $-1,17$ & $-1,19$ & $-1,21$ & $-1,24$ \\
\hline \multirow[t]{2}{*}{$31 / 12 / 2009$} & -200 & $-0,41$ & $-0,40$ & $-0,56$ & $-0,84$ & $-1,13$ & $-1,59$ & $-2,00$ & $-2,00$ & $-2,00$ & $-2,00$ & $-2,00$ & $-2,00$ & $-2,00$ & $-2,00$ \\
\hline & $1^{\circ}$ & $-0,41$ & $-0,40$ & $-0,56$ & $-0,84$ & $-1,13$ & $-1,59$ & $-2,06$ & $-2,41$ & $-2,37$ & $-1,91$ & $-1,50$ & $-1,23$ & $-1,21$ & $-1,23$ \\
\hline \multirow[t]{2}{*}{$31 / 12 / 2010$} & -200 & $-0,82$ & $-0,65$ & $-0,89$ & $-1,10$ & $-1,37$ & $-1,45$ & $-1,75$ & $-2,00$ & $-2,00$ & $-2,00$ & $-2,00$ & $-2,00$ & $-2,00$ & $-2,00$ \\
\hline & $1^{\circ}$ & $-0,82$ & $-0,65$ & $-0,89$ & $-1,10$ & $-1,37$ & $-1,45$ & $-1,75$ & $-2,08$ & $-2,34$ & $-1,91$ & $-1,50$ & $-1,20$ & $-1,07$ & $-1,15$ \\
\hline \multirow[t]{2}{*}{$31 / 12 / 2011$} & -200 & $-0,63$ & $-0,76$ & $-1,18$ & $-1,48$ & $-1,79$ & $-1,37$ & $-1,35$ & $-1,45$ & $-1,63$ & $-1,91$ & $-2,00$ & $-2,00$ & $-2,00$ & $-2,00$ \\
\hline & $1^{\circ}$ & $-0,63$ & $-0,76$ & $-1,18$ & $-1,48$ & $-1,79$ & $-1,37$ & $-1,35$ & $-1,45$ & $-1,63$ & $-1,91$ & $-1,50$ & $-1,19$ & $-1,06$ & $-1,15$ \\
\hline \multirow[t]{2}{*}{$31 / 12 / 2012$} & -200 & $-0,13$ & $-0,09$ & $-0,15$ & $-0,25$ & $-0,43$ & $-0,35$ & $-0,42$ & $-0,54$ & $-0,69$ & $-0,95$ & $-1,37$ & $-1,83$ & $-2,00$ & $-2,00$ \\
\hline & $1^{\circ}$ & $-0,13$ & $-0,09$ & $-0,15$ & $-0,25$ & $-0,43$ & $-0,35$ & $-0,42$ & $-0,54$ & $-0,69$ & $-0,95$ & $-1,37$ & $-1,53$ & $-1,57$ & $-1,61$ \\
\hline \multirow[t]{2}{*}{$31 / 12 / 2013$} & -200 & $-0,45$ & $-0,20$ & $-0,26$ & $-0,34$ & $-0,48$ & $-0,48$ & $-0,66$ & $-0,89$ & $-1,13$ & $-1,49$ & $-1,95$ & $-2,00$ & $-2,00$ & $-2,00$ \\
\hline & $1^{\circ}$ & $-0,45$ & $-0,20$ & $-0,26$ & $-0,34$ & $-0,48$ & $-0,48$ & $-0,66$ & $-0,89$ & $-1,13$ & $-1,49$ & $-1,59$ & $-1,53$ & $-1,57$ & $-1,61$ \\
\hline \multirow[t]{2}{*}{$31 / 12 / 2014$} & -200 & $-0,14$ & 0,01 & $-0,04$ & $-0,12$ & $-0,25$ & $-0,17$ & $-0,20$ & $-0,26$ & $-0,32$ & $-0,45$ & $-0,68$ & $-1,01$ & $-1,24$ & $-1,37$ \\
\hline & $1^{\circ}$ & $-0,14$ & 0,01 & $-0,04$ & $-0,12$ & $-0,25$ & $-0,17$ & $-0,20$ & $-0,26$ & $-0,32$ & $-0,45$ & $-0,68$ & $-1,01$ & $-1,24$ & $-1,37$ \\
\hline \multirow[t]{2}{*}{$31 / 12 / 2015$} & -200 & 0,13 & 0,24 & 0,17 & 0,09 & 0,00 & 0,04 & $-0,01$ & $-0,12$ & $-0,26$ & $-0,47$ & $-0,82$ & $-1,23$ & $-1,48$ & $-1,59$ \\
\hline & $1^{\circ}$ & 0,13 & 0,24 & 0,17 & 0,09 & 0,00 & 0,04 & $-0,01$ & $-0,12$ & $-0,26$ & $-0,47$ & $-0,82$ & $-1,23$ & $-1,48$ & $-1,59$ \\
\hline
\end{tabular}

Fonte: Elaborazioni dell'Autore su dati Datastream.

La seguente Tabella 14 riporta per i vari anni oggetto di analisi le evidenze empiriche concernenti la tipologia di esposizione al rischio in corrispondenza delle due metodologie di misurazione considerate rappresentate dallo scenario parallelo dei +/-200 punti base e dal metodo dei percentili. Nello specifico è riportato il numero di banche esposte a variazioni in aumento dei tassi di interesse, in diminuzione dei tassi di interesse e neutrali al rischio con l'indicazione del relativo valor medio dell'indicatore di rischio per le prime due tipologie di esposizione al rischio. L'analisi dei dati evidenzia un maggior numero di banche esposte a variazioni in diminuzione dei tassi di interesse. In termini di valori medi, relativi all'intero periodo oggetto di analisi, si registrano 20,10 e 22,00 banche esposte a variazioni in diminuzione dei tassi di interesse nel caso di adozione, rispettivamente, dello scenario parallelo e del metodo dei percentili. Le banche esposte ad un aumento dei tassi di interesse sono, invece, pari, rispettivamente, a 12,70 e 7,90. Per le banche neutrali al rischio si rilevano, infine, valori, rispettivamente, di 7,20 e 10,10. A livello di singoli anni si osserva, in corrispondenza di entrambe le metodologie di misurazione, un maggior numero di banche esposte a variazioni in diminuzione dei tassi di interesse dal 2006 al 2011. Successivamente dal 2012 al 2015 prevale, in generale, il numero di banche neutrali al rischio con l'eccezione, nel caso di adozione dello scenario parallelo dei +200 punti base, del 2013 e del 2014. La differente composizione in termini numerici registrata negli ultimi anni è, principalmente, riconducibile, da un lato, all'incremento della durata media delle attività, a seguito del maggior peso dei titoli a tasso fisso iscritti nel portafoglio bancario e, dall'altro lato, all'applicazione del vincolo di non negatività dato il basso livello dei tassi di interesse.

L'analisi dei dati evidenzia, inoltre, un livello dell'indicatore di rischio più alto per le banche esposte a variazioni in aumento dei tassi di interesse nel caso di adozione dello scenario parallelo dei +200 punti base rispetto al metodo dei percentili e viceversa per le banche esposte a variazioni in diminuzione dei tassi di interesse. In termini di valori medi ponderati sull'intero periodo le banche esposte a variazioni in aumento dei tassi di interesse presentano un indicatore di rischio pari al 12,24\% nel caso di adozione dello scenario parallelo dei +200 punti base e al 4,08\% nel caso, invece, di utilizzo del metodo dei percentili. Per le banche esposte a variazioni in diminuzione dei tassi di interesse si registrano, invece, valori medi ponderati per le due metodologie di misurazione considerate pari, rispettivamente, all' $8,43 \%$ e all' $8,86 \%$. A livello di singoli anni si osserva che le banche esposte a variazioni in aumento dei tassi di interesse sono caratterizzate, nell'intero periodo considerato, da un livello medio dell'indicatore di rischio più elevato nel caso di adozione dello scenario parallelo dei +200 punti base rispetto all'ipotesi di utilizzo del $99^{\circ}$ percentile. Nel caso di banche esposte, invece, a variazioni in diminuzione dei tassi di interesse si rileva, dal 2006 al 2013, con l'eccezione del 2008, un valore medio dell'indicatore di rischio più elevato nel caso di adozione del $1^{\circ}$ percentile rispetto allo scenario parallelo dei -200 punti base. Negli ultimi due anni oggetto di analisi, invece, i valori dell'indicatore di rischio per le banche esposte a variazioni in diminuzione dei tassi di interesse assumono lo stesso valore in corrispondenza delle due metodologie in questione. Ciò è riconducibile al fatto che gli scenari dei -200 punti base e del $1^{\circ}$ percentile sono caratterizzati da variazioni dei key-rates di uguale ampiezza data l'applicazione del vincolo di non negatività su tutte le fasce temporali della matrice per scadenza e per data di riprezzamento. È interessante osservare, infine, che si registra un maggior divario tra i livelli medi dell'indicatore di rischio calcolati mediante le due 
metodologie regolamentari nel caso di banche esposte a variazioni in aumento dei tassi di interesse rispetto al caso di banche esposte a variazioni in diminuzione dei tassi di interesse. Le ragioni alla base di tale evidenza sono riconducibili sostanzialmente a quanto riportato nel paragrafo 4 in relazione all'applicazione delle due metodologie regolamentari alla banca A e alla B.

Tabella 14. Tipologie di esposizione al rischio periodo 2006-2015

(livello dell'indicatore di rischio in valori percentuali) $(*)$

Scenario parallelo

Metodo dei percentili

\begin{tabular}{|c|c|c|c|c|c|}
\hline & & Numero banche & Livello medio indicatore & Numero banche & Livello medio indicatore \\
\hline \multirow{3}{*}{2006} & aumento & 16 & 13,45 & 15 & 5,28 \\
\hline & diminuzione & 24 & 11,23 & 25 & 11,85 \\
\hline & neutrale & 0 & & 0 & \\
\hline \multirow{3}{*}{2007} & aumento & 10 & 10,41 & 7 & 4,41 \\
\hline & diminuzione & 30 & 10,20 & 33 & 10,37 \\
\hline & neutrale & 0 & & 0 & \\
\hline \multirow{3}{*}{2008} & aumento & 9 & 13,94 & 6 & 5,20 \\
\hline & diminuzione & 31 & 8,70 & 31 & 5,99 \\
\hline & neutrale & 0 & & 3 & \\
\hline \multirow{3}{*}{2009} & aumento & 9 & 15,98 & 5 & 10,76 \\
\hline & diminuzione & 30 & 8,70 & 35 & 11,15 \\
\hline & neutrale & 1 & & 0 & \\
\hline \multirow{3}{*}{2010} & aumento & 6 & 8,65 & 2 & 7,52 \\
\hline & diminuzione & 34 & 9,92 & 38 & 11,08 \\
\hline & neutrale & 0 & & 0 & \\
\hline \multirow{3}{*}{2011} & aumento & 10 & 6,11 & 8 & 1,82 \\
\hline & diminuzione & 25 & 7,02 & 30 & 7,66 \\
\hline & neutrale & 5 & & 2 & \\
\hline \multirow{3}{*}{2012} & aumento & 12 & 10,11 & 8 & 2,38 \\
\hline & diminuzione & 9 & 2,72 & 9 & 2,99 \\
\hline & neutrale & 19 & & 23 & \\
\hline \multirow{3}{*}{2013} & aumento & 22 & 8,89 & 12 & 2,96 \\
\hline & diminuzione & 8 & 5,03 & 9 & 5,09 \\
\hline & neutrale & 10 & & 19 & \\
\hline \multirow{3}{*}{2014} & aumento & 16 & 10,14 & 9 & 3,20 \\
\hline & diminuzione & 8 & 1,34 & 8 & 1,34 \\
\hline & neutrale & 16 & & 23 & \\
\hline \multirow{3}{*}{2015} & aumento & 17 & 7,03 & 7 & 2,06 \\
\hline & diminuzione & 2 & 0,19 & 2 & 0,19 \\
\hline & neutrale & 21 & & 31 & \\
\hline \multirow{3}{*}{$\begin{array}{l}\text { Valori } \\
\text { medi }\end{array}$} & aumento & 12,70 & 10,24 & 7,90 & 4,08 \\
\hline & diminuzione & 20,10 & 8,43 & 22,00 & 8,86 \\
\hline & neutrale & 7,20 & & 10,10 & \\
\hline
\end{tabular}

Fonte: Elaborazioni dell'Autore su dati di bilancio al 31/12 di ogni anno e dati Datastream.

(*) Il valore dell'indicatore di rischio nei vari anni oggetto di analisi in corrispondenza di ciascuna metodologia è ottenuto come media semplice dei valori calcolati sulle singole banche. I valori medi sull'intero periodo sono ottenuti, invece, come medie ponderate nei vari anni per il numero di banche che presentano la specifica tipologia di esposizione al rischio nei vari anni.

La seguente Tabella 15 riporta per i vari anni oggetto di analisi le evidenze empiriche concernenti l'impatto del portafoglio titoli sull'esposizione complessiva al rischio di tasso di interesse del portafoglio bancario. Nel dettaglio, in corrispondenza delle due metodologie di misurazione considerate rappresentate dallo scenario parallelo dei +200 punti base e dal metodo dei percentili è riportato il numero di banche rientranti nelle ipotesi descritte nel precedente paragrafo 4 con l'indicazione del relativo valore medio dell'indicatore di rischio.

L'analisi dei dati evidenzia un maggior numero di banche rientranti nell'ipotesi 3 . In termini di valori medi relativi all'intero periodo oggetto di analisi le banche rientranti in tale ipotesi sono pari a 20,10 nel caso di adozione del metodo dello scenario parallelo e 22,00 nel caso, invece, di adozione del metodo dei percentili. È interessante osservare che l'ipotesi 3 coincide con le banche esposte a variazioni in diminuzione dei tassi di interesse di cui alla precedente Tabella 14. A livello di singoli anni si osserva, in corrispondenza di entrambe le metodologie di misurazione, un maggior numero di banche rientranti nell'ipotesi 3 dal 2006 al 2011. Successivamente, dal 2012 al 2015 prevale il numero di banche neutrali al rischio ad eccezione del 2013 e del 2014 dove nel caso di adozione dello scenario parallelo dei +200 punti base si registra un maggior numero di banche rientranti nell'ambito dell'ipotesi2. L'analisi delle banche esposte a variazioni in aumento dei tassi di interesse (ipotesi 1 e 2) evidenzia in termini di valori medi sull'intero periodo oggetto di analisi un maggior numero di banche rientranti nell'ipotesi 1 per lo scenario parallelo dei +200 punti base rispetto al metodo dei percentili $(7,00$ vs 5,70$)$ e nell'ipotesi 2 per il metodo dei percentili (3,90 vs 4,00). A livello di singoli anni dal 2006 al 2011 il numero di banche 
rientranti nell'ipotesi 1 è maggiore di quelle di cui alla ipotesi 2 ad eccezione del 2010, dove il numero di banche rientranti nelle due ipotesi è analogo per entrambe le metodologie di misurazione e del 2011, dove nel caso di adozione del metodo dei percentili il numero di banche rientranti nell'ipotesi 2 è maggiore. Dal 2012 al 2015 si osserva, invece, un maggior numero di banche rientranti nella ipotesi 2.

L'analisi dei dati evidenzia, inoltre, un livello dell'indicatore di rischio più alto per le banche rientranti nell'ipotesi 1 nel caso di adozione dello scenario parallelo dei +200 punti base e nell'ipotesi 3 nel caso di utilizzo, invece, del metodo dei percentili. In termini di valori medi ponderati sull'intero periodo nel caso di adozione dello scenario parallelo dei +200 punti base le banche rientranti nell'ipotesi 1 presentano un indicatore di rischio pari al 13,35\% rispetto alle ipotesi 2 e 3 per le quali l'indicatore di rischio si attesta, rispettivamente al 6,42\% e all' $8,43 \%$. Nel caso, invece di utilizzo del metodo dei percentili l'ipotesi3 presenta un valore medio ponderato dell'indicatore di rischio sull'intero periodo pari all' $8,86 \%$ rispetto al $6,05 \%$ e al 2,15\% osservato, rispettivamente, per le ipotesil e 2. A livello di singoli anni si osserva nel caso di adozione dello scenario parallelo dei +200 punti base un maggior livello dell'indicatore di rischio per le banche rientranti nell'ipotesi 1 ad eccezione del 2011. Nel caso, invece, di adozione del metodo dei percentili l'ipotesi 3 è caratterizzata da un livello più alto dell'indicatore di rischio negli anni dal 2006 a 2008 e dal 2011 al 2013.

Si segnala, inoltre, il forte peso del portafoglio titoli sul livello dell'indicatore di rischio nell'ambito dell'ipotesi2 in caso di adozione dello scenario parallelo dei +200 punti base. Il valore medio dell'indicatore di rischio sull'intero periodo relativo al portafoglio titoli è pari al $14,15 \%$ (a fronte di un incremento dei fondi propri del $-7,74 \%$ determinato dalla banca commerciale). Nel caso di utilizzo, invece, del metodo dei percentili l'indicatore di rischio calcolato sul portafoglio titoli si attesta su un livello più basso e pari al 5,23\% (a fronte di un incremento del -3,07\% della banca commerciale). L'analisi della dinamica nei vari anni oggetto di analisi nel caso di utilizzo dello scenario parallelo dei +200 punti base evidenzia livelli dell'indicatore di rischio relativi al solo portafoglio titoli abbastanza elevati dal 2009 al 2015 con valori che si collocano, ad eccezione del 2011, sempre al di sopra del 10\% e con un incremento nel 2014 e nel 2015, dove si osservano valori, rispettivamente, del 16,11\% e del $17,19 \%$. Nel caso, invece, di adozione del metodo dei percentili si rilevano o nell'ambito dell'ipotesi in questione valori decisamente più contenuti.

Limitato è, infine, l'impatto del portafoglio titoli nell'ambito dell'ipotesi 3 nella prospettiva di attenuazione dell'esposizione al rischio di tasso di interesse del portafoglio bancario determinata dalla banca commerciale. In tale ipotesi infatti, dato lo scenario di variazioni negative su base annua dei tassi di interesse a cui l'intera banca è esposta il portafoglio titoli determina, in generale, un incremento del valore economico dei fondi propri che attenua il decremento registrato sulla banca commerciale. In termini di valori medi calcolati sull'intero periodo oggetto di analisi si osserva nel caso di adozione dello scenario parallelo un valore dell'indicatore di rischio sull'intera banca del 8,43\% dato dalla somma del decremento sulla banca commerciale pari al $10,20 \%$ e l'incremento sul portafoglio titoli di proprietà pari al $-1,77 \%$. Nel caso, invece di adozione del metodo dei percentili l'indicatore di rischio sull'intera banca si attesta al 8,86\%, dato dalla somma del decremento sulla banca commerciale pari a 10,54\% e l'incremento sul portafoglio titoli pari al -1,68\%. L'analisi a livello dei vari anni conferma il basso impatto del portafoglio titoli in termini di incremento del valore economico dei fondi propri dato lo scenario di variazione in diminuzione dei tassi di interesse a cui è esposta la banca.

Tabella 16. Differenti ipotesi di impatto del portafoglio titoli sull'esposizione complessiva al rischio (livello dell'indicatore di rischio in valori percentuali) $(*)$

\begin{tabular}{|c|c|c|c|c|c|c|c|c|c|}
\hline \multirow[b]{2}{*}{ Anno } & \multirow[b]{2}{*}{ Ipotesi } & \multicolumn{4}{|c|}{ Scenario parallelo } & \multicolumn{4}{|c|}{ Metodo dei percentili } \\
\hline & & $\begin{array}{l}\text { Numero } \\
\text { banche }\end{array}$ & Intera banca & Titoli & $\begin{array}{c}\text { Banca } \\
\text { commerciale }\end{array}$ & $\begin{array}{l}\text { Numero } \\
\text { banche }\end{array}$ & $\begin{array}{l}\text { Intera } \\
\text { banca }\end{array}$ & Titoli & $\begin{array}{c}\text { Banca } \\
\text { commerciale }\end{array}$ \\
\hline \multirow{5}{*}{2006} & 1 & 16 & 13,45 & 3,02 & 10,44 & 11 & 6,70 & 1,53 & 5,17 \\
\hline & 2 & 0 & 0,00 & 0,00 & 0,00 & 4 & 1,37 & 2,27 & $-0,90$ \\
\hline & 3 & 24 & 11,23 & $-1,18$ & 12,41 & 25 & 11,85 & $-1,08$ & 12,92 \\
\hline & $4 a$ & 0 & 0,00 & 0,00 & 0,00 & 0 & 0,00 & 0,00 & 0,00 \\
\hline & $4 \mathrm{~b}$ & 0 & 0,00 & 0,00 & 0,00 & 0 & 0,00 & 0,00 & 0,00 \\
\hline \multirow{5}{*}{2007} & 1 & 8 & 12,79 & 3,37 & 9,43 & 5 & 6,05 & 2,72 & 3,33 \\
\hline & 2 & 2 & 0,89 & 7,07 & $-6,18$ & 2 & 0,31 & 1,48 & $-1,17$ \\
\hline & 3 & 30 & 10,20 & $-1,01$ & 11,20 & 33 & 10,37 & $-1,13$ & 11,50 \\
\hline & $4 a$ & 0 & 0,00 & 0,00 & 0,00 & 0 & 0,00 & 0,00 & 0,00 \\
\hline & $4 \mathrm{~b}$ & 0 & 0,00 & 0,00 & 0,00 & 0 & 0,00 & 0,00 & 0,00 \\
\hline \multirow{5}{*}{2008} & 1 & 8 & 15,27 & 2,54 & 12,73 & 6 & 5,20 & 1,96 & 3,25 \\
\hline & 2 & 1 & 3,34 & 3,55 & $-0,20$ & 0 & 0,00 & 0,00 & 0,00 \\
\hline & 3 & 31 & 8,70 & $-1,35$ & 10,04 & 31 & 5,99 & $-0,90$ & 6,88 \\
\hline & $4 a$ & 0 & 0,00 & 0,00 & 0,00 & 2 & $-1,51$ & $-0,31$ & $-1,20$ \\
\hline & $4 \mathrm{~b}$ & 0 & 0,00 & 0,00 & 0,00 & 1 & $-1,25$ & $-2,45$ & 1,20 \\
\hline \multirow{4}{*}{2009} & 1 & 7 & 18,25 & 3,56 & 14,69 & 3 & 16,40 & 2,43 & 13,98 \\
\hline & 2 & 2 & 8,05 & 11,13 & $-3,08$ & 2 & 2,29 & 4,52 & $-2,23$ \\
\hline & 3 & 30 & 8,70 & $-1,16$ & 9,86 & 35 & 11,15 & $-1,37$ & 12,51 \\
\hline & $4 a$ & 1 & $-0,15$ & $-0,10$ & $-0,05$ & 0 & 0,00 & 0,00 & 0,00 \\
\hline
\end{tabular}




\begin{tabular}{|c|c|c|c|c|c|c|c|c|c|}
\hline & $4 b$ & 0 & 0,00 & 0,00 & 0,00 & 0 & 0,00 & 0,00 & 0,00 \\
\hline \multirow{5}{*}{2010} & 1 & 3 & 10,80 & 5,63 & 5,17 & 1 & 11,27 & 6,23 & 5,04 \\
\hline & 2 & 3 & 6,50 & 11,39 & $-4,89$ & 1 & 3,78 & 11,16 & $-7,38$ \\
\hline & 3 & 34 & 9,92 & $-2,56$ & 12,48 & 38 & 11,08 & $-2,20$ & 13,28 \\
\hline & $4 a$ & 0 & 0,00 & 0,00 & 0,00 & 0 & 0,00 & 0,00 & 0,00 \\
\hline & $4 \mathrm{~b}$ & 0 & 0,00 & 0,00 & 0,00 & 0 & 0,00 & 0,00 & 0,00 \\
\hline \multirow{5}{*}{2011} & 1 & 7 & 6,90 & 2,76 & 4,14 & 3 & 2,89 & 0,58 & 2,31 \\
\hline & 2 & 3 & 4,28 & 8,58 & $-4,29$ & 5 & 1,17 & 3,40 & $-2,22$ \\
\hline & 3 & 25 & 7,02 & $-3,40$ & 10,42 & 30 & 7,66 & $-3,00$ & 10,66 \\
\hline & $4 a$ & 1 & $-1,97$ & $-1,49$ & $-0,48$ & 1 & $-0,54$ & 0,00 & $-0,54$ \\
\hline & $4 b$ & 4 & $-1,73$ & $-6,17$ & 4,44 & 1 & $-0,18$ & $-4,73$ & 4,55 \\
\hline \multirow{5}{*}{2012} & 1 & 4 & 11,99 & 3,85 & 8,15 & 3 & 1,98 & 0,88 & 1,10 \\
\hline & 2 & 8 & 9,17 & 12,78 & $-3,62$ & 5 & 2,62 & 5,73 & $-3,11$ \\
\hline & 3 & 9 & 2,72 & $-1,28$ & 4,00 & 9 & 2,99 & $-1,25$ & 4,24 \\
\hline & $4 a$ & 7 & $-3,91$ & $-1,71$ & $-2,20$ & 10 & $-4,99$ & $-2,04$ & $-2,95$ \\
\hline & $4 b$ & 12 & $-3,72$ & $-6,51$ & 2,79 & 13 & $-2,79$ & $-5,63$ & 2,84 \\
\hline \multirow{5}{*}{2013} & 1 & 6 & 19,30 & 9,76 & 9,54 & 4 & 4,90 & 2,64 & 2,26 \\
\hline & 2 & 16 & 4,98 & 14,83 & $-9,85$ & 8 & 1,99 & 6,33 & $-4,33$ \\
\hline & 3 & 8 & 5,03 & $-3,39$ & 8,42 & 9 & 5,09 & $-3,87$ & 8,96 \\
\hline & $4 a$ & 6 & $-3,28$ & $-1,28$ & $-1,99$ & 5 & $-4,00$ & $-2,69$ & $-1,32$ \\
\hline & $4 b$ & 4 & $-4,18$ & $-9,87$ & 5,69 & 14 & $-3,91$ & $-6,77$ & 2,86 \\
\hline \multirow{5}{*}{2014} & 1 & 6 & 11,50 & 4,82 & 6,68 & 2 & 2,16 & 0,61 & 1,55 \\
\hline & 2 & 10 & 9,33 & 16,11 & $-6,79$ & 7 & 3,49 & 6,50 & $-3,01$ \\
\hline & 3 & 8 & 1,34 & $-1,10$ & 2,44 & 8 & 1,34 & $-1,10$ & 2,44 \\
\hline & $4 a$ & 7 & $-2,86$ & $-1,13$ & $-1,73$ & 15 & $-3,63$ & $-1,73$ & $-1,90$ \\
\hline & $4 b$ & 9 & $-1,93$ & $-3,32$ & 1,39 & 8 & $-2,04$ & $-3,20$ & 1,16 \\
\hline \multirow{5}{*}{2015} & 1 & 5 & 10,74 & 6,26 & 4,48 & 1 & 1,79 & 0,00 & 1,79 \\
\hline & 2 & 12 & 5,48 & 17,19 & $-11,71$ & 6 & 2,11 & 5,84 & $-3,73$ \\
\hline & 3 & 2 & 0,19 & $-0,82$ & 1,01 & 2 & 0,19 & $-0,82$ & 1,01 \\
\hline & $4 a$ & 16 & $-5,34$ & $-3,40$ & $-1,94$ & 24 & $-7,05$ & $-3,52$ & $-3,53$ \\
\hline & $4 \mathrm{~b}$ & 5 & $-3,25$ & $-4,01$ & 0,76 & 7 & $-3,68$ & $-5,40$ & 1,72 \\
\hline \multirow{5}{*}{$\begin{array}{l}\text { Valori } \\
\text { medi }\end{array}$} & 1 & 7,00 & 13,35 & 4,15 & 9,20 & 3,90 & 6,05 & 1,84 & 4,21 \\
\hline & 2 & 5,70 & 6,42 & 14,15 & $-7,74$ & 4,00 & 2,16 & 5,23 & $-3,07$ \\
\hline & 3 & 20,10 & 8,43 & $-1,77$ & 10,20 & 22,00 & 8,86 & $-1,68$ & 10,54 \\
\hline & $4 a$ & 3,80 & $-4,07$ & $-2,20$ & $-1,87$ & 5,70 & $-5,21$ & $-2,54$ & $-2,67$ \\
\hline & $4 \mathrm{~b}$ & 3,40 & $-3,00$ & $-5,65$ & 2,65 & 4,40 & $-3,06$ & $-5,42$ & 2,36 \\
\hline
\end{tabular}

Fonte: Elaborazioni dell'Autore su dati di bilancio al 31/12 di ogni anno e dati Datastream.

(*) Il valore dell'indicatore di rischio nei vari anni oggetto di analisi in corrispondenza di ciascuna metodologia è ottenuto come media semplice dei valori calcolati sulle singole banche. I valori medi sull'intero periodo sono ottenuti, invece, come medie ponderate nei vari anni per il numero di banche che presentano la specifica tipologia di esposizione al rischio nei vari anni.

\section{Le determinanti dell'esposizione al rischio}

L'analisi effettuata evidenzia che, date le ipotesi sottostanti il framework di vigilanza prudenziale ${ }^{9}$, l'esposizione al rischio di tasso di interesse del portafoglio bancario è funzione di tre fattori principali:

i) la struttura per scadenza delle posizioni nette che dipende dalle strategie di $A L M$ poste in essere dalla banca;

ii) lo scenario di variazione su base annua dei key-rates utilizzato ai fini del calcolo dell'indicatore di rischio, che dipende dalla metodologia di misurazione adottata e dall'eventuale imposizione del vincolo di non negatività;

\footnotetext{
${ }^{9}$ Tali ipotesi, rappresentate dal numero e dall'ampiezza delle fasce temporali, dalla calibrazione dei coefficienti di duration e dai criteri di allocazione delle poste di bilancio nelle varie fasce temporali, sono state oggetto di analisi da parte della letteratura specialistica di riferimento indicata nel precedente paragrafo 2. La tematica, seppur di rilevante interesse in una prospettiva regolamentare, non ha, ad eccezione per banche che utilizzano metodologie più sofisticate, implicazioni in termini di stima del capitale interno da porre a presidio del rischio di tasso di interesse del portafoglio bancario. Ciò in quanto, in genere, le banche di piccola e media dimensione usano sottoporre, in sede ICAAP, all'Autorità di vigilanza nazionali una misura dell'esposizione al rischio in condizioni ordinarie e di stress calcolata mediante l'applicazione di differenti scenari di variazione dei key-rates su base annua che dipendono dalla metodologia di misurazione adottata fermo restando le suddette ipotesi sottostanti il framework regolamentare.
} 
iii) la dotazione patrimoniale di fondi propri della banca computabile al denominatore del rapporto, che, data la variazione di valore economico del portafoglio bancario, determina il livello dell'indicatore di rischio.

Inoltre, le evidenze empiriche sul sistema bancario italiano evidenziano che le banche, in genere, presentano nelle fasce temporali a medio termine (che vanno dalla fascia temporale "Da oltre 1 anno fino a 2 anni" a quella "Da 4 anni fino a 5 anni") posizioni nette negative dovute, principalmente, al peso dei depositi a vista che, come noto, sono classificati in un orizzonte temporale di 5 anni. In tali fasce temporali si collocano anche le altre forme tecniche di raccolta a tasso fisso emesse in scadenza nel medio termine. Le posizioni nette negative a seguito di un aumento (diminuzione) dei key-rates danno luogo a posizione nette ponderate negative (positive) e quindi ad un incremento (riduzione) del valore economico dei fondi propri. Nelle fasce temporali a lungo termine (che vanno dalla fascia temporale "Da oltre 5 a 7 anni" a quella "Oltre i 20 anni") si rilevano, invece, posizioni nette positive riconducibili, essenzialmente, alla scadenza delle quote capitale dei mutui a tasso fisso e dei titoli a tasso fisso iscritti nel portafoglio bancario in scadenza nel lungo termine. Le posizioni nette positive in caso di aumento (diminuzione) dei key-rates danno luogo a posizioni nette ponderate positive (negative) e quindi ad una riduzione (incremento) del valore economico dei fondi propri. Le posizioni nette positive e negative che si formano sulle fasce temporali a breve termine (che vanno dalla fascia temporale "Fino a 1 mese" a quella "Da 6 mesi a 1 anno") non impattano in maniera significativa sulla stima dell'indicatore di rischio dati i bassi coefficienti di duration ad esse associati. In queste fasce temporali rientrano le poste di bilancio a tasso variabile, quelle a tasso fisso in scadenza entro 1 anni e la parte dei depositi a vista distribuita in tale orizzonte temporale.

La struttura per scadenza delle posizioni nette impatta in modo differente sull'esposizione al rischio a seconda dello scenario di variazione su base annua dei key-rates utilizzato. Nel caso di adozione dello scenario parallelo dei +200 punti base si applicano variazioni della stessa ampiezza su tutte le fasce temporali. Il differente impatto di queste ultime sull'esposizione al rischio dipende, quindi, la peso della relativa posizione netta e dal coefficiente di duration ad esse associato. Nel caso, invece, di scenario parallelo di -200 punti base l'applicazione del vincolo di non negatività in periodi di bassi livelli dei tassi di interesse come quello attuale conduce all'applicazione di uno scenario di variazione su base annua caratterizzato da variazioni di differente ampiezza sulle varie fasce temporali, che, unitamente ai coefficienti di duration, possono amplificare/smorzare l'impatto delle posizioni nette sull'esposizione al rischio. Nel caso di adozione del metodo dei percentili sia lo scenario lo scenario associato al $1^{\circ}$ percentile sia quello associato al $99^{\circ}$ percentile sono caratterizzati, per costruzione, da differenti ampiezze sulle varie fasce temporali a prescindere dall'eventuale applicazione, per lo scenario del $1^{\circ}$ percentile del vincolo di non negatività.

Le strategie di $A L M$ poste in essere dalle banche determinano, quindi, la struttura per scadenza delle posizioni nette, sulla base dei criteri di classificazione definiti dalla normativa di vigilanza prudenziale che modificano la durata originaria delle poste di bilancio nella prospettiva del rischio di tasso di interesse del portafoglio bancario. Nel caso di una banca esposta a variazioni in aumento dei tassi di interesse una modifica, a parità di altre condizioni, della composizione delle attività verso una minore (maggiore) scadenza e/o un maggior (minor) peso di quelle a tasso variabile riduce (aumenta) la durata media dell'attivo, facendo, di conseguenza, diminuire (aumentare) il livello dell'indicatore di rischio. Viceversa, una modifica, a parità di altre condizioni, della composizione delle passività verso una minore (maggiore) scadenza e/o un maggior (minor) peso di quelle a tasso variabile riduce (aumenta) la durata media del passivo facendo, di conseguenza, aumentare (diminuire) il livello dell'indicatore di rischio. Nel caso, invece, di una banca esposta a variazioni in diminuzione dei tassi di interesse il livello dell'indicatore di rischio aumenta (diminuisce) a fronte di una differente composizione delle attività caratterizzata da una minore (maggiore) scadenza e/o da un maggior (minor) peso di quelle a tasso variabile e si riduce (aumenta) nel caso, invece, di una differente composizione delle passività caratterizzata da una minore (maggiore) scadenza e/o un maggior (minor) peso di quelle a tasso variabile.

La ristrutturazione, in termini di scadenza e data di revisione, delle componenti di bilancio tipiche di una banca commerciale non può essere, tuttavia, effettuato in tempi brevi. La stessa normativa di vigilanza prudenziale fissa, infatti, un orizzonte temporale di 1 anno ai fini della misurazione dell'esposizione al rischio. La variazione nella composizione in termini di scadenza e data di revisione dei titoli di proprietà iscritti nel portafoglio bancario avviene, invece, con una tempistica più breve rispetto a quelle delle componenti tradizionali del bilancio di una banca commerciale fermo restando le eventuali implicazioni a conto economico. Gli impatti dell'operatività in titoli dipendono, innanzitutto, dalla tipologia di titoli acquistati/venduti in termini di data di scadenza e di riprezzamento. L'acquisto di titoli a tasso fisso e a medio e lungo termine conduce, a parità di altre condizioni, ad un aumento della durata media dell'attivo. Tali titoli sono, infatti, iscritti nelle fasce temporali corrispondenti alla scadenza degli stessi. L'impatto sul livello dell'indicatore di rischio dipende dalla tipologia di esposizione al rischio. Nel caso di una banca esposta a variazioni in aumento dei tassi di interesse tale strategia determina un incremento dell'indicatore di rischio e, viceversa, nel caso di una banca esposta a variazioni in diminuzione dei tassi di interesse una diminuzione dell'indicatore di rischio fermo restando la tipologia di esposizione al rischio.

Ai fini di una valutazione dell'impatto complessivo occorre, tuttavia, analizzare la provenienza delle risorse finanziarie utilizzate per finanziare l'acquisto di tali componenti dell'attivo bancario. L'aumento dell'indicatore di rischio in caso di banca esposta a variazioni in aumento e la diminuzione nel caso, invece, di banca esposta a variazione in diminuzione dei tassi di interesse è maggiore se i titoli a tasso fisso a medio e lungo termine sono acquistati mediante risorse finanziarie ottenute da titoli in portafoglio in scadenza o da forme tecniche di raccolta a breve termine. Nel caso invece di utilizzo della raccolta a vista, distribuita come nota in un orizzonte di cinque anni, e/o di altre forme tecniche di raccolta a tasso fisso e a medio termine, l'impatto è più contenuto. L'utilizzo di risorse finanziarie fornite, invece, dagli azionisti impone di considerare nell'analisi il maggior livello di fondi propri al denominatore dell'indicatore di rischio.

Infine, la vendita di titoli a tasso fisso e a medio e lungo termine conduce, a parità di altre condizioni, ad una riduzione della durata media dell'attivo. Ciò determina nel caso di una banca esposta a variazioni in aumento dei tassi di 
interesse una riduzione dell'indicatore di rischio e, viceversa, nel caso di una banca esposta a variazioni in diminuzione dei tassi di interesse un incremento dell'indicatore di rischio fermo restando la tipologia di esposizione al rischio. Ai fini di una valutazione dell'impatto complessivo occorre, tuttavia, analizzare le modalità di utilizzo delle risorse finanziarie ottenute dalla vendita dei titoli. Se, ad esempio, le stesse sono detenute in cassa oppure reinvestite in operazioni a breve termine e/o a tasso variabile si ha, effettivamente, una riduzione dell'indicatore di rischio nel caso di banche esposte a variazioni in aumento dei tassi di interesse e viceversa un aumento nel caso di banche, invece, esposte a variazioni in diminuzione dei tassi di interesse. Nel caso contrario occorre, invece, analizzare le caratteristiche delle nuove operazioni di impiego in termini di data di scadenza e di revisione del tasso di interesse.

L'operatività in titoli potrebbe, infine, condurre al fenomeno dell'inversione della tipologia di esposizione al rischio. Si tratta, ad esempio, di banche esposte a variazioni in diminuzione dei tassi di interesse che a seguito dell'acquisto di titoli a tasso fisso e a medio e lungo termine diventano esposte a variazioni in aumento dei tassi di interesse oppure di banche esposte a variazioni in aumento dei tassi di interesse che a seguito della vendita di titoli a tasso fisso e lungo termine diventano esposte a variazioni in diminuzione dei tassi di interesse.

\section{L'utilizzo dei derivati di copertura nell'ambito delle strategie di $A L M$}

La variazione della composizione delle poste di bilancio tra tasso fisso e variabile al fine di contenere l'esposizione al rischio può, tuttavia, avvenire, in tempi più brevi, mediante il ricorso a specifici derivati di copertura. Le banche possono coprire i mutui a tasso fisso mediante amortizing interest rate swap trasformando, di fatto, il tasso fisso applicato a tali componenti dell'attivo bancario in un tasso variabile ${ }^{10}$. La modellizzazione del derivato nella matrice per scadenza e per riprezzamento annulla l'effetto delle quote capitali del mutuo a tasso fisso nelle varie fasce temporali riconducendo la somma delle stesse quote capitali, pari al debito residuo, in corrispondenza della prima data di revisione del tasso di interesse variabile.

Le banche, in genere, adottano le c.d. micro-coperture o macro-coperture di gruppo nelle quali l'oggetto coperto è costituito da un insieme di elementi omogenei sotto il profilo del rischio ad essi associato. I mutui vengono raggruppati in cluster con caratteristiche simili in termini di durata, piano di rimborso, tasso contrattuale e tipologia di prenditore Ad ogni cluster corrisponde un piano di rimborso aggregato su cui viene stipulato, in genere, un singolo amortizing interest rate swap. Si parla, in tal caso, di relazione 1 a $\mathrm{n}$ in quanto si ha 1 derivato di copertura ed $\mathrm{n}$ oggetti coperti. La rappresentazione del derivato nelle varie fasce temporali della matrice per scadenza e per data di riprezzamento avviene mediante l'iscrizione, da un lato, di una posizione lunga pari all'importo nozionale del derivato da segnalare in corrispondenza della fascia temporale relativa alla prima data di revisione del tasso di interesse e, dall'altro lato, di una serie di posizioni corte pari alle quote ammortizzate del derivato da segnalare in corrispondenza delle fasce temporali in cui avvengono i rimborsi dei vari mutui oggetto di copertura. La somma delle posizioni lunghe è pari a quella delle posizioni corte. L'iscrizione delle posizioni corte consente, invece, di compensare le quote capitali in scadenza dei mutui a tasso fisso oggetto di copertura annullando, di fatto, l'esposizione al rischio della banca sulle relative fasce temporali. La differenza tra micro coperture e macro coperture di gruppo sta nel fatto che nelle prime i mutui estinti o rinegoziati non possono essere sostituiti a differenza di quanto, invece, consentito per le macro-coperture di gruppo. Ciò ha evidenti implicazioni sul risultato di esercizio. L'impossibilità di sostituire il mutuo estinto o rinegoziato conduce ad un debito residuo dell'insieme dei mutui oggetto di copertura differente in termini di ammontare rispetto al nozionale del derivato ancora da ammortizzare. Ciò può minare l'efficacia della copertura ${ }^{11}$ con possibili implicazioni a conto economico a seconda della dinamica dei tassi di interesse registrata nel periodo di riferimento.

L'impatto sul livello dell'indicatore di rischio dipende dalla tipologia di esposizione al rischio: nel caso di una banca esposta a variazioni in aumento dei tassi di interesse si registra una riduzione del livello dell'indicatore di rischio e, viceversa, nel caso di una banca esposta a variazioni in diminuzione dei tassi di interesse si rileva un incremento del livello dell'indicatore di rischio fermo restando la tipologia di esposizione al rischio. La seguente Tabella 18 mostra il caso di una banca esposta a variazioni in aumento dei tassi di interesse con un indicatore di rischio pari nel caso di adozione dello scenario parallelo dei +200 punti base e del metodo dei percentili rispettivamente al 15,55\% e al 4,98\%. La copertura dei mutui a tasso fisso determina una modifica della struttura per scadenza delle posizioni nette e una conseguente riduzione dell'indicatore di rischio che si attesta, in corrispondenza delle due predette metodologie di misurazione, rispettivamente al $10,79 \%$ e al $3,08 \%$. In tale paragrafo si è fatto riferimento a posizione nette calcolate su banche del campione oggetto di analisi cosi come descritto nel paragrafo 4. I dati sui derivati di copertura sono, invece, delle esemplificazioni utilizzate a fini espositivi data l'impossibilità di reperire gli stessi con il dettaglio necessario dai dati di bilancio.

\footnotetext{
${ }^{10}$ La stipula di un contratto di amortizinginterest rate swap consente di scambiare periodicamente il differenziale dei flussi di interesse riferiti ad un dato capitale nozionale, che varia nel tempo secondo uno specifico piano di ammortamento. Nel caso specifico la banca trasferisce un flusso di interesse a tasso fisso ottenuto sui mutui oggetto di copertura alla controparte con cui ha stipulato il derivato e riceve dalla stessa un flusso di interesse a tasso varabile ancorato ad un parametro di riferimento.

${ }^{11}$ Le banche effettuano, in genere con una cadenza coincidente con la pubblicazione dei documenti di bilancio dei test di efficacia retrospettiva e prospettica. Il primo evidenzia il grado di efficacia della copertura raggiunto nel periodo oggetto di analisi. In altri termini esso misura di quanto i risultati effettivi si siano discostati dalla copertura perfetta. Il secondo misura, invece, l'efficacia attesa della copertura durante la vita residua della stessa. La mancata efficacia della copertura determina, in genere, la chiusura della stessa o l'unwinding del derivato.
} 


\begin{tabular}{|c|c|c|c|c|c|c|c|c|c|c|c|c|}
\hline \multicolumn{13}{|c|}{$\begin{array}{l}\text { Tabella 18. Banca esposta a variazioni in aumento dei tassi di interesse: impatto sull'indicatore di rischio della } \\
\text { copertura dei mutui a tasso fisso mediante amortizing interest rate swap } \\
(\mathrm{PN}, \Delta \mathrm{PN} \text { e } \Delta \mathrm{VE} \text { in migliaia di euro - key-rates } \mathrm{e} \text { IR in valori percentuali) }\end{array}$} \\
\hline $\begin{array}{c}\text { Fasce } \\
\text { temporali }\end{array}$ & $\begin{array}{l}\text { key- } \\
\text { rates }\end{array}$ & $\begin{array}{l}\text { Duration } \\
\text { (a) }\end{array}$ & $\begin{array}{l}\text { Posizione } \\
\text { netta (b) }\end{array}$ & $\begin{array}{l}2,00 \\
\text { (c) }\end{array}$ & $99^{\circ}(d)$ & $\begin{array}{c}\text { PNP } \\
(\mathbf{a})^{*}(\mathbf{b})^{*}(\mathbf{c})\end{array}$ & $\begin{array}{c}\text { PNP } \\
(\mathbf{a})^{*}(\mathbf{b})^{*}(\mathbf{d})\end{array}$ & $\begin{array}{l}\text { posizione } \\
\text { lunga (e) }\end{array}$ & $\begin{array}{l}\text { posizione } \\
\text { corta (f) }\end{array}$ & $\begin{array}{l}\text { PN } \\
(\mathbf{g})= \\
(\mathbf{a})+(\mathbf{e})- \\
(\mathbf{f})\end{array}$ & $\begin{array}{c}\text { PNP } \\
(\mathbf{g})^{*}(\mathbf{b})^{*}(\mathbf{c})\end{array}$ & $\begin{array}{c}\text { PNP } \\
(\mathbf{g})^{*}(\mathbf{b}) *(\mathbf{d})\end{array}$ \\
\hline A vista & 0,63 & 0,00 & 874.662 & 2,00 & 1,31 & 0 & 0 & & & 874.662 & 0 & 0 \\
\hline Fino a 1m & 0,76 & 0,04 & 478.580 & 2,00 & 1,24 & 383 & 237 & 53.187 & 1.011 & 530.756 & 425 & 263 \\
\hline $1 \mathrm{~m}-3 \mathrm{~m}$ & 1,18 & 0,16 & 957.160 & 2,00 & 1,38 & 3.063 & 2.113 & 104.210 & 2.023 & 1.059 .347 & 3.390 & 2.339 \\
\hline $3 m-6 m$ & 1,48 & 0,36 & -120.065 & 2,00 & 1,33 & -864 & -575 & & 3.070 & -123.135 & -887 & -590 \\
\hline $6 m-1 a$ & 1,79 & 0,71 & -135.335 & 2,00 & 1,24 & -1.922 & -1.191 & & 5.123 & -140.458 & -1.995 & -1.237 \\
\hline $1 a-2 a$ & 1,37 & 1,38 & -207.516 & 2,00 & 1,19 & -5.727 & -3.408 & & 9.879 & -217.395 & -6.000 & -3.570 \\
\hline $2 a-3 a$ & 1,35 & 2,25 & -207.516 & 2,00 & 1,10 & -9.338 & -5.136 & & 9.879 & -217.395 & -9.783 & -5.381 \\
\hline $3 a-4 a$ & 1,45 & 3,07 & -207.516 & 2,00 & 1,03 & -12.741 & -6.562 & & 9.879 & -217.395 & -13.348 & -6.874 \\
\hline $4 a-5 a$ & 1,63 & 3,85 & -207.516 & 2,00 & 0,98 & -15.979 & -7.830 & & 9.879 & -217.395 & -16.739 & -8.202 \\
\hline $5 \mathbf{a}-7 \mathbf{a}$ & 1,91 & 5,08 & 154.732 & 2,00 & 0,91 & 15.721 & 7.153 & & 17.668 & 137.064 & 13.926 & 6.336 \\
\hline $7 a-10 a$ & 2,24 & 6,63 & 232.097 & 2,00 & 0,82 & 30.776 & 12.618 & & 26.501 & 205.596 & 27.262 & 11.177 \\
\hline 10a-15a & 2,57 & 8,92 & 98.111 & 2,00 & 0,76 & 17.503 & 6.651 & & 20.829 & 77.282 & 13.787 & 5.239 \\
\hline $15 a-20 a$ & 2,68 & 11,21 & 98.111 & 2,00 & 0,75 & 21.996 & 8.249 & & 20.829 & 77.282 & 17.327 & 6.497 \\
\hline Oltre 20a & 2,65 & 13,01 & 98.111 & 2,00 & 0,75 & 25.528 & 9.573 & & 20.829 & 77.282 & 20.109 & 7.541 \\
\hline$\Delta \mathbf{V E}$ & & & & & & 68.398 & 21.893 & 157.397 & 157.397 & & 47.473 & 13.540 \\
\hline$I R=\Delta V E / F P$ & & & & & & $15,55 \%$ & $4,98 \%$ & & & & $10,79 \%$ & $3,08 \%$ \\
\hline
\end{tabular}

Fonte: Elaborazioni dell'Autore su dati di bilancio al 31/12/2011 e dati Datastream. I dati sui derivati sono esemplificazioni utilizzate a fini espositivi.

Le banche possono, inoltre, coprire i titoli obbligazionari a tasso fisso mediante la stipula di interest rate swap trasformando, di fatto, il tasso fisso applicato a tali componenti del passivo bancario in un tasso variabile ${ }^{12}$. La modellizzazione del derivato nella matrice per scadenza annulla l'effetto del valore contabile del titolo obbligazionario sulla fascia temporale associata alla relativa scadenza riconducendo lo stesso in corrispondenza della prima data di revisione del tasso di interesse variabile.

Le banche, in genere, adottano le c.d. micro-coperture specifiche, che associano ad ogni emissione obbligazionaria uno specifico contratto di interest rate swap. Si parla in tal caso di relazione 1 a 1 in quanto si ha 1 derivato di copertura ed 1 oggetto coperto. La rappresentazione dell'interest rate swap nelle varie fasce temporali della matrice per scadenza e per data di riprezzamento avviene mediante l'iscrizione da un lato, di una posizione lunga in corrispondenza della fascia temporale relativa alla data di scadenza dei titoli obbligazionari e, dall'altro lato, di una posizione corta in corrispondenza della fascia temporale relativa alla prima data di revisione del tasso di interesse. In tal modo è come se la banca avesse iscritto nel proprio bilancio mediante la posizione corta un'emissione obbligazionaria a tasso variabile di valore nozionale pari a quello del derivato. L'iscrizione della posizione lunga consente, invece, di compensare il valore contabile dei titoli obbligazionari a tasso fisso emessi annullando, di fatto, l'esposizione a medio termine della banca. L'impatto sul livello dell'indicatore di rischio dipende dalla tipologia di esposizione al rischio: nel caso di una banca esposta a variazioni in aumento dei tassi di interesse si registra un incremento del livello dell'indicatore di rischio, viceversa, nel caso di banca esposta a variazioni in diminuzione dei tassi di interesse si rileva una riduzione del livello dell'indicatore di rischio. La seguente Tabella 19 mostra il caso di una banca esposta a variazioni in diminuzione dei tassi di interesse con un indicatore di rischio pari nel caso di adozione dello scenario parallelo dei +200 punti base e del metodo dei percentili rispettivamente al 7,53\% e al 4,16\%. La copertura delle obbligazioni a tasso fisso determina una modifica della struttura per scadenza delle posizioni nette e una conseguente riduzione dell'indicatore di rischio che si attesta, in corrispondenza delle due predette metodologie di misurazione, rispettivamente al $4,16 \%$ e al $6,07 \%$.

${ }^{12}$ La stipula di un contratto di interest rate swap consente di scambiare periodicamente il differenziale dei flussi di interesse riferiti ad un dato capitale nozionale costante nel tempo. Nel caso specifico la banca paga un tasso variabile ancorato ad un dato parametro di riferimento alla controparte con cui ha stipulato il derivato da cui riceve il tasso fisso che paga ai sottoscrittori delle obbligazioni oggetto di copertura. 


\begin{tabular}{|c|c|c|c|c|c|c|c|c|c|c|c|c|}
\hline Fasce temporali & key-rates & $\begin{array}{l}\text { Duration } \\
\text { (a) }\end{array}$ & $\begin{array}{c}\text { Posizione } \\
\text { netta (b) }\end{array}$ & $-2,00$ (c) & $1^{\circ}(\mathrm{d})$ & $\underset{(\mathrm{PNP})}{\mathbf{P N}(\mathrm{b}) *(\mathbf{c})}$ & $\begin{array}{c}\text { PNP } \\
(\mathrm{a})^{*}(\mathrm{~b})^{*}(\mathrm{~d})\end{array}$ & $\begin{array}{l}\text { posizione } \\
\text { lunga (e) }\end{array}$ & $\begin{array}{l}\text { posizione } \\
\text { corta (f) }\end{array}$ & $\begin{array}{c}\text { PN } \\
(\mathbf{g})= \\
(\mathrm{a})+(\mathrm{e})-(\mathrm{f})\end{array}$ & $\underset{\left.(\mathrm{PNP})^{*(\mathrm{~b})}\right)^{*(\mathrm{c})}}{\mathbf{P N}}$ & $\begin{array}{c}\text { PNP } \\
(\mathrm{g})^{*}(\mathrm{~b})^{*}(\mathrm{~d})\end{array}$ \\
\hline A vista & 0,63 & 0 & -9.827 & $-0,63$ & $-0,63$ & 0 & 0 & & & -9.827 & 0 & 0 \\
\hline Fino a $1 \mathrm{~m}$ & 0,76 & 0,04 & 281.676 & $-0,76$ & $-0,76$ & -86 & -86 & & 100.000 & 181.676 & -55 & -55 \\
\hline $1 \mathrm{~m}-3 \mathrm{~m}$ & 1,18 & 0,16 & 563.352 & $-1,18$ & $-1,18$ & -1.064 & -1.064 & & & 563.352 & -1.064 & -1.064 \\
\hline $3 m-6 m$ & 1,48 & 0,36 & -105.755 & $-1,48$ & $-1,48$ & 563 & 563 & & & -105.755 & 563 & 563 \\
\hline $6 \mathrm{~m}-1 \mathrm{a}$ & 1,79 & 0,71 & -104.929 & $-1,79$ & $-1,79$ & 1.334 & 1.334 & & & -104.929 & 1.334 & 1.334 \\
\hline $1 a-2 a$ & 1,37 & 1,38 & -160.501 & $-1,37$ & $-1,37$ & 3.034 & 3.034 & & & -160.501 & 3.034 & 3.034 \\
\hline $2 a-3 a$ & 1,35 & 2,25 & -160.501 & $-1,35$ & $-1,35$ & 4.875 & 4.875 & & & -160.501 & 4.875 & 4.875 \\
\hline $3 a-4 a$ & 1,45 & 3,07 & -160.501 & $-1,45$ & $-1,45$ & 7.145 & 7.145 & & & -160.501 & 7.145 & 7.145 \\
\hline $4 a-5 a$ & 1,63 & 3,85 & -160.501 & $-1,63$ & $-1,63$ & 10.072 & 10.072 & 100.000 & & -60.501 & 3.797 & 3.797 \\
\hline $5 a-7 a$ & 1,91 & 5,08 & 20.893 & $-1,91$ & $-1,91$ & -2.027 & -2.027 & & & 20.893 & -2.027 & -2.027 \\
\hline $7 a-10 a$ & 2,24 & 6,63 & 31.340 & $-2,00$ & $-1,50$ & -4.156 & -3.117 & & & 31.340 & -4.156 & -3.117 \\
\hline $10 a-15 a$ & 2,57 & 8,92 & 8.661 & $-2,00$ & $-1,19$ & -1.545 & -919 & & & 8.661 & -1.545 & -919 \\
\hline $15 a-20 a$ & 2,68 & 11,21 & 8.661 & $-2,00$ & $-1,06$ & -1.942 & -1.029 & & & 8.661 & -1.942 & -1.029 \\
\hline Oltre 20a & 2,65 & 13,01 & 8.661 & $-2,00$ & $-1,15$ & -2.254 & -1.296 & & & 8.661 & -2.254 & -1.296 \\
\hline$\Delta \mathrm{VE}$ & & & & & & 13.951 & 17.486 & 100.000 & 100.000 & & 7.706 & 11.241 \\
\hline $\mathbf{I R}=\Delta \mathrm{VE} / \mathrm{FP}$ & & & & & & $7,53 \%$ & $9,43 \%$ & & & & $4,16 \%$ & $6,07 \%$ \\
\hline
\end{tabular}

Fonte: Elaborazioni dell'Autore su dati di bilancio al 31/12/2011 e dati Datastream. I dati sui derivati sono esemplificazioni utilizzate a fini espositivi.

Gli impatti delle strategie di copertura appena descritte sul livello dell'indicatore di rischio ipotizzano che la tipologia di esposizione al rischio rimanga invariata. Possono, tuttavia, registrarsi casi di inversione dell'esposizione al rischio: ad esempio banche esposte a variazioni in aumento dei tassi di interesse che a seguito della copertura dei mutui a tasso fisso diventano esposte a variazioni in diminuzione dei tassi di interesse o banche esposte a variazioni in diminuzione dei tassi di interesse che a seguito della copertura dei prestiti obbligazionari a tasso fisso diventano esposte a variazioni in aumento dei tassi di interesse.

\section{Conclusioni ed implicazioni di policy}

La corretta comprensione delle determinanti dell'esposizione al rischio di tasso di interesse del portafoglio bancario è di fondamentale importanza ai fini della definizione di una relativa policy di rischio basata sui concetti fondamentali del RAF. La normativa di vigilanza prudenziale prevede, infatti, che le banche debbano definire un quadro di riferimento per la determinazione della propensione al rischio, detto RAF, che fissi ex-ante gli obiettivi di rischio-rendimento che l'intermediario intende raggiungere e i conseguenti limiti operativi. Ai fini dell'implementazione del RAF il dettato regolamentare fornisce una serie di informazioni minimali sottolineando che l'effettiva articolazione dello stesso va, comunque, calibrata in base alle caratteristiche dimensionali e di complessità operativa di ciascuna banca. Il dettato normativo definisce i seguenti concetti rilevanti ai fini del RAF:

i) Risk Capacity (massimo rischio assumibile): rappresenta il livello massimo di rischio che una banca è tecnicamente in grado di assumere senza violare i requisiti regolamentari o gli altri vincoli imposti dagli azionisti o dall'autorità di vigilanza;

ii) Risk Appetite (obiettivo di rischio o propensione al rischio): è il livello di rischio (complessivo e per tipologia) che la banca intende assumere per il perseguimento dei suoi obiettivi strategici;

iii) Risk Tollerance (soglia di tolleranza): indica la devianza massima dal Risk Appetite consentita; la soglia di tolleranza è fissata in modo da assicurare in ogni caso alla banca margini sufficienti per operare, anche in condizioni di stress, entro il massimo rischio assumibile;

iv) Risk Profile (rischio effettivo): esprime il rischio effettivamente misurato in un determinato istante temporale;

v) Risk Limits (limiti di rischio): consiste nell'articolazione degli obiettivi di rischio in limiti operativi definiti, in linea con il principio di proporzionalità, per tipologia di rischio, unità e/o linee di business, linee di prodotto e tipologie di clienti.

L'approccio metodologico, delineato nel presente contributo, richiede alle banche di definire per ciascuna tipologia di rischio un insieme di indicatori su cui calibrare i suddetti concetti e, allo stesso tempo, formalizzare il legame tra gli stessi indicatori e l'operatività aziendale. Il limite di Risk Capacity devrebbe essere calibrato solo sugli indicatori per i quali è previsto un limite regolamentare e posto pari allo stesso limite regolamentare oppure con una versione più prudente dello 
stesso. Su eventuali break-down degli indicatori regolamentari rappresentativi delle singole linee di business oppure di particolari aspetti dell'attività aziendale definire, invece, solo l'obiettivo di Risk Appetite e il limite di Risk Tollerance. La banca può definire, eventualmente, anche una soglia di warning che segnala un eccessivo avvicinamento al limite di Risk Tollerance.

I vari indicatori regolamentari e gli eventuali break-down degli stessi devono essere tradotti in strumenti di indirizzo gestionali che consentano alle differenti unità di business di operare in linea con le strategie definite ex-ante dalla banca e alle strutture di controllo di monitorarne l'effettiva realizzazione ex-post. La banca deve, quindi, definire un sistema di limiti operativi che consenta di tenere costantemente monitorata l'esposizione al rischio al fine di attuare, in modo tempestivo, le eventuali misure correttive funzionali a riportare l'operatività bancaria entro valori coerenti con il percorso di avvicinamento all'obiettivo di Risk Appetite. In altre parole, occorre definire dei valori soglia, relativamente agli indicatori di rischio regolamentari e agli eventuali break-down degli stessi, il cui superamento comporta, a seconda della minore o maggiore gravità riscontrata l'attivazione di specifici processi aziendali. In definitiva, la stesura del RAF richiede la definizione di un processo, che dalla misurazione dell'attuale esposizione al rischio (Risk Profile) conduce, inizialmente, alla fissazione degli obiettivi di rischio (Risk Appetite) sulla base delle informazioni contenute nel budget e, successivamente, ne garantisce il raggiungimento mediante una appropriata policy di rischio, che fornisce gli strumenti metodologici ed operativi necessari, da un lato, ad orientare l'operatività delle varie linee di business verso il raggiungimento degli obiettivi strategici fissati ex-ante dalla banca, e dall'altro lato, a consentire alle strutture di controllo di svolgere una costante ed efficace attività di monitoraggio.

Nella prospettiva del rischio di tasso di interesse del portafoglio bancario la Risk Capacity andrebbe, quindi, calibrata sull'indicatore di rischio regolamentare calcolato sull'intera banca come rapporto tra la variazione di valore economico a seguito dello scenario di tasso ipotizzato e il livello dei fondi propri. In relazione, invece, alle due linee di business, rappresentate dalla banca commerciale e dal portafoglio titoli di proprietà si propone, invece, di fissare, solo l'obiettivo di Risk Appetite e il limite di Risk Tollerance in termini di capitale assorbito o di indicatore di rischio a seconda se si preferisce isolare o meno la dinamica dei fondi propri dall'attività operativa delle due linee di business. La dinamica dei fondi propri potrebbe, infatti, essere analizzata e monitorata ad un livello più alto di top management in una prospettiva di tipo più prettamente strategico lasciando alle due linee di business l'obbligo di confrontarsi esclusivamente con il capitale allocato alle stesse nell'ambito della loro specifica operatività.

Le scelte in termini di data di scadenza e di revisione del tasso di interesse dei nuovi impieghi erogati e delle nuove forme di raccolta emesse nonché dei titoli detenuti in portafoglio devono essere, quindi, calibrate al fine di perseguire gli obiettivi di rischio (Risk Appetite) fissati in sede di RAF, sulla base delle informazioni contenute nel budget, a partire dal livello attuale di rischio (Risk Profile). Il raggiungimento dell'obiettivo di Risk Appetite richiede, tuttavia, l'implementazione di strategie di ALM differenti a seconda: a) che l'esposizione al rischio iniziale dell'intera banca (Risk Profile) in termini di livelli dell'indicatore di rischio sia maggiore o minore dell'obiettivo di rischio (Risk Appetite); b) che l'intera banca sia esposta a variazioni in aumento o in diminuzione dei tassi di interesse o sia neutrale al rischio; c) del differente contributo delle due linee di business all'esposizione al rischio iniziale dell'intera banca (Risk Profile); e d) delle ipotesi fatte in relazione alla dinamica del livello dei fondi propri. Alcuni esempi possono essere utili a chiarire i concetti sopra esposti. La seguente Tabella 20 considera una banca che ha fissato sulla complessiva esposizione al rischio limiti di Risk Tollerance e di Risk Capacity pari, rispettivamente, al 16,00\% e il 20,00\% e l'obiettivo di Risk Appetite al 10,00\%. Sono riportati, inoltre, due differenti livelli di Risk Profile pari al 6,00\% e al 14,00\%, che sono, rispettivamente, più basso e più alto del suddetto obiettivo di Risk Appetite.

Tabella 20. Concetti alla base del Risk Appetite Framework

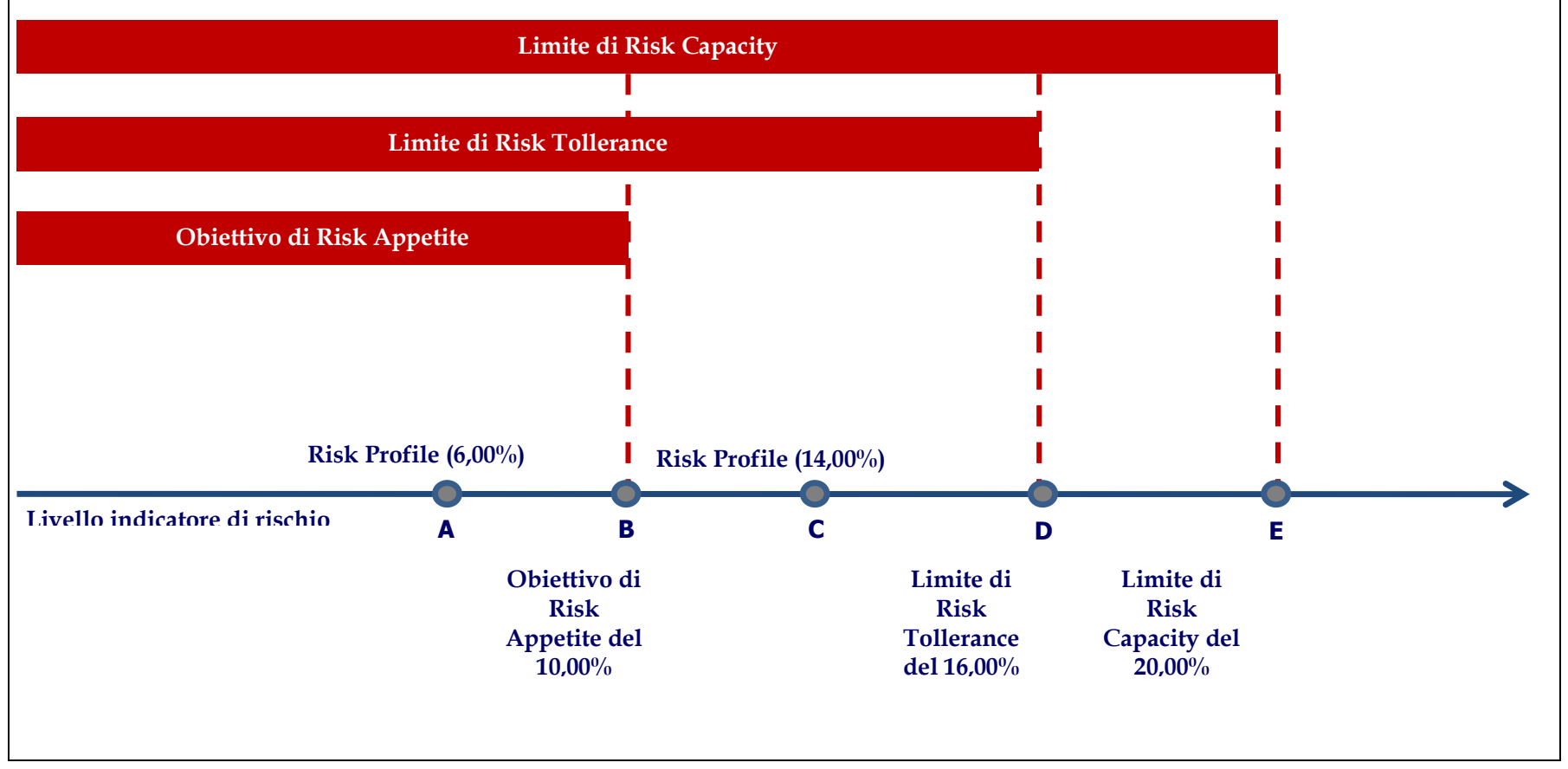


Nel caso di una intera banca esposta a variazioni in aumento dei tassi di interesse con livello di rischio (Risk Profile) pari al 14,00\% il raggiungimento dell'obiettivo di Risk Appetite, pari al 10\%, può essere ottenuto mediante una riduzione della durata media dell'attivo e/o un incremento di quella del passivo. L'interazione tra le due linee di business è, tuttavia, di particolare rilevanza ai fini dell'individuazione delle strategie di $A L M$ più appropriate. Le due linee di business, infatti, possono essere entrambe esposte a variazioni in aumento dei tassi di interesse (ipotesi 1 di cui al paragrafo 5) oppure compensarsi tra loro se la banca commerciale è esposta a variazioni in diminuzione dei tassi di interesse (ipotesi 2 di cui al paragrafo 5). Dal lato dell'attivo, nel caso in cui la banca commerciale è esposta a variazioni in aumento dei tassi di interesse con le due linee di business che, quindi, concorrono entrambe a determinare la tipologia di esposizione al rischio la definizione delle strategie di $A L M$ più appropriate deve considerare, innanzitutto, il differente contributo all'esposizione complessiva al rischio dell'aggregato dei mutui a tasso fisso e dei titoli a tasso fisso iscritti nel portafoglio bancario. L'implementazione di operazioni di copertura dei mutui a tasso fisso mediante la stipula di amortizing interest rate swap è una scelta strategica da tenere in considerazione per il raggiungimento dell'obiettivo di Risk Appetite soprattutto se la quota di tale forma tecnica di impiego è consistente ed il portafoglio titoli, invece, di dimensioni non elevate e/o caratterizzato, in prevalenza, da poste a tasso variabile. Viceversa in caso di una consistente quota di titoli a tasso fisso e a medio-lungo termine l'eventuale vendita degli stessi, fermo restando le possibili implicazioni a conto economico, può rappresentare la strategia più idonea al raggiungimento dell'obiettivo di Risk Appetite.

Nel caso in cui, invece, la banca commerciale è esposta a variazione in diminuzione dei tassi di interesse con le due linee di business che, quindi, si compensano tra loro la soluzione più adeguata per il raggiungimento dell'obiettivo di Risk Appetite è sicuramente quella di ridurre la componente a tasso fisso e medio lungo termine dei titoli detenuti in portafoglio, fermo restando le possibili implicazioni a conto economico derivanti da una eventuale vendita degli stessi, anziché coprire mediante l'utilizzo di amortizing interest rate swaps l'eventuale parte dei mutui a tasso fisso presenti nel portafoglio bancario. Gli stessi strumenti derivati utilizzati per le coperture delle obbligazioni a tasso fisso, rappresentati dagli interest rate swap, possono essere, invece, usati per attuare strategie di copertura sui titoli a tasso fisso iscritti nel portafoglio bancario sulla base delle stesse logiche descritte nel corso della trattazione.

I limiti di Risk Tollerance fissate sulle singole linee di business dovrebbero, comunque, guidare verso le strategie sopra delineate. Se tali limiti sono stati calibrati, infatti, in modo coerente con quello dell'indicatore di rischio regolamentare, fermo restando le ipotesi formulate sulla durata media del passivo e sulla dinamica dei fondi propri, nel caso di un eccessivo peso del portafoglio titoli di proprietà è presumibile che lo stesso assorba già un consistente ammontare di capitale collocandosi al ridosso del relativo limite di Risk Tollerance. Un'analoga considerazione può essere fatta per la banca commerciale nel caso in cui si rileva una quota significativa di mutui a tasso fisso. La banca potrebbe anche individuare appropriate strategie di $A L M$ volte a modificare la durata media del passivo rimodulandone la struttura per scadenza e per data di riprezzamento delle varie forme tecniche di raccolta ai fini del raggiungimento dell'obiettivo di Risk Appetite. Ciò può avvenire, ad esempio, ricorrendo a forme tecniche a tasso fisso e medio termine sia in sostituzione di quelle in scadenza sia per finanziarie nuovi impieghi a tasso variabile e/o a breve termine. Tale strategia è sicuramente adatta nel caso di banca commerciale esposta a variazioni in aumento dei tassi di interesse. Nel caso invece di banca commerciale esposta a variazioni in diminuzione dei tassi di interesse deve essere, invece, valutata con cautela onde evitare una eccessiva esposizione al rischio della singola linea di business in questione. Infine, un eventuale aumento della dotazione patrimoniale consentirebbe di ridurre l'esposizione al rischio senza modificare la struttura per scadenza e per data di riprezzamento delle poste di bilancio.

Viceversa, se il livello di Risk Profile è pari al 6,00\% il raggiungimento dell'obiettivo di Risk Appettite richiede l'implementazione di strategie di $A L M$ finalizzate ad aumentare la durata media dell'attivo e/o a ridurre quella del passivo. In tale circostanza, dal lato dell'attivo l'acquisto di titoli a tasso fisso e a lunga scadenza può essere considerata una strategia adeguata, fermo restando il rispetto di limite di Risk Tollerance associato alla stessa linea di business. L'acquisto di tali titoli deve essere, ovviamente, finanziamento mediante il ricorso a forme tecniche di raccolta a più breve scadenza e/o la vendita di titoli a breve termine e/o tasso variabile. L'eventuale erogazione di mutui a tasso fisso per allungare la durata media dell'attivo potrebbe, invece, essere ostacolata da una domanda di mercato orientata verso forme tecniche a tasso variabile. Dal lato del passivo, invece, la copertura delle obbligazioni a tasso fisso mediante interest rate swap oppure la sostituzione di parte della raccolta a vista, distribuita, come noto, nell'orizzonte temporale di 5 anni con depositi vincolati a breve termine consente di ridurre la durata media di tale aggregato del bilancio bancario e, allo stesso tempo, contribuire al raggiungimento dell'obiettivo di Risk Appetite. La riduzione della durata media del passivo è auspicabile soprattutto nel caso di una eccessiva esposizione a variazioni in diminuzione dei tassi di interesse della banca commerciale. Nel caso, invece, di una banca esposta a variazioni in diminuzione dei tassi di interesse (ipotesi 3 di cui al paragrafo 5) con un livello dell'indicatore di rischio Risk Profile pari a 14\% il raggiungimento dell'obiettivo di Risk Appetite pari al $10 \%$ può essere ottenuto mediante un aumento della durata media dell'attivo o una riduzione di quella del passivo. Viceversa, nel caso di un livello di Risk Profile pari al $6,00 \%$. Anche in tale caso l'analisi dell'esposizione al rischio delle due linee di business fornisce un utile supporto alla scelta delle più idonee strategie di $A L M$ da implementare.

L'analisi di come le due linee di business, rappresentate dal portafoglio titoli di proprietà e dalla banca commerciale, impattano sull'esposizione complessiva della banca è, di fondamentale importanza ai fini di una consapevole e prudente gestione del rischio. Al riguardo è interessante considerare il seguente caso esemplificativo di due banche in cui le due linee di business sono esposte a variazioni differenti dei tassi di interesse (in aumento per il portafoglio titoli e in diminuzione per la banca commerciale) compensandosi, quindi, tra loro ai fini del calcolo dell'indicatore di rischio regolamentare sull'intera banca. Consideriamo il caso in cui le due banche sono entrambe esposte a variazioni in aumento dei tassi di interesse (ipotesi 2 di cui al paragrafo 5) con un indicatore di rischio complessivo che si attesta per entrambe, ad esempio, al 3,5\%. Il breakdown dell'indicatore regolamentare per linee di business può, tuttavia, rilevare una differente virtuosità della struttura per 
scadenza delle due banche. Il livello dell'indicatore di rischio complessivo pari a 3,5\% potrebbe, infatti, essere per la prima banca la combinazione di una riduzione del valore economico sul portafoglio titoli del $22 \%$ compensata da un incremento del valore economico sulla banca commerciale, a seguito dell'applicazione dello scenario in aumento, del 17,5\%; per la seconda banca la combinazione, invece, di una riduzione di valore economico sul portafoglio titoli del $5 \%$ compensata da un incremento sulla banca commerciale del $2,5 \%$. In definitiva, l'indicatore di rischio regolamentare per l'intera banca potrebbe essere dato dalla combinazione di eccessive e/o contenute esposizioni sulle due linee di business con differenti implicazioni in termini di possibili strategie di ALM. Considerazioni del tutto analoghe possono essere effettuate nel caso in cui le due banche sono entrambe esposte a variazioni in diminuzione dei tassi di interesse (caso 3 di cui al paragrafo 5).

Nell'esempio sopra riportato la prima banca risulta caratterizzata da una eccessiva esposizione a variazioni in diminuzione dei tassi di interesse della banca commerciale che in uno scenario caratterizzato da livelli molto bassi della struttura per scadenza dei tassi di interesse come quello attuale è difficilmente riscontrabile nella realtà operativa. L'applicazione del vincolo di non negatività, in tali circostanze, smorza di fatto la riduzione di valore economico registrabile, in genere, sulle fasce temporali a medio termine determinando il fenomeno della neutralità al rischio che conduce ad un assorbimento di capitale interno pari zero. In tali circostanze la definizione delle strategie di $A L M$ deve tener conto, tuttavia, anche della possibile dinamica futura della struttura per scadenza dei tassi di interesse nel successivo orizzonte temporale di riferimento. Un eventuale spostamento verso l'alto della stessa potrebbe, infatti, non rendere più necessaria l'applicazione del vincolo di non negatività e rendere, allo stesso tempo, la banca esposta a variazioni in diminuzione dei tassi di interesse con la necessità di porre a presidio del rischio in questione il relativo capitale interno. Nell'ambito della definizione degli obiettivi di rischio in sede RAF va quindi data particolare attenzione non solo all'interazione tra le variabili micro riferite alla singola banca ma anche a quelle macro riconducibili, invece, alla dinamica delle variabili finanziarie e del sistema bancario nel suo complesso.

Infine, l'implementazione delle strategie di $A L M$ delineate nel corso della trattazione potrebbe condurre anche al fenomeno della c.d. inversione della tipologia di esposizione al rischio. Si tratta, nello specifico, di banche esposte a variazioni in aumento (diminuzione) dei tassi di interesse che a seguito dell'applicazione di strategie di ALM che comportano una riduzione (aumento) della durata media dell'attivo e/o un aumento (riduzione) di quella del passivo risultano esposte a variazioni in diminuzione (aumento) dei tassi di interesse. È in caso, ad esempio, di una banca esposta a variazioni in aumento (diminuzione) dei tassi di interesse che a seguito della vendita (acquisto) dei titoli a tasso fisso e/o copertura mediante appositi strumenti derivati dei mutui (obbligazioni) a tasso fisso risulta esposta a variazioni in diminuzione (aumento) dei tassi di interesse. Ai fini della definizione degli obiettivi di rischio in sede RAF date le ipotesi di budget va, quindi, tenuto in considerazione anche questo aspetto che potrebbe verificarsi a seguito di operazioni di una significativa consistenza e/o di un basso livello dell'esposizione al rischio iniziale (Risk Profile).

Il presupposto logico per l'applicazione di una policy del rischio di tasso di interesse del portafoglio bancario basata sui concetti fondamentali del RAF è, tuttavia, rappresentato da una adeguata diffusione della cultura del rischio tra tutte le funzioni aziendali interessate. Una maggiore consapevolezza delle implicazioni in termini di capitale interno da porre a presidio del rischio di tasso di interesse del portafoglio bancario derivante dagli impieghi erogati, dalle forme tecniche di raccolta emesse e dalla composizione del portafoglio titoli di proprietà contribuisce, infatti, a fornire alle strutture operative una visione più integrata e trasversale della banca facendo leva su una maggiore e più efficace interazione tra $i$ vari processi aziendali. Le tematiche riportate nel presente contributo sono di particolare rilevanza per gli operatori del settore, che devono non solo misurare l'esposizione al rischio della banca ma comprenderne, allo stesso tempo, le relative implicazioni gestionali e di business sulla base sia dell'attuale quadro normativo di riferimento sia delle eventuali proposte di modifica delineate dal Comitato di Basilea nel documento tecnico pubblicato nel recente mese di aprile. Inoltre, fornisce utili indicazioni ai regulators che devono monitorare l'esposizione al rischio del sistema bancario a livello sia micro che macro al fine, da un lato, di preservare la solidità delle singole banche e, dall'altro lato, di garantire la stabilità finanziaria globale e, allo stesso tempo, un'adeguata offerta di credito all'economia. Infine, rappresenta uno stimolo per la comunità accademica e, nello specifico, per gli studiosi di risk management collocati sulla linea di demarcazione tra ricerca teorica ed applicata ed incentivati a spostare più in avanti la frontiera dell'indagine scientifica sulle determinanti della relazione di interdipendenza tra l'attivo e il passivo bancario nella prospettiva di consentire una sempre più consapevole assunzione del rischio da parte degli intermediari.

\section{Igor Gianfrancesco}

\section{Bibliografia}

- Abdymomunov, A., Gerlach J., 2014. Stress testing interest rate risk exposure, in Journal of Banking and Finance 49, $287-301$.

- Banca d'Italia (2006), Nuove disposizioni di vigilanza prudenziale per le banche, Circolare n.263 del 27 dicembre 2006 e successivi aggiornamenti.

- Banca d'Italia (2008), Matrice dei Conti, Circolare 272 del 30 luglio 2008 e successivi aggiornamenti.

- Banca d'Italia (2013), Disposizioni di vigilanza prudenziale per le banche, Circolare n.285 del 17 dicembre 2013 e successivi aggiornamenti.

- $\quad$ Basel Committee on Banking Supervision (2004), Principles for Management and Supervision of Interest Rate Risk.

- Basel Committee on Banking Supervision (2006), International Convergence of Capital Measurement and Capital Standards: a Revised Framework. 
- Basel Committee on Banking Supervision (2009), Range of practices and issues in economic capital frameworks.

- $\quad$ Basel Committee on Banking Supervision (2016), Interest rate risk in the banking book.

- Committee of European Bankig Supervision (2006), Technical aspects of the management of interest rate risk arising from nontrading activities under the supervisory review process.

- Cocozza R, Curcio D., Gianfrancesco I. (2015a), Non-Maturity Deposits and banks' exposure to the interest rate risk: issues arising from the Basel regulatory frame work, in Journal of Risk, 17(5), 1-36.

- Cocozza R, Curcio D., Gianfrancesco I. (2015b), Measuring bank's interest rate risk: issues arising from regulatory and internal methodologies, Arcelli Centre for Monetary and Financial Studies, Working Paper series n.4, University LUISS Guido Carli Roma.

- Entrop, O., Memmel, C., Wilkens, M., and Zeisler, A. (2008), Analyzing the interest rate risk of banks using time series of accounting-based data: evidence from Germany, Deutsche Bundesbank Discussion Paper Series 2 no. 01.

- $\quad$ Entrop, O., Wilkens, M., and Zeisler, A. (2009), Quantifying the Interest Rate Risk of Banks: Assumptions Do Matter, European Financial Management 15(5), 1001-1018.

- Esposito L., Nobili A., Ropele T. (2013), The management of interest rate risk during the crisis: evidence from Italian banks, Working Paper della Banca d'Italia n. 933.

- European Banking Authority (2015), Guidelines on the management of interest rate risk arising from non-trading activities.

- Fiori, R., and Iannotti, S. (2007), Scenario Based Principal Component Value-at-Risk: An Application to Italian Bank's Interest Rate Risk Exposure, in Journal of Risk 9(3), 63-99.

- Houpt, J. V., and Embersit, J. A. (1991), A Method for Evaluating Interest Rate Risk in U.S. Commercial Banks, in Federal Reserve Bulletin, 77(2), 625-637.

- Sierra, G. E. (2009), Can an accounting-based duration model effectively measure interest rate sensitivity? paper disponibile sul sito SSRN al seguente link: http://papers.ssrn.com/sol3/papers.cfm? abstract_id=1488884.

- $\quad$ Sierra G. E., and Yeager T. J. (2004), What Does the Federal Reserve's Economic Value Model Tell Us About Interest Rate Risk at U.S. Community Banks? in The Federal Reserve Bank of St. Louis Review 86(6).

- Wright D. M., and Houpt J. V. (1996), An Analysis of Commercial Bank Exposure to Interest Rate Risk, in Federal Reserve Bulletin 82(2), 115-28. 


\title{
Implementazione della Fuzzy Logic per la gestione ottimale del portafoglio: la modellizzazione dell'avversione al rischio di un investitore attraverso tecniche di soft- computing
}

\author{
di Pier Giuseppe Giribone ${ }^{1}$, Ottavio Caligaris ${ }^{2}$, Simone Fioribello ${ }^{3}$ e Simone Ligato $^{1}$
}

\begin{abstract}
According to the classical theories for the optimal management of a financial assets portfolio, the decision-making process of evaluation of the convenience to invest in a product follows two sequential phases: the first one has a quantitative and objective nature, the second one has a qualitative and subjective nature. The former phase is characterized by the fact that it is independent from the investor's behavioral characteristics: in fact, this procedure quantifies, according to the Markowitz approach, the expected returns, volatilities, correlations in the assets being available on the market and covering a given investment horizon. These calculations generally lead to plot the efficient sets, within which the rational decision-maker chooses, depending on his risk aversion and any other characteristic factors, the optimal subjectively combinations. While the first phase, due to its quantitative nature, can be itself well modeled with traditional mathematical-statistical techniques, widely covered by the specific literature (such as efficient frontiers, multi-objective optimization, multiple regression analysis, CAPM, SML, alpha/beta analysis), the second one, being more subjective, requires a different approach. Always remaining within a quantitative framework, it is considered that the use of a soft-computing technique, such as Fuzzy Logic technique, can be considered a valid analysis tool.

The study is divided into two parts: the first section deals with the operating principles of the Fuzzy Logic, while in the second part a case study developed in Matlab has been shown, with reference to a prudent investor.
\end{abstract}

\section{Key Words:}

Fuzzy Logic (FL), Mamdani System, linguistic variables, soft-computing, investment decision

\section{Introduzione}

In accordo con le teorie classiche relative alla gestione ottimale di un portafoglio di asset finanziari, il processo decisionale di valutazione della convenienza nell'investire in un prodotto segue due fasi sequenziali: una di natura quantitativa-oggettiva ed una di natura qualitativa-soggettiva. La prima fase è indipendente dalle caratteristiche comportamentali dell'investitore: infatti questo procedimento quantifica, secondo l'approccio di Markowitz, i rendimenti attesi, le volatilità, le correlazioni presenti negli asset disponibili sul mercato che coprono un dato orizzonte temporale di investimento. Tali calcoli conducono generalmente a tracciare gli insiemi efficienti, all'interno dei quali il decisore razionale sceglie, in funzione della sua avversione o propensione al rischio ed eventuali altri fattori caratteristici, le combinazioni soggettivamente ottimali. Mentre la prima fase, data la sua natura quantitativa, ben si presta ad essere modellizzata con tecniche matematico-statistiche tradizionali, ampiamente trattate dalla letteratura specifica (frontiere efficienti, ottimizzazione multi-obiettivo, analisi di regressione multiple, CAPM, SML, alpha/beta analysis), la seconda, di natura maggiormente soggettiva, necessita di una modellizzazione differente. Sempre rimanendo all'interno di un framework quantitativo, si reputa che l'impiego di una tecnica di soft-computing, quale la Fuzzy Logic, possa essere considerata un valido strumento di analisi.

Lo studio si suddivide in due parti: nella prima vengono trattati i principi di funzionamento della Fuzzy Logic, nella seconda viene riportato un case-study sviluppato in Matlab, con riferimento ad un investitore prudente.

\section{La modellizzazione di un sistema secondo i principi della logica sfumata}

La Fuzzy Logic, ossia logica definita "sfumata", rappresenta una tecnica di modellizzazione matematica, impiegabile come strumento di controllo, nonché possibile approccio per il supporto alle decisioni, che negli ultimi anni sta trovando un numero sempre maggiore di applicazioni in contesti reali.

Fondamentalmente, la Fuzzy Logic assume due significati distinti:

- in senso stretto si configura come un sistema logico, inteso come estensione della logica multi-valore;

- in senso più ampio, è quasi sinonimo della teoria dei Fuzzy sets, con riferimento a classi di oggetti con confini sfumati, in cui il concetto di appartenenza assume una questione di grado.

\footnotetext{
${ }^{1}$ BANCA CARIGE - Financial Engineering

${ }^{2}$ Professore ordinario di Analisi Matematica dell'Università di Genova - Dipartimento di Ingegneria Meccanica (DIME)

${ }^{3}$ Dottorando in Computer Science \& Systems Engineering - Università di Genova
} 
Un aspetto estremamente rilevante della Fuzzy Logic consiste sostanzialmente nel fatto che lo sperimentatore tratta variabili linguistiche, ovvero variabili i cui valori sono parole e non numeri; pertanto, molti aspetti della Fuzzy Logic possono essere interpretati come approcci metodologici, atti a processare "parole" piuttosto che "numeri".

Il secondo concetto basilare nella Fuzzy Logic, che gioca un ruolo centrale nella maggior parte delle applicazioni, consiste nell'utilizzo di regole if-then di tipo Fuzzy. Nei sistemi rule-based manca completamente un meccanismo di inferenze logiche che impiegano modelli "antecedenti e conseguenti" a logica sfumata. Vengono pertanto estesi i concetti di implicazione logica, generalizzando i legami che esistono tra una proposizione (antecedente) ed un'altra proposizione (a questa conseguente), in modo da mettere in relazione i rispettivi valori di verità.

La Fuzzy Logic si configura come un valido strumento di supporto alle decisioni, che rientra all'interno del cosiddetto softcomputing: a differenza del tradizionale hard-computing, questa tecnica implementa l'imprecisione del mondo reale, nel senso che si avvale della propria tolleranza nei confronti dell'imprecisione, dell'incertezza e della verità "sfumata", per guadagnare in termini di trattabilità, robustezza e basso costo della soluzione.

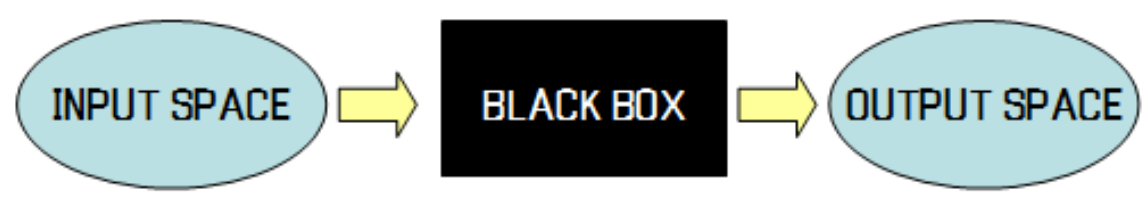

Figura 1: Schema base di mappatura input-output

La Figura 1 mostra il funzionamento del più classico sistema di mappatura di tipo input-output: tra lo spazio di input e quello di output è collocata una black-box, che può contenere diversi strumenti di modellizzazione trattati in letteratura, come sistemi Fuzzy, sistemi lineari, reti neurali, sistemi non lineari, equazioni differenziali. Nel caso in cui la dinamica sia supposta regolata dalla logica Fuzzy, partendo da una certa interpretazione del vettore di input, si perviene all'assegnazione di un determinato valore linguistico alla variabile di output.

In generale, è possibile individuare alcune proprietà, riassunte nel seguito, che mettono in luce i pregi della tecnica descritta:

- semplicità concettuale: i fondamenti matematici basilari sono essenzialmente quelli della logica booleana, che configurano la Fuzzy Logic come un approccio intuitivo;

- flessibilità: dato un qualsiasi sistema, risulta piuttosto semplice implementare nuove funzionalità in via incrementale (mediante la programmazione a blocchi);

- $\quad$ tolleranza nei confronti dei dati imprecisi o di origine incerta;

- facilità di modellizzazione di funzioni non lineari, aventi complessità arbitraria;

- $\quad$ possibilità di associazione e semplice interfaccia alle tradizionali tecniche di controllo;

- essere pienamente descritta dal linguaggio umano naturale in modo "friendly".

\subsection{I fondamenti della Fuzzy Logic}

Utilizzando la Fuzzy Logic, lo sperimentatore ha la possibilità di effettuare una mappatura dello spazio della variabile di input, verso quella di output; il meccanismo primario per poter passare dall'insieme di ingresso del sistema a quello di uscita è costituito dalle cosiddette regole if-then.

L'inferenza Fuzzy è un metodo che interpreta i valori del vettore di input e, sulla base di insiemi di regole definite aprioristicamente dallo sperimentatore (meccanismo if-then), assegna i valori al vettore di output.

Nel prosieguo del paragrafo, vengono delineati i fondamenti teorici e metodologici della Fuzzy Logic.

\section{Fuzzy Sets}

Il primo elemento fondamentale è costituito dai cosiddetti "insiemi Fuzzy", ovvero insiemi i cui confini non sono nettamente delineati, bensì risultano essere "sfumati”. Gli insiemi Fuzzy rappresentano un'estensione della teoria classica degli insiemi, secondo le ipotesi di Lotfi Zadeh, risalenti al 1965.

Tali insiemi possono contenere elementi con un grado parziale di appartenenza (Membership): ogni insieme "sfumato" è caratterizzato da una funzione di grado di appartenenza (Membership Function), che mappa gli elementi di un universo in un intervallo reale continuo $[0 ; 1]$. Il valore nullo sta ad indicare che l'elemento non è sicuramente incluso all'interno del Fuzzy Set, mentre, viceversa, il valore 1 indica una appartenenza certa all'insieme; i valori compresi tra 0 e 1 indicano il grado di appartenenza dell'elemento in oggetto all'insieme "sfumato".

Per un universo $X$ ed una data funzione di appartenenza $\mu_{A}: X \rightarrow[0 ; 1]$, 1'insieme Fuzzy $A$ è definito come:

$$
A=\left\{x, \mu_{A}(x) \mid x \in \mathrm{X}\right\}
$$


dove $\mu_{A}$ è detta funzione di appartenenza di $x$ in $A$, includendo la mappatura di ciascun elemento di $X$ attraverso l'idoneo valore compreso tra 0 e 1 .

Nella letteratura specializzata sono proposte diverse tipologie di funzioni idonee allo scopo quali, ad esempio: funzioni lineari a tratti, gaussiane, sigmoidi, funzioni polinomiali cubiche e quadratiche.

\section{Operatori Matematici}

Una volta introdotta l'inferenza "sfumata", occorre studiare come questa sia connessa alle operazioni logiche. Come accennato, un aspetto peculiare della Fuzzy Logic coincide con un sovra insieme della logica booleana canonica; in particolare, considerando gli estremi del range di variazione, ovvero 1 se completamente vero, oppure 0 se completamente falso, le operazioni logiche standard sono perfettamente rispettate. Ma, poiché nella Fuzzy Logic, la veridicità di qualsiasi affermazione risulta essere funzione del grado di appartenenza, è possibile risolvere l'operazione $A \cap B$, dove gli insiemi sono limitati all'interno del range $[0 ; 1]$, mediante la funzione $\min (A, B)$. Avvalendosi della stessa logica, è possibile sostituire l'operazione di $A \cup B$ con la funzione $\max (A, B)$. Infine, l'operazione $\operatorname{NOT}(A)$ risulta equivalente a $(1-A)$.

La rappresentazione delle tabelle logiche, mutuate dalla logica booleana con gli operatori matematici, è una delle particolarità più interessanti della Fuzzy Logic.

La Figura 2 mostra le tavole di verità con l'esplicazione di quanto sopra esposto:

\begin{tabular}{|c|c|c|}
\hline A & B & $A$ and $B$ \\
\hline 0 & 0 & 0 \\
\hline 0 & 1 & 0 \\
\hline 1 & 0 & 0 \\
\hline 1 & 1 & 1 \\
\hline
\end{tabular}

AND

\begin{tabular}{|c|c|c|}
\hline A & B & $A$ or $B$ \\
\hline 0 & 0 & 0 \\
\hline 0 & 1 & 1 \\
\hline 1 & 0 & 1 \\
\hline 1 & 1 & 1 \\
\hline
\end{tabular}

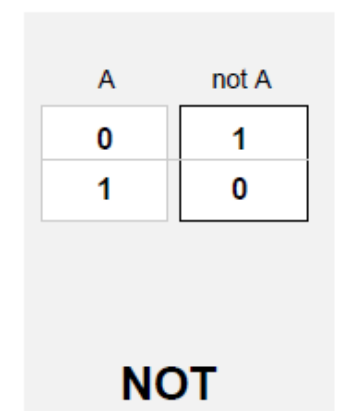

Figura 2: Equivalenza operazioni logiche ed operatori matematici: AND $\rightarrow$ min, OR $\rightarrow$ max e NOT $\rightarrow 1-A$

In linea generale, la Fuzzy Logic estende la logica booleana:

- $\quad$ true $=1$

- false $=0$;

- sono incluse comunque tutte le "sfumature" comprese all'interno degli estremi 0 e 1 del range.

\section{Membership Functions}

Una Membership Function (MF) è una curva che fondamentalmente definisce come ciascun punto nello spazio di input viene mappato con un determinato grado di membership, compreso tra 0,1 e il grado con cui un determinato oggetto appartiene ad un Fuzzy Set è identificato pienamente dal valore di appartenenza.
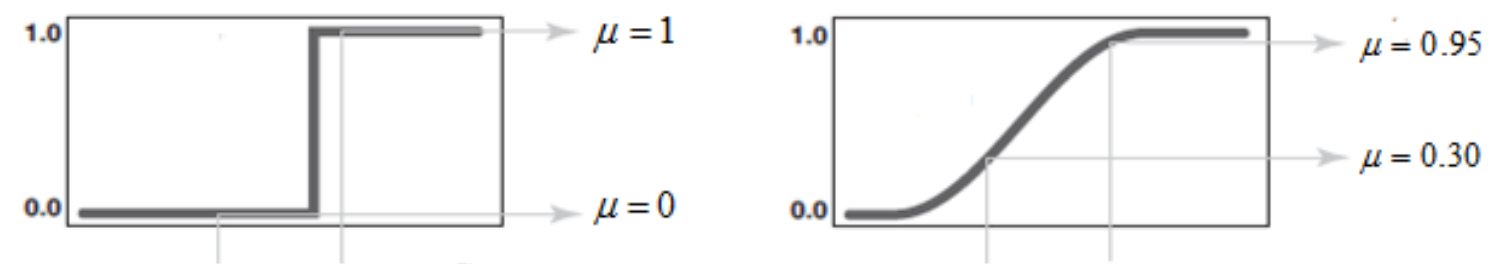

Figura 3: Rappresentazione della classica funzione a gradino (logica binaria) VS sigmoide (logica Fuzzy)

\section{If-then Rules}

All'interno della Logica Fuzzy, i Fuzzy sets e gli operatori matematici descritti nelle precedenti sezioni, costituiscono metaforicamente il soggetto ed il verbo della logica "sfumata". In questa sezione viene introdotto il concetto di regole if-then, implementate nella metodologia per formulare le istruzioni condizionali, che compongono tutta la tecnica.

Una semplice regola assume questa forma: IF $x$ is $A$, THEN $y$ is $B$

dove $A$ e $B$ sono i valori linguistici associati alle variabili linguistiche $x$ e $y$, sui range $X$ e $Y$. 
In linea di principio, una regola if-then riceve in input il valore linguistico della variabile di ingresso, ovvero l'antecedente viene interpretato, ritornando un valore compreso tra 0 e 1 . A tale valore corrisponde, nell'insieme di output $B$, un'assegnazione $y$ (definita fase di implicazione).

Il risultato, espresso in forma codificata, verrà successivamente defuzzificato, restituendo in tal modo un valore facilmente interpretabile.

Il processo di interpretazione di una regola IF-THEN è tipicamente costituito da due step distinti:

1. valutazione dell'input: processo che consiste nel fuzzificare l'input (Fuzzification) e, se necessario, applicare operatori Fuzzy (nel caso siano presenti più input).

2. applicazione del risultato al conseguente: fase di implicazione.

Un aspetto che contraddistingue la logica "sfumata" da quella binaria è che, mentre in quest'ultima, se la premessa è vera, allora anche la conclusione è tale, nella Fuzzy Logic si verifica che "se la premessa è vera con un determinato grado di appartenenza, allora la conseguenza è vera con lo stesso grado".

Affinchè la metodologia sia efficiente, occorre che il numero di regole utilizzate per descrivere il sistema sia superiore o uguale a due. Gli insiemi Fuzzy di output di ciascuna regola devono poi essere aggregati all'interno di un insieme Fuzzy complessivo di output, che a sua volta viene defuzzificato (Defuzzification), ovvero ricondotto ad un valore numerico.

\subsection{Il processo d'inferenza Fuzzy}

Per processo di inferenza Fuzzy si intende il processo di mappatura da un dato spazio di input verso un opportuno spazio di output, mediante l'utilizzo della tecnica "sfumata". Tale processo si configura come un valido supporto per lo sperimentatore, che deve prendere decisioni. In generale, esso include tutti gli elementi spiegati precedentemente, ovvero: insiemi Fuzzy, operatori matematici, Membership Functions e regole if-then. La figura sottostante mostra la struttura di un sistema Fuzzy, costituito rispettivamente da $m$ input, $n$ output e $k$ regole.

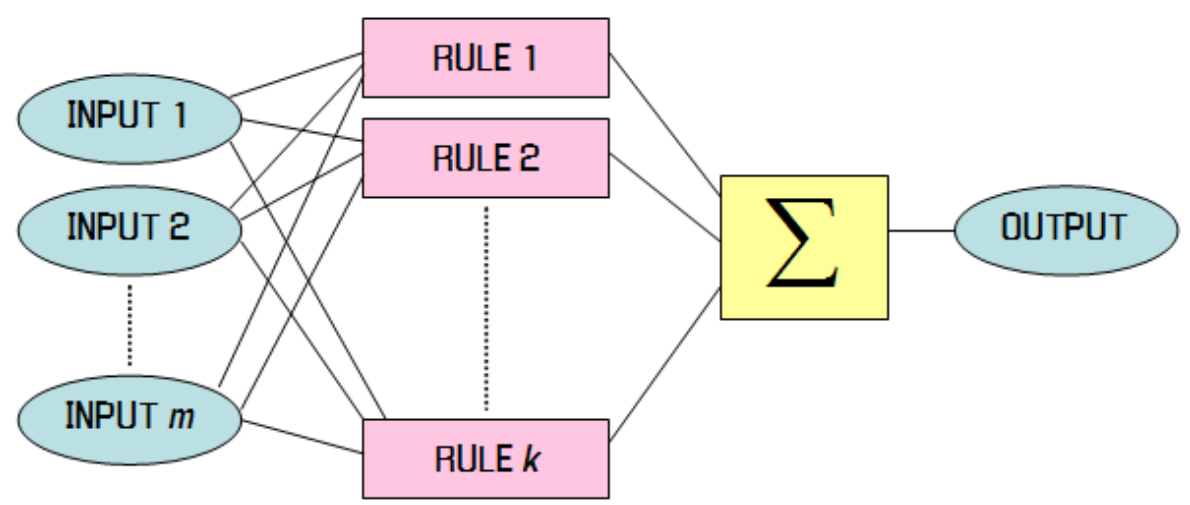

Figura 4: Rappresentazione generale del processo d'inferenza Fuzzy

Gli input del sistema sono valori che variano tipicamente all'interno di un range individuato a priori dallo sperimentatore.

Le regole sono valutate in parallelo, seguendo la logica del ragionamento Fuzzy: questa è un'ulteriore peculiarità del modello che nega l'esistenza di una regola dominante, potendo così passare senza "punti di rottura" da regioni dello spazio in cui il comportamento del sistema è spiegato maggiormente da una regola o da un'altra. I risultati delle regole vengono infine combinati e defuzzificati.

L'output del modello è pertanto espresso in modo non codificato.

Nel prosieguo del paragrafo vengono mostrati i cinque passi fondamentali che caratterizzano il processo di inferenza Fuzzy, secondo il criterio di implementazione nel Fuzzy Logic Toolbox di Matlab.

\section{A. Fuzzificazione delle variabili di input (Fuzzification)}

Il primo passo, che corrisponde alla fase di design del modello, prende in considerazione tutte le variabili linguistiche di input e, per ciascuna, ne associa i relativi valori linguistici; essi sono caratterizzati da apposite funzioni di appartenenza, scelte a discrezione dallo sperimentatore.

In sostanza, si inseriscono variabili, che assumono valori all'interno di un range definito dal modellizzatore e, mediante la fuzzificazione, si determina il grado di appartenenza compreso tra 0 e 1.

Prima di procedere allo step successivo, occorre che tutti gli input siano fuzzificati a seconda dei peculiari set linguistici.

\section{B. Applicazione degli operatori Fuzzy}


A questo punto, per ogni regola che è stata definita aprioristicamente, lo sperimentatore conosce il grado con cui ogni parte dell'antecedente è soddisfatta. Nel caso l'antecedente di una regola sia costituita da più parti, è necessario applicare gli operatori Fuzzy.

\section{Applicazione del metodo d'implicazione}

La terza fase del processo di inferenza prende in input un numero dato dall'antecedente e restituisce in output un insieme Fuzzy.

Prima di applicare il metodo di implicazione, occorre assegnare a ciascuna regola il proprio peso: generalmente, tutte le regole sono pesate in maniera uguale, assumendo un peso pari a 1; ciò consente un bilanciamento fra le regole, evitando l'influenza preponderante di ciascuna rispetto alle altre.

Se l'operatore di implicazione scelto è il min (default) e se le regole hanno tutte peso uguale, il profilo del conseguente viene troncato alla percentuale calcolata dall'antecedente. Se i pesi associati alle singole regole sono diversi, il profilo del conseguente sarà variegato, in funzione dell'importanza data alle singole rules.

\section{Aggregazione di tutti gli output}

In questa fase, gli output di ciascuna regola devono essere combinati in modo da assumere una decisione. L'aggregazione consiste nell'opportuno processo di combinazione dei vari output di ogni regola. Da sottolineare che l'output di ogni singola regola corrisponde ad un Fuzzy set e questo step è strettamente necessario per andare ad aggregare più insiemi "sfumati", ottenendone uno complessivo.

L'aggregazione è commutativa, ossia l'ordine con cui si considerano le uscite di ogni singola regola è totalmente invariante.

L'implementazione dell'aggregazione può essere realizzata con il metodo del max (default), min o del sum.

\section{E. Defuzzificazione (Defuzzification)}

L'ultimo step del processo di inferenza Fuzzy è definito Defuzzificazione: prendendo in ingresso il Fuzzy set aggregato, ottenuto al passo precedente, restituisce in output un numero singolo che verrà utilizzato dallo sperimentatore a conclusione del metodo a supporto della propria decisione. Tra le tecniche di defuzzificazione, una delle più comuni è il calcolo del centroide, ovvero del baricentro dell'area sottostante la curva di output. Altre valide tecniche impiegate sono il bisettore, la media del massimo, il più grande dei massimi oppure il più piccolo dei massimi.

\section{Applicazione della metodologia Fuzzy alle decisioni di investimento}

Dopo aver trattato la teoria alla base del funzionamento della modellizzazione in ottica sfumata, viene presentata un'applicazione, realizzata in ambiente Matlab, che, avvalendosi del Fuzzy Logic Toolbox, fornisce un valido supporto alle decisioni di investimento. In particolare, si ha l'intenzione di focalizzare la trattazione sulla fase di scelta dell'asset propria e caratteristica del singolo investitore: come riportato nell'introduzione, nella teoria moderna di gestione del portafoglio, solo la prima parte del processo di individuazione degli asset è di natura oggettiva, descrivibile con metodologie statistiche tradizionali ed indipendente dalle inclinazioni comportamentali dell'investitore (si pensi ad esempio al concetto di avversione al rischio).

Pertanto, facendo riferimento alle metodologie classiche presenti nella letteratura tecnica specifica (Capital Asset Pricing Model (CAPM), Markowitz model, Multi-index Regressive model, alpha/beta analysis), si presuppone di essere in grado di effettuare un'analisi matematico-finanziaria completa del portafoglio in esame.

In particolare, si presume di conoscere e poter quantificare, tramite queste tecniche, il rendimento atteso ed il rischio (quantificabile in termini di deviazione standard, di beta e/o delle correlazioni) associato ad ogni singolo titolo presente nel portafoglio detenuto dall'investitore.

Una volta calcolate queste grandezze, si può interpretare, esaminando le scelte effettuate in passato, il suo profilo comportamentale in funzione del rischio che ha deciso di tollerare e del rendimento a cui normalmente tende ad ambire. Le soglie secondo cui un rendimento passa da "essere interessante" ad "essere trascurabile" o, dualmente, un rischio passa da "essere sopportabile" ad "insostenibile", non sono ovviamente ben marcate e quindi si prestano ad essere le variabili linguistiche da modellizzare in un'ottica sfumata.

Al fine di impiegare il modello in un'ottica generale, è bene utilizzare la notazione standard delle variabili nel campione esaminato sia nell'insieme dei rendimenti sia in quello del rischio. In tal modo il livello zero esprime il rischio che l'investitore è disposto ad accettare in media al fine di ottenere quel rendimento che normalmente lo soddisfa. Ovviamente la corrispondenza del livello zero al suo valore non standardizzato è strettamente connessa al profilo di avversione al rischio caratteristica di ciascun investitore; pertanto si comprende l'esigenza di ricorrere alla standardizzazione degli input, in modo tale che la procedura abbia una validità generale e non sia fortemente tipizzata.

Nella modellizzazione riportata nell'esempio le variabili linguistiche di ingresso da descrivere secondo appropriate Membership Functions sono il "Rendimento" ed il "Rischio", che possono essere rappresentate dal set di valori linguistici "Basso", "Medio" ed "Alto". Le funzioni di appartenenza impiegate nel modello sono di natura gaussiana e sono riportate nella Figure 5 e 6. La variabile linguistica di output è "Investimento", che può essere descritta dalle parole "Pessimo", "Non Consigliabile", "Allineato", "Consigliabile" e "Ottimo" e le cui funzioni normali di appartenenza sono illustrate nella Figura 7. 
L'obiettivo che ci si pone è quello di esprimere un giudizio sull'investimento in esame confrontandolo con i titoli già detenuti nel portafoglio dell'investitore: paragonando il suo rendimento atteso con quello attuale di portafoglio e confrontando il suo livello di rischio con quello attualmente presente nel portafoglio, il fine del programma è quello di definire l'investimento esaminato sia in termini linguistici (ad es. consigliabile/non consigliabile) sia in termini numerici, ovvero fornire un rank da 0 a 10 sulla bontà di tale scelta.

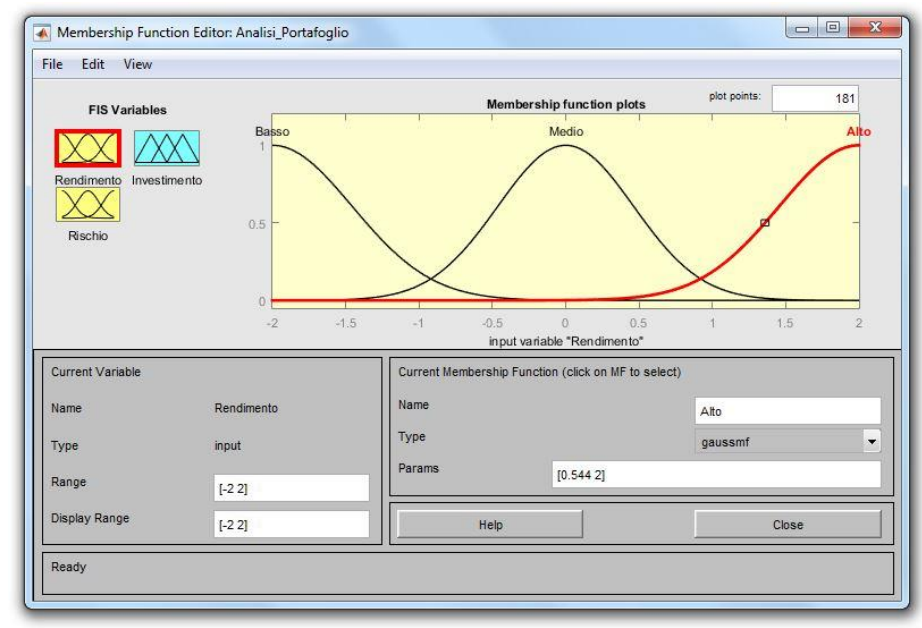

Figura 5: Membership Function della variabile linguistica "Rendimento"

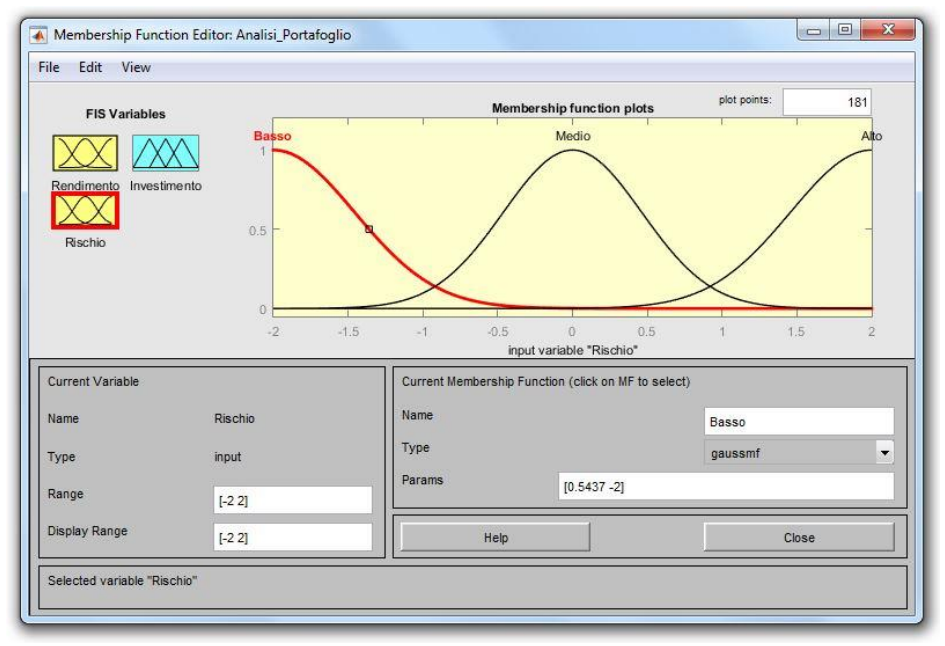

Figura 6: Membership Function della variabile linguistica "Rischio"

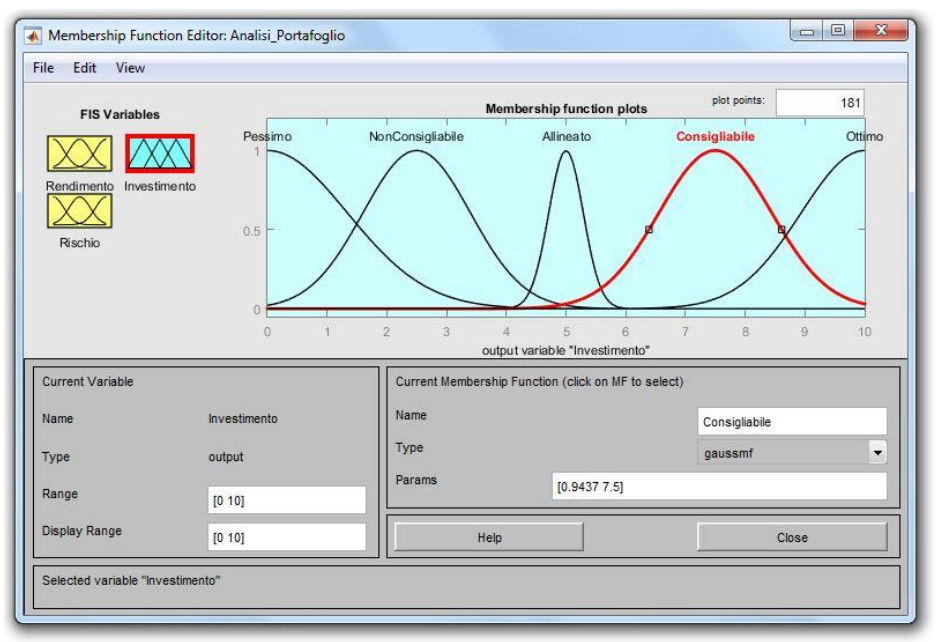

Figura 7: Membership Function della variabile linguistica "Investimento" 
Gli input e l'output sono legati da un insieme di regole logiche, che rispecchiano, in questo caso, un profilo di rischio di un investitore prudente, in accordo con quanto riportato nella Tabella 1.

\begin{tabular}{|c|c|c|c|}
\hline OUTPUT MAPPING & Rischio BASSO & Rischio MEDIO & Rischio ALTO \\
\hline Rendimento BASSO & Non Consigliabile & Non Consigliabile & Pessimo \\
\hline Rendimento MEDIO & Consigliabile & Allineato & Non Consigliabile \\
\hline Rendimento ALTO & Ottimo & Consigliabile & Non Consigliabile \\
\hline
\end{tabular}

Tabella 1: Mappatura delle due variabili linguistiche di Input (Rischio-Rendimento) con l'output (Investimento)

Questa mappatura viene elaborata dal software fornendo un insieme di regole (Rules) che interpretano la matrice sopra riportata. Le logiche adottate sono implementate nel modo illustrato nella Figura 8.

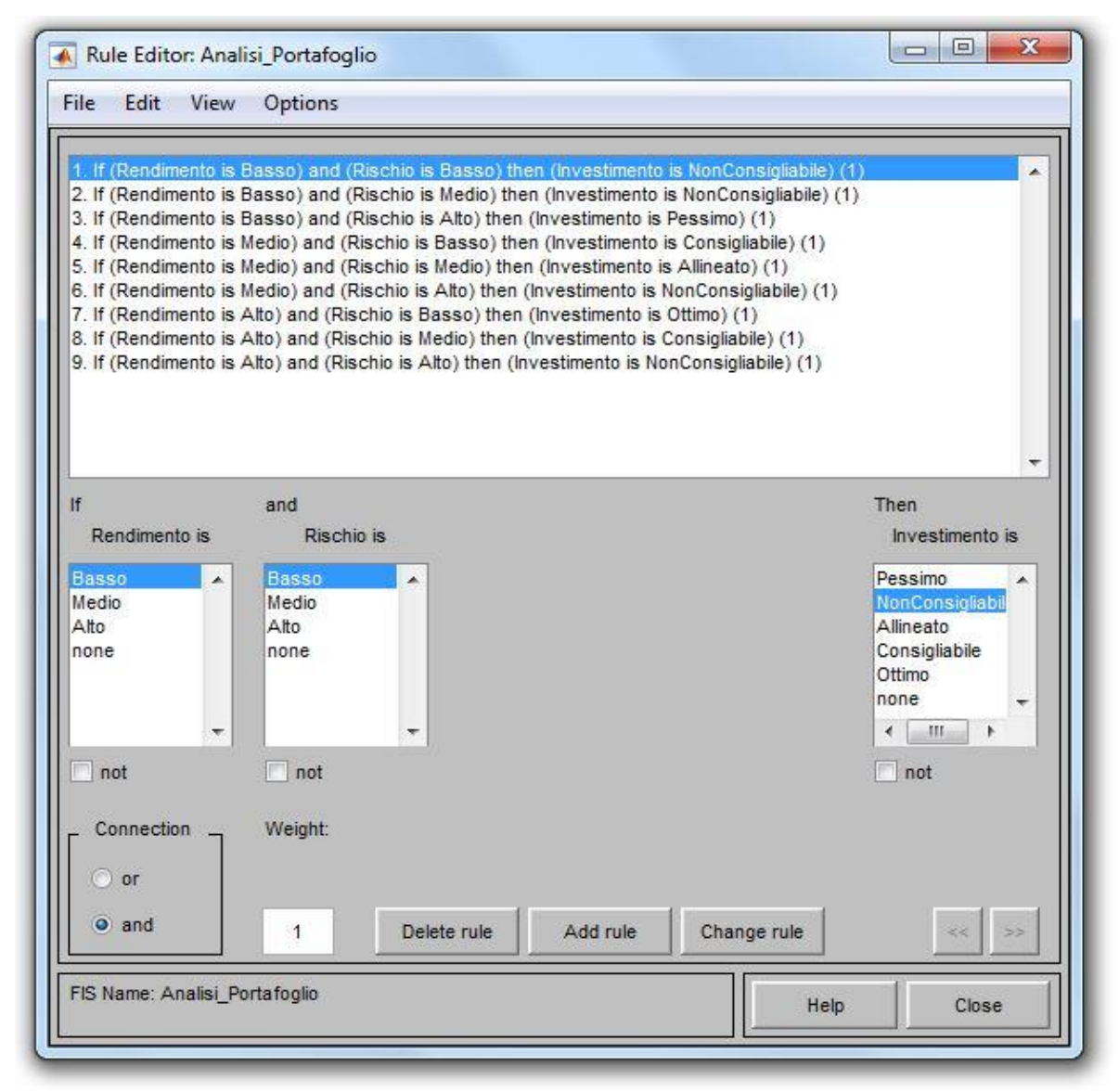

Figura 8: Rule Editor - Specifica delle regole

Definite le variabili linguistiche di ingresso e di uscita del modello, le relative funzioni di appartenenza e le regole che esprimono le relazioni esistenti tra loro, diviene ora necessario specificare i parametri tecnici caratteristici per la risoluzione del problema.

Nella Figura 9 viene presentato il Designer per il sistema di Mamdani in esame: l'interfaccia grafica consente di definire il tipo di operazioni da effettuare per i metodi AND ( $\min$ ) ed OR ( $\max$ ), per le funzioni di implicazione ( min ), aggregazione ( $\max$ ) e defuzzificazione (centro di massa).

Gli operatori così impostati sono quelli normalmente consigliati dalla letteratura scientifica. 


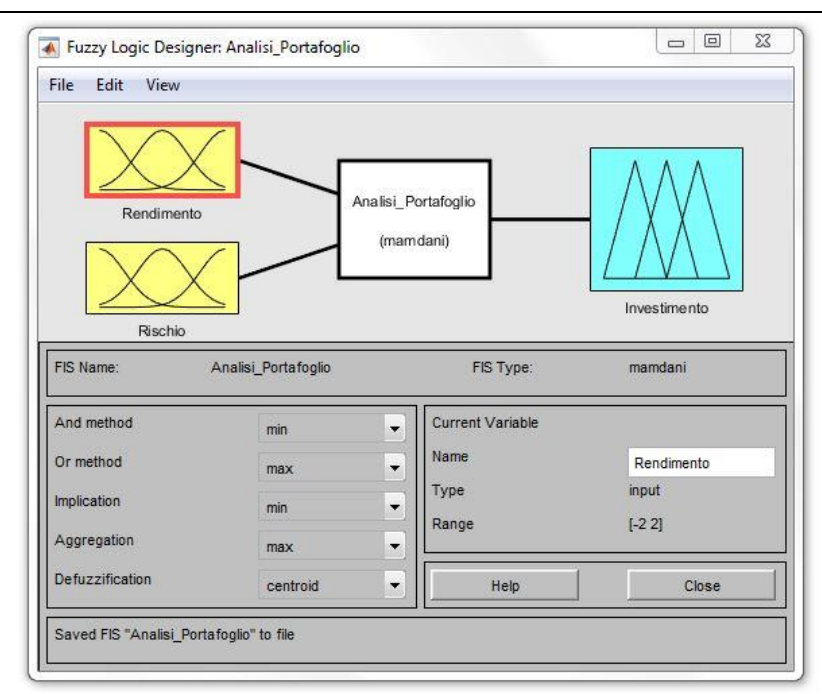

Figura 9: Fuzzy Logic Designer

Giunti a questo livello, il modello è interamente definito e pronto per l'utilizzo. Nel prosieguo del paragrafo sono mostrati tre esempi intuitivi, in modo da rendere più agevole la comprensione della metodologia agli addetti ai lavori. Per ciascuno di questi casi e per ognuna delle nove rules utilizzate, viene illustrato graficamente il procedimento di implication, aggregation $\mathrm{e}$ defuzzification.

\section{Caso A: Investimento completamente "in linea" con il portafoglio attuale}

Il nuovo investimento preso in considerazione ha i medesimi parametri di rendimento e rischio di quello ad oggi posseduto. Pertanto il suo rendimento e il relativo parametro di rischio, espresso nelle variabili standardizzate, è zero. In Figura 10 è riportata l'intera analisi di questa casistica: in corrispondenza delle nove regole logiche riportate sulle righe, si evidenzia l'impatto del valore nullo del rendimento e del rischio sulle rispettive funzioni d'appartenenza (prime due colonne). La terza colonna, per ognuna delle nove regole, visualizza il risultato degli operatori logici (AND/OR) e delle relative implicazioni sulla funzione di appartenenza della variabile di output (implication). Il profilo riportato nella decima riga della terza colonna rappresenta l'aggregazione dei nove profili derivanti dalle implicazioni di riga (aggregation). La linea rossa rappresenta il centro di massa della figura (defuzzification) e consente di esprimere quantitativamente un indice di bontà dell'investimento pari a 5 su 10. Il rank associato all'investimento, che costituisce l'output ultimo del programma, è riportato sinteticamente come intestazione della terza colonna. Il giudizio dell'investimento, secondo il modello proposto, è pertanto "allineato" ed il voto è di poco non sufficiente in quanto non apporta comunque miglioramenti alla configurazione attuale.

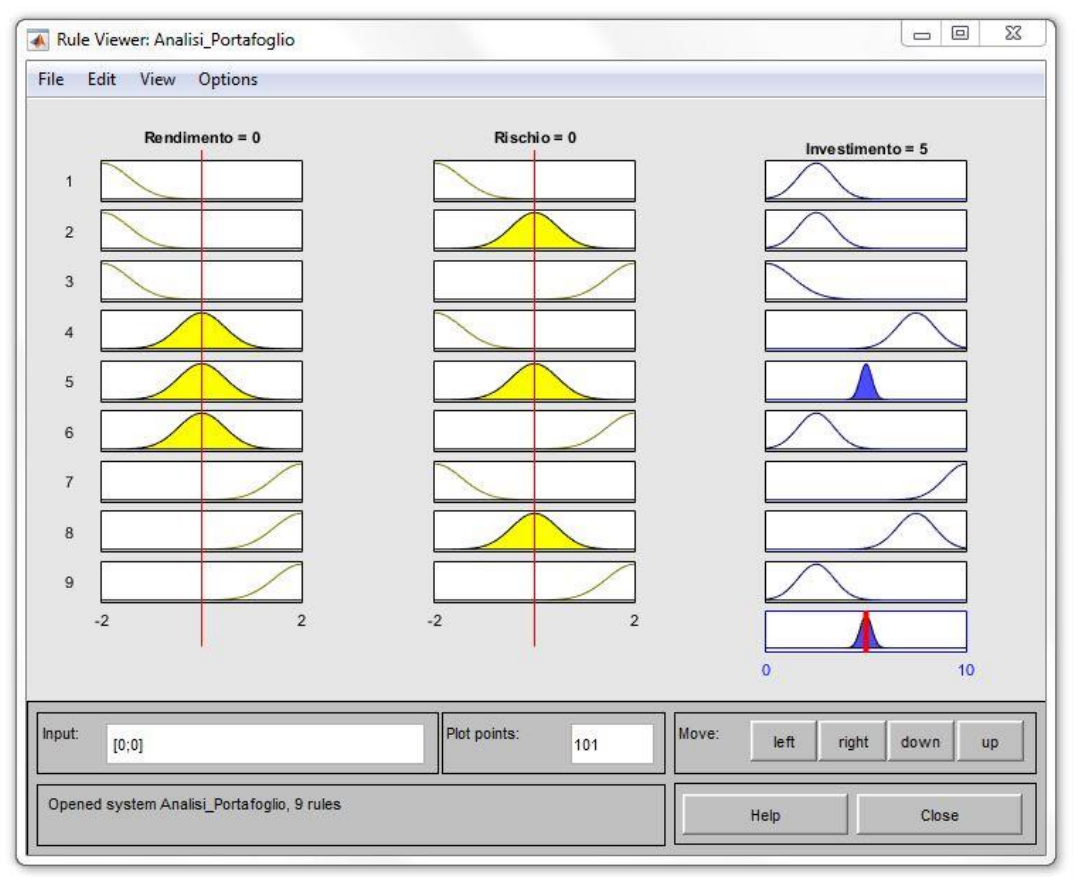

Figura 10: Analisi grafica in logica sfumata di un investimento in linea alle caratteristiche finanziarie del portafoglio attuale 

portafoglio attuale

Il nuovo investimento ha un rendimento, espresso nelle variabili standardizzate, pari a +0.5 e un fattore di rischio pari a +1.5 . Come si evince dalla figura sottostante, il rank dell'investimento, secondo un profilo prudente di avversione al rischio, è pari a 2.58 e risulta pertanto non consigliabile.

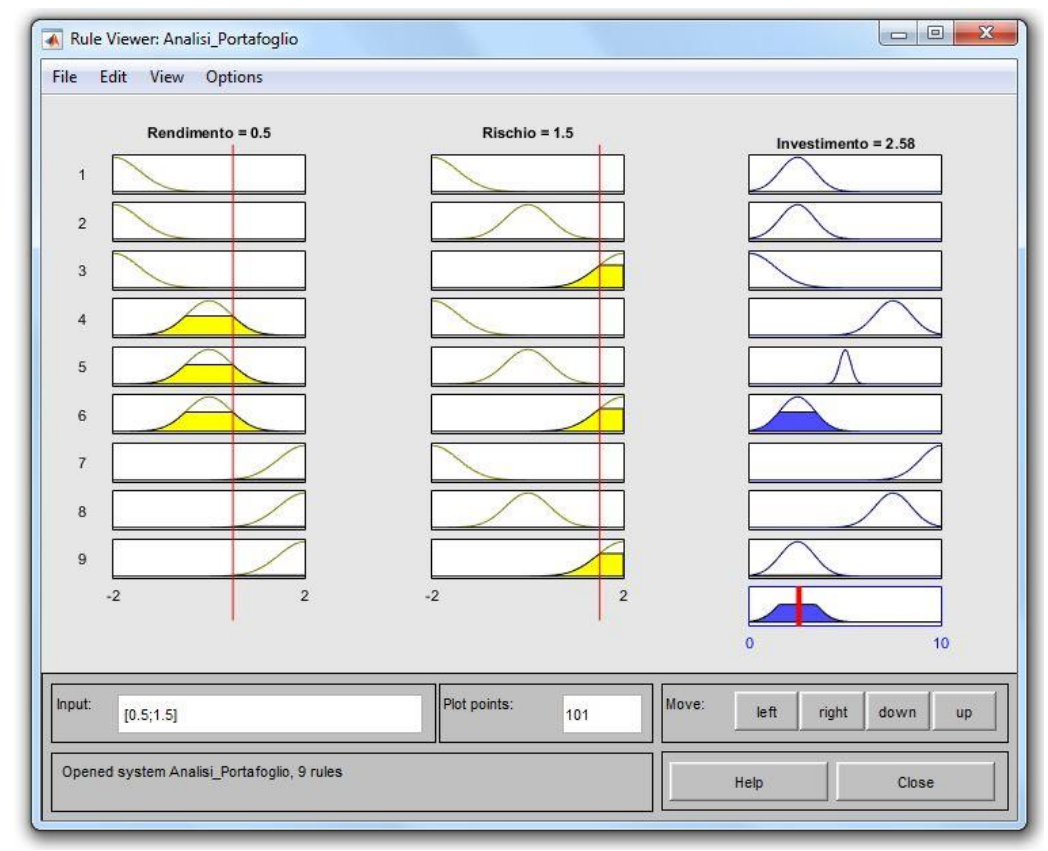

Figura 11: Analisi grafica in logica sfumata di un investimento che a fronte di un incremento moderato del rendimento richiede un'esposizione significativa al rischio

Caso C: Investimento che a fronte di un incremento contenuto del rischio aumenta in modo sensibile il rendimento atteso del portafoglio attuale

Il nuovo investimento ha un rendimento, espresso nelle variabili standardizzate, pari a +1.5 e un fattore di rischio pari a +0.5 . Come si evince dalla Figura 12 il rank dell'investimento, secondo un profilo prudente di avversione al rischio, è pari a 7.2 e dunque risulta consigliabile.

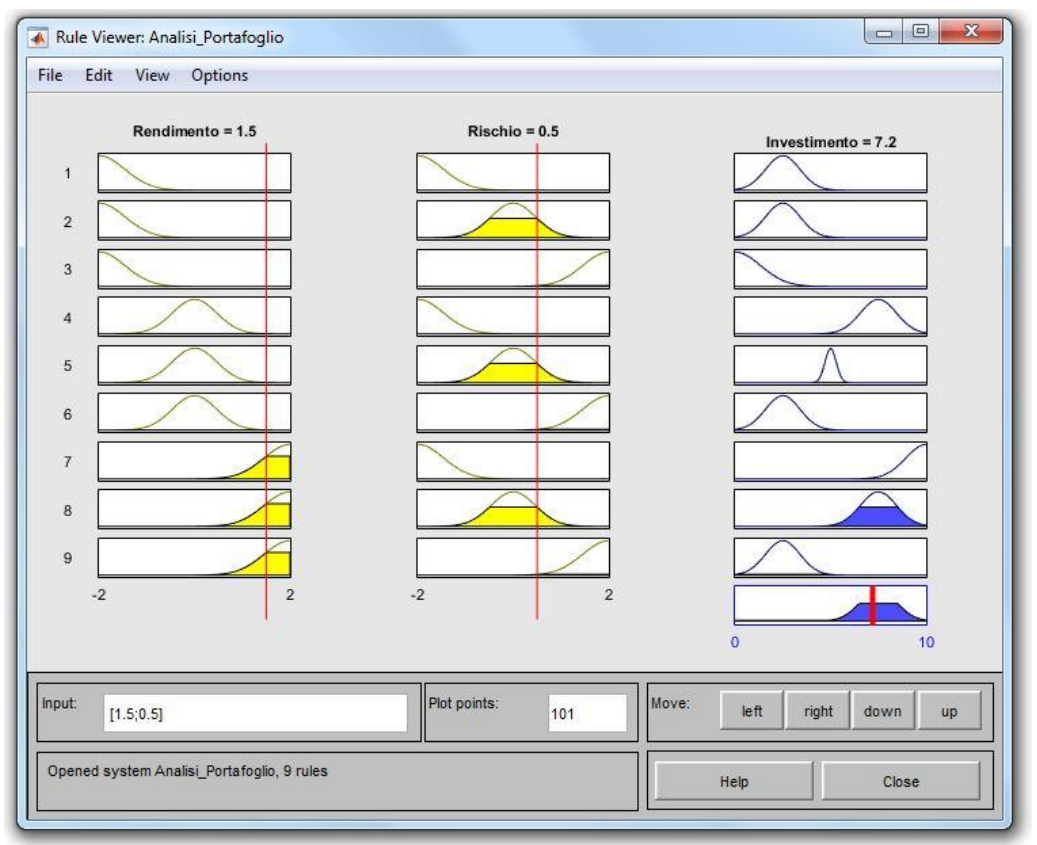

Figura 12: Analisi grafica in logica sfumata di un investimento che a fronte di un incremento moderato del rischio si ottiene un rendimento significativamente superiore a quello del portafoglio attuale 
Variando i valori standardizzati associati alle due variabili d'ingresso, Rendimento e Rischio, nel range $[-2,+2]$ si è in grado di ottenere l'intera superficie di risposta del sistema Fuzzy in esame. La grigliatura dei fattori, riportata nella Figura 13, mostra tutte le possibili casistiche per un investitore prudente che segue le regole if-then riportate nella Tabella 1.

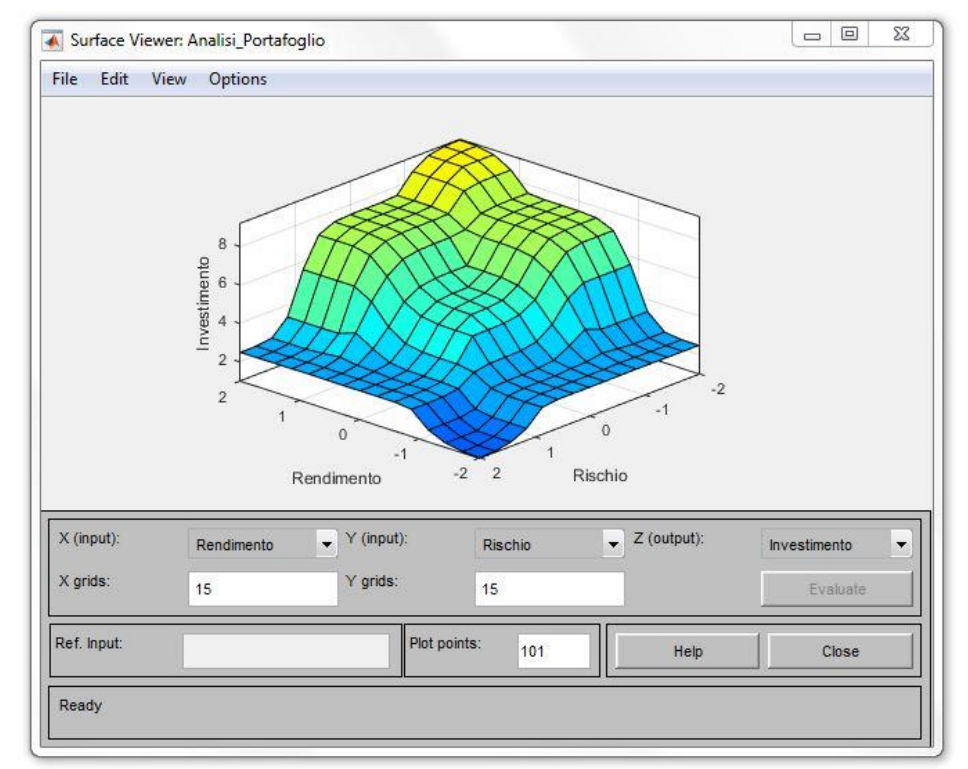

Figura 13: Surface Viewer - La superficie mostra per ogni livello assunto dai fattori di Rischio e Rendimento il corrispondente rank per l'investimento in esame secondo un profilo prudente di investitore

\section{Conclusioni}

L'articolo ha presentato in che modo una metodologia di soft-computing, quale la Fuzzy Logic, possa essere integrata nella moderna teoria di Markovitz per la gestione ottimale di un portafoglio di asset. Tale tipo di algoritmo, partendo dalle scelte di investimento pregresse di un investitore razionale e dalla sua percezione del rischio, fornisce, grazie alla rielaborazione in ottica sfumata di tali informazioni soggettive, un criterio per la valutazione di una nuova proposta di investimento. Si reputa interessante per il proseguo dello studio applicare i concetti della Fuzzy Logic ad altri campi della finanza e della economia, soprattutto quando alla quantificazione esatta di una variabile di modello, si preferisce una caratterizzazione mediante descrizione linguistica della stessa.

\section{Bibliografia}

[1] G. Stojic - "Using Fuzzy Logic for evaluating the level of countries' (regions') Economic Development" Panoeconomicus, 2012

[2] T. Korol - "Fuzzy Logic - Emerging Technologies and Applications: Fuzzy Logic in Financial Management" - InTech Pubblication, 2012

[3] G. Bojadziev, M. Bojadziev - "Fuzzy Logic for Business, Finance and Management" - Advances in Fuzzy Systems: Applications and Theory, Vol. 23, 2007

[4] J. Harris - "Fuzzy Logic Applications in Engineering Science", Springer series in "Microprocessor based and intelligent systems engineering”, Vol. 29,2006

[5] The Mathworks - "Fuzzy Logic Toolbox" - User's Guide, 2015

[6] A. M. Ribeiro, L. B. Neto, P. H. G. Coelho, J. C. Soares de Mello, L. A. Meza - "Using Fuzzy Logic for pricing" - ICEIS 2005 - Artificial Intelligence and Decision Support Systems, 2005

[7] A. M. Gil Lafuente - "Fuzzy Logic in Financial Analysis" - Springer series in "Fuzziness and Soft Computing", Vol. 175, 2005

[8] Dispense del Master Internazionale CIIA® (Certified International Investiment Analyst), Sezione: "Portfolio Management", 2015

[9] L. A. Zadeh - "Fuzzy Sets" - Information and Control, Vol 8, 1965

[10] L. A. Zadeh - "Fuzzy Algorithms" - Information and Control, Vol 5, 1968

[11] L. A. Zadeh - "The concept of a linguistic variable and its application to approximate reasoning", Information Sciences, 8,1975

[12] L. A. Zadeh - "A meaning representation language for natural languages", International Journal of Man-Machine Studies, 1978

[13] J. Harris - "An introduction to Fuzzy Logic Applications", Kluwer Academics Publishers, Dordrecht, 2000

[14] H. J. Zimmermann - "Fuzzy Set Theory and its Applications", Kluwer-Nijho Publishing, Boston (1984) 


\section{Newsletter AIFIRM - Risk Management Magazine}

Anno 11, n 3-4 Luglio - Dicembre 2016

Direttore Responsabile

Maurizio Vallino

\section{Condirettore}

Corrado Meglio

\section{Consiglio Scientifico}

Simona Cosma

Paola Ferretti

Giampaolo Gabbi

Andrea Giacomelli

Pier Giuseppe Giribone

Cristiana Schena

Giuseppe Torluccio

Enzo Scannella

Comitato di redazione

Emanuele Diquattro

Fausto Galmarini

Rossano Giuppa

Aldo Letizia

Paolo Palliola

Enzo Rocca

Fabio Salis

Vignettista: Silvano Gaggero

Proprietà, Redazione e Segreteria:

Associazione Italiana Financial Industry Risk Managers (AIFIRM), Via Sile 18, 20139 Milano

Registrazione del Tribunale di Milano nº 629 del 10/9/2004

E-mail: segreteria@ aifirm.it;

Tel. 3896946315

Lunedì - Venerdì h.15-17

Stampa: Algraphy S.n.c. - Passo Ponte Carrega 62-62r

16141 Genova

Le opinioni espresse negli articoli impegnano unicamente la responsabilità dei rispettivi autori

SPEDIZIONE IN ABBONAMENTO POSTALE AI SOCI AIFIRM RESIDENTI IN ITALIA, IN REGOLA CON L'ISCRIZIONE 


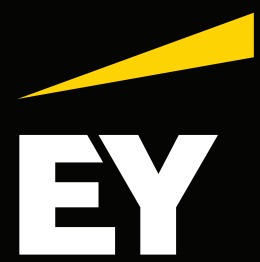

Building a better working world

e

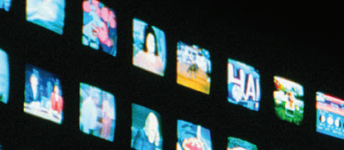

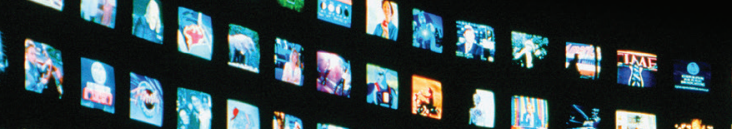

int

AR

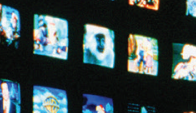

$4 \operatorname{lin}(x)$

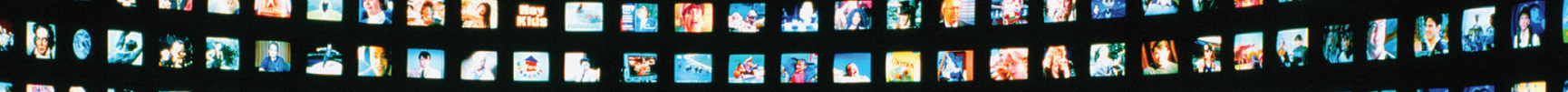
3 29 店 19.

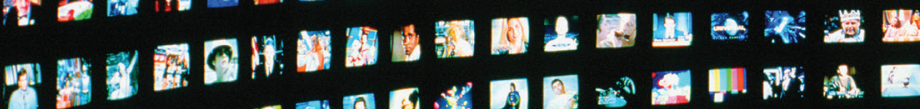

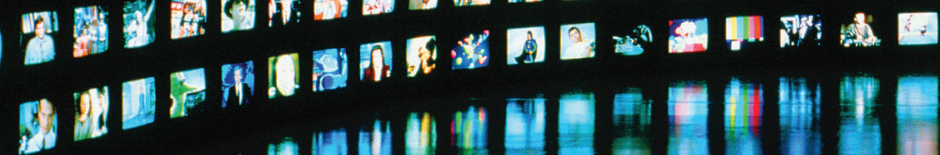

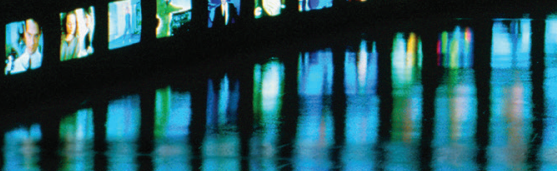

$\infty$

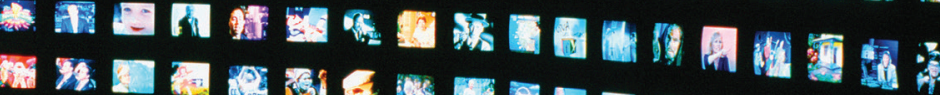

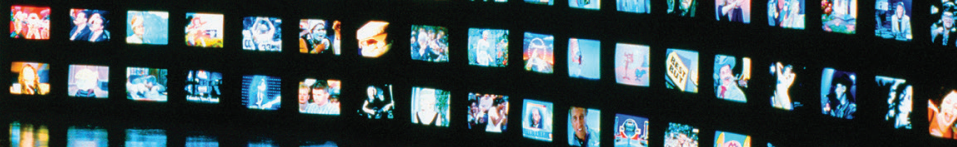
$=1$

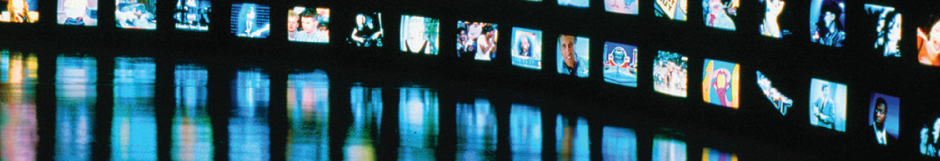

\section{Is security the price to pay for innovation?}

EY's global financial services team can help you transform and improve your cyber security capabilities. ey.com/financial \#BetterQuestions

The better the question. The better the answer. The better the world works. 